\title{
On the Usefulness or Lack Thereof of Optimality Criteria for Structural Change Tests*
}

\author{
Pierre Perron ${ }^{\dagger}$ \\ Boston University
}

\author{
Yohei Yamamoto ${ }^{\ddagger}$ \\ Hitotsubashi University
}

Revised: June 2, 2013.

\begin{abstract}
Elliott and Müller (2006) considered the problem of testing for general types of parameter variations, including infrequent breaks. They developed a framework that yields optimal tests, in the sense that they nearly attain some local Gaussian power envelop. The main ingredient in their setup is that the variance of the process generating the changes in the parameters must go to zero at a fast rate. They recommended the so-called $q \hat{L} L$ test, a partial sums type test based on the residuals obtained from the restricted model. We show that for breaks that are very small, its power is indeed higher than other tests, including the popular sup-Wald test. However, the differences are very minor. When the magnitude of change is moderate to large, the power of the test is very low in the context of a regression with lagged dependent variables or when a correction is applied to account for serial correlation in the errors. In many cases, the power goes to zero as the magnitude of change increases. The power of the sup-Wald test does not show this non-monotonicity and its power is far superior to the $q \hat{L} L$ test when the break is not very small. We claim that the optimality of the $q \hat{L} L$ test does not come from the properties of the test statistics but the criterion adopted, which is not useful to analyze structural change tests. Instead, we use fixed-break size asymptotic approximations to assess the relative efficiency or power of the two tests. When doing so, it is shown that the sup-Wald test indeed dominates the $q \hat{L} L$ test and, in many cases, the latter has zero relative asymptotic efficiency.
\end{abstract}

\section{JEL Classification Number: C22.}

Keywords: structural change, local asymptotics, hypothesis testing, parameter variations.

\footnotetext{
*Perron acknowledges financial support for this work from the National Science Foundation under Grant SES-0649350. We are grateful to Adam McCloskey and a referee for useful comments on a previous draft.

${ }^{\dagger}$ Department of Economics, Boston University, 270 Bay State Rd., Boston, MA, 02215 (perron@bu.edu).

${ }^{\ddagger}$ Department of Economics, Hitotsubashi University, 2-1, Naka, Kunitachi, Tokyo, 186-8601, Japan (yohei.yamamoto@econ.hit-u.ac.jp).
} 


\section{Introduction}

The problem of testing for structural changes has been an active area of theoretical and applied research for over 50 years (see Perron, 2006). In the last fifteen years or so, substantial advances have been made to cover models at a level of generality that allows a host of interesting practical applications in the context of unknown change points. These include models with general stationary regressors and errors that can exhibit temporal dependence and heteroskedasticity. In this respect, Andrews (1993) considered the limit distribution of the so-called Sup-type tests, which are based on the maximal value of some statistic over possible break dates within a pre-specified set that excludes some proportion of the data near the beginning or the end of the sample. Bai and Perron (1998) generalized this approach to the case of multiple structural changes in the context of the linear model. Andrews and Ploberger (1994) developed an asymptotic analysis based on a local asymptotic

framework, whereby the parameters under the alternative hypothesis are made local to the null value. These tests are optimal in the sense that they maximize a weighted average of the local asymptotic power envelop. More recently, Elliott and Müller (2006) (henceforth EM) considered the problem of testing for general types of parameter variations, including infrequent breaks. They developed a framework that yields optimal tests, in the sense that they (nearly) attain some local Gaussian power envelop. The main ingredient in their setup is that the variance of the process generating the changes in parameters must go to zero at a fast rate. This allows them to show that all small sample efficient tests for parameter instability are asymptotically equivalent. Hence, one does not need to specify the exact nature of the alternative hypothesis to have a test that is efficient and optimal against any such particular alternative hypothesis. The dramatic implication is that one can choose any such small sample efficient test and it will be so-called optimal when some conditions are satisfied, the most important being that the breaks are local to zero as the sample size increases. Accordingly, they recommend the $q \hat{L} L$ test, an extension of the most powerful invariant test in a Gaussian unobserved component model as analyzed by Franzini and Harvey (1983) and Shively (1988). They show the validity of their test under general conditions including dynamic models and models with serially correlated errors provided it is "robustified" to account for this correlation. In the terminology of Perron (2006), the $q \hat{L} L$ test is a partial sums type test, as initially proposed by Gardner (1969). It is based on the residuals obtained from the model restricted to satisfy the null hypothesis of no change.

This result appears quite impressive as one can make a very wide class of tests to be 
"optimal". Moreover, the literature that uses the $q \hat{L} L$ indeed labels it as an "optimal" test for changes in parameters. Our claim in this paper is that the framework EM adopt to yield an optimality criterion is rather useless, in the sense that it labels as optimal a test with very bad finite sample properties when confronted with practically relevant types of breaks. The main problem is the adoption of a framework in which the breaks are local to zero. Such a setup used to derive optimality criteria has already been shown to be inadequate in other contexts. Deng and Perron (2008) considered the CUSUM and CUSUM of squares tests (Brown, Durbin and Evans, 1975) and showed that the local asymptotic theoretical framework can give a very unreliable guide to the finite power function, especially when dynamics are involved with the errors and a correction is applied to account for this. Kim and Perron (2009) also criticized the local asymptotic framework used by Andrews and Ploberger (1994). They compared the asymptotic relative efficiency of the Exp, Mean, and Sup functionals of the Wald, LM and LR tests for structural change using the criterion of the relative approximate Bahadur slopes of the tests. They showed that tests based on the Mean functional are inferior to those based on the Sup and Exp when the same base statistic is used. Also, for a given functional, the Wald-based test dominates the LR-based test, which dominates the LM-based test. They also compared tests based on the Wald and LM statistics modified with a HAC estimator. In this case, the inferiority of the LM-based tests is especially pronounced. They assessed the relevance of these theoretical results in finite samples via simulations. The results are in contrast to those of Andrews and Ploberger (1994), based on a local asymptotic framework, and revealed its potential weaknesses in the context of structural change problems.

In this paper, we analyze the power of the $q \hat{L} L$ test. We show that for very small breaks, its power is indeed higher than other tests, including the popular sup-Wald test. However, the differences are very minor. Conversely, when the magnitude of change is moderate to large, we show that the power of the test is very low in the context of a regression with lagged dependent variables or when a correction is applied to account for serial correlation in the errors. In many cases, the power goes to zero as the magnitude of change increases. The power of the sup-Wald test does not show this non-monotonicity and its power is far superior to the $q \hat{L} L$ test when the break is not very small. The same can be said of the UDmax test of Bai and Perron (1998). Overall, the results show that the $q \hat{L} L$ test permits very modest gains in power in a static regression with serially uncorrelated errors and small breaks but has serious deficiencies in dynamic models or when a correction for serial correlation is applied. According to the framework EM adopt to define the optimality criterion used, the 
sup-Wald test is not optimal while the $q \hat{L} L$ test is. In this sense, the claims of optimality do not come from the properties of the test statistics but the criterion adopted. Hence, we argue that such a framework to analyze structural change test is inadequate ${ }^{1}$. As in Deng and Perron (2008), we use asymptotic approximations for fixed break sizes to assess the relative efficiency of two tests. We show that the sup-Wald test has higher power than the $q \hat{L} L$ test unless the break size is very small and, in many cases, the latter has zero relative asymptotic efficiency, in the sense that its power goes to zero when the break is large. An earlier version of this paper used the concept of relative Bahadur (1960) efficiency to compare the two tests, as in Kim and Perron (2009). To simplify the exposition we resort to fixedbreak asymptotic approximations. The conclusions are identical since both tests have the same tail behavior and the Bahadur efficiency measure reduces to comparing the limit values (properly scaled) under the alternative hypothesis. We show that the fixed-break asymptotic framework delivers better predictions about the finite sample properties compared to a local asymptotic framework, and that according to this criterion the sup-Wald is more efficient (powerful) in most cases.

It is important to note that we make no claim about any optimality property for the sup-Wald. Andrews and Ploberger (1995) showed that the sup-Wald is optimal (in some sense) against distant alternatives. But this result is of little comfort since they also showed the sup-LM to enjoy the same properties. As shown in Kim and Perron (2009), the power properties of the sup-LM are dramatically inferior to those of the sup-Wald in the context of a regression with lagged dependent variables or when a correction is applied to account for serial correlation in the errors. This discrepancy again occurs because Andrews and Ploberger (1995) also adopt a local asymptotic framework.

The paper is organized as follows. Section 2 presents the model and tests considered. Section 3 motivates the problems to be analyzed with some empirical examples and we show that the $q \hat{L} L$ test fails to reject while the sup-Wald test gives p-values close to zero. Section 4 presents simulation evidence on the finite sample properties of the tests for four types of parameters changes including those used by EM. Section 5 provides an asymptotic comparison of the $q \hat{L} L$ and sup-Wald tests using the fixed-break asymptotic approximations. Section 6 offers brief concluding remarks and an appendix contains technical derivations.

The paper is structured in this manner for the following reasons. First, we wish to convince the readers that the power issues to be tackled are practically relevant. We then

\footnotetext{
${ }^{1}$ For an early example of the potential pitfalls of local asymptotic analyses in the context of tests with non-monotonic power, see Nelson and Savin (1990).
} 
document the main qualitative features via simulations to give an overview of the main issues, so that the readers can get a general overview of the issues, the broad features and their implications. Subsequently, we provide in-depth theoretical explanations of the features documented in the simulations in more general frameworks.

\section{The model and tests considered}

The problem of interest is the following. We have a linear regression model given by:

$$
y_{t}=X_{t}^{\prime} \beta_{t}+e_{t}
$$

for $t=1, \ldots, T$. Under the null hypothesis, the parameter vector $\beta$ of dimension $k$ is constant throughout the sample, i.e., $H_{0}: \beta_{t}=\bar{\beta}$ for all $t$. Under the alternative hypothesis, there is at least one change in $\beta$ occurring within the sample, i.e., $H_{1}: \beta_{t} \neq \bar{\beta}$ for some $t$.

We now describe the test statistics to be analyzed. The $q \hat{L} L$ test statistic of EM is constructed as follows:

Step 1. Compute the OLS residuals $\left\{\hat{e}_{t}\right\}$ from regressing $\left\{y_{t}\right\}$ on $\left\{X_{t}\right\}$.

Step 2. Construct a consistent estimator $\hat{V}_{X}$ of the $k \times k$ long-run covariance matrix of $\left\{X_{t} e_{t}\right\}$. If $e_{t}$ can be assumed serially uncorrelated, a natural choice is a heteroskedasticity robust estimator. For the more general case of possibly autocorrelated errors, many such estimators have been suggested; see, e.g., Newey and West (1987) and Andrews (1991).

Step 3. For each $t=1, \ldots, T$, compute $\left\{\hat{u}_{t}\right\}=\left\{\hat{V}_{X}{ }^{-1 / 2} X_{t} \hat{e}_{t}\right\}$ and denote the $k$ elements of $\left\{\hat{u}_{t}\right\}$ by $\left\{\hat{u}_{t, i}\right\}, i=1, \ldots, k$.

Step 4. For each series $\left\{\hat{u}_{t, i}\right\}$, compute a new series $\left\{\hat{\omega}_{t, i}\right\}$, where $\hat{\omega}_{t, i}=\bar{r} \hat{\omega}_{t-1, i}+\Delta \hat{u}_{t, i}$ and $\hat{\omega}_{1, i}=\hat{u}_{1, i}$, with $\bar{r}=1-c / T$ and $c=10$.

Step 5. Compute the sum of squared residuals from OLS regressions of $\left\{\hat{\omega}_{t, i}\right\}$ on $\left\{\bar{r}^{t}\right\}$ for each $i$ and sum these over $i=1, \ldots, k$.

Step 6. Multiply this sum of sum of squared residuals by $\bar{r}$, and subtract $\sum_{i=1}^{k} \sum_{t=1}^{T} \hat{u}_{t, i}^{2}$ to obtain the test statistic $q \hat{L} L$.

The sup-Wald test statistic is the maximal value of the Wald test statistics over the permissible break dates, i.e., $S W=\sup _{\lambda \in \Lambda_{\epsilon}} W_{T}(\lambda)$, where $\Lambda_{\epsilon}=[\epsilon, 1-\epsilon]$ is the set of the 
possible break fractions. For the linear regression model with i.i.d. errors, the Wald test statistic is given by

$$
W_{T}(\lambda)=T\left(\frac{S S R_{T}^{r}-S S R_{T}(\lambda)}{S S R_{T}(\lambda)}\right)
$$

where $S S R_{T}^{r}$ and $S S R_{T}(\lambda)$ are the restricted and the unrestricted sum of squared residuals, respectively. The latter is obtained from the regression

$$
y_{t}=X_{1}(\lambda)_{t}^{\prime} \beta_{1}+X_{2}(\lambda)_{t}^{\prime} \beta_{2}+e_{t}(\lambda)
$$

where $X_{1}(\lambda)_{t}^{\prime}=X_{t}$ if $t \leq[T \lambda]$ and 0 otherwise, while $X_{2}(\lambda)_{t}^{\prime}=X_{t}$ if $t>[T \lambda]$ and 0 otherwise. When correcting for possible serial correlation in the errors,

$$
W_{T}(\lambda)=\frac{1}{T}\left(\frac{T-2 k}{k}\right) \hat{\beta}^{\prime} H^{\prime}\left(H \hat{V}(\hat{\beta}) H^{\prime}\right)^{-1} H \hat{\beta},
$$

where $\hat{\beta}=\left(\hat{\beta}_{1}, \hat{\beta}_{2}\right)$ is the OLS estimate from $(2), H$ is the conventional matrix such that $(H \hat{\beta})^{\prime}=\left(\hat{\beta}_{1}^{\prime}-\hat{\beta}_{2}^{\prime}\right)$, and $\hat{V}(\hat{\beta})$ is an estimate of the variance covariance matrix of $\hat{\beta}$ that is robust to serial correlation and heteroskedasticity, i.e.,

$$
\hat{V}(\hat{\beta})=T\left(\bar{X}^{\prime} \bar{X}\right)^{-1} \hat{V}_{X}\left(\bar{X}^{\prime} \bar{X}\right)^{-1}
$$

where $\bar{X}=\left[X_{1}(\lambda), X_{2}(\lambda)\right]$. Whenever a correction for serial correlation is needed, we shall use Andrews' (1991) method, so that

$$
\hat{V}_{X}=\hat{\Gamma}_{0}+\sum_{j=1}^{T-1} \kappa(j, m)\left[\hat{\Gamma}_{j}+\hat{\Gamma}_{j}^{\prime}\right]
$$

where $\hat{\Gamma}_{j}=T^{-1} \sum_{t=j}^{T} \hat{v}_{t} \hat{v}_{t-j}^{\prime}$ and $\kappa(j, m)$ is a kernel function with bandwidth $m, \hat{v}_{t}=X_{t} \hat{e}_{t}$ for the $q \hat{L} L$ test and for the sup-Wald test, $\hat{v}_{t}=X_{t} \hat{e}(\lambda)_{t}$, with $\hat{e}(\lambda)_{t}$ the OLS residuals from the regression (2). Below, we use the Bartlett window and choose the bandwidth using the method of Andrews (1991) with an AR(1) approximation. The method implies that $m=1.1447(\alpha T)^{1 / 3}$, where

$$
\alpha=\sum_{i=1}^{k} \frac{4 \hat{\rho}_{i}^{2} \hat{\sigma}_{v i}^{4}}{\left(1-\hat{\rho}_{i}\right)^{6}\left(1+\hat{\rho}_{i}\right)^{2}} / \sum_{i=1}^{k} \frac{\hat{\sigma}_{v i}^{4}}{\left(1-\hat{\rho}_{i}\right)^{4}}
$$

and $\hat{\rho}_{i}$ and $\hat{\sigma}_{v i}^{2}$ are the OLS estimates of the coefficient and error variance from a first-order autoregression applied to $\hat{v}_{i, t}$, i.e. the $i$ th element of $\hat{v}_{t}$ (the results are qualitatively similar using other windows). If only a correction for heteroskedasticity is applied, then $\hat{V}_{X}=\hat{\Gamma}_{0}$. 
Throughout, we use tests with a $5 \%$ nominal size so that with one coefficient allowed to change, the decision rule for the $q \hat{L} L$ is to reject when the statistic is less than 8.36 (see EM) and that for the Wald test, using a trimming $\epsilon=0.15$, is to reject when the statistic is greater than 8.85 (see Andrews, 1993). The choice of the trimming is dictated by the desire to have tests with roughly the same critical values so that the relative power properties can be properly evaluated in the theoretical analysis given that we compare the power of the tests with respect to the limit of the statistics for fixed values of the parameters under the alternative. This is made for convenience in order to allow an easier evaluation of the theoretical predictions about the relative power functions.

\section{Motivation}

It is useful to start with empirical examples to illustrate the main issues involved. We first consider the US (ex-post) real interest rate series over the period 1961:1-1986:3 (the threemonth treasury bill rate, deflated by the CPI inflation rate taken from the Citibase data bank). It is the same series analyzed by Garcia and Perron (1996) and Bai and Perron (2003). We reproduce the series in Figure 1. Garcia and Perron (1996) argued that the series was basically a white noise process around a mean that exhibited two changes, one in 1972:3 and one in 1980:3. Using more sophisticated procedures, Bai and Perron (2003) argued for the presence of a third small break in 1966:4. As can be seen from the figure, the change in 1980:3 is very large (7.44\%), the change in 1972:3 is moderate $(2.67 \%)$ and the change in 1966:4 is small (0.95\%). This series therefore fits the framework of EM quite well, in the sense that one can view the changes in mean occurring with some small probability and random magnitude.

This is a simple problem of changes in mean so that the relevant regressor is a constant. It is important first to check if the residuals are correlated under the null hypothesis since this dictates the appropriate versions of the tests to use. To do so here, one must simply assess whether the demeaned series shows evidence of autocorrelation. The Lagrange Multiplier test statistic for serial correlation up to order four is highly significant with a value of 54.42 (the first-order serial correlation of the estimated residuals is 0.628). We therefore need to correct any statistic used for the presence of serial correlation in the errors. We apply nonparametric corrections, the so-called robust versions of the tests as described in the previous section. The value of the $q \hat{L} L$ test is -6.51 , which is insignificant, even at the $10 \%$ level. On the other hand, the value of the sup-Wald test is 74.50 , which is highly significant with a 
p-value ${ }^{2}$ less than $0.001^{3}$.

Another example of similar results can be found in Giacomini and Rossi (2006). They considered testing for structural change in a regression of the growth rate of industrial production on the difference between long-term and short-term interest rates. Their model implies serially correlated errors so that a correction is needed. Even though they fix the bandwidth a priori, the results show overwhelming rejections with Wald-type tests and nonrejections with the $q \hat{L} L$ and LM-type tests.

Consider now an example involving a regression with a lagged dependent variable so that if the model is well specified, the errors are uncorrelated and there is no need for a correction. Musso, Stracca and van Dick (2009) study instability and nonlinearity in the Euro Area Phillips curve. A specification they consider is the following simple model:

$$
\pi_{t}=\mu+\alpha \pi_{t-1}+\beta x_{t}+e_{t}
$$

where $\pi_{t}$ is the inflation rate (here the GDP Deflator) and $x_{t}$ is a measure of the output gap 4 (the data are quarterly for the period 1970:1 and 2005:4, see Musso et al., 2009, for more details $^{5}$ ). Lags of $\Delta \pi_{t}$ and $\Delta x_{t}$ can be added but the results reported below are qualitatively similar. Using a time varying parameter model, Musso et al. (2009) argue that a large change in the mean of inflation $\mu$ and a small change in the slope parameter $\beta$ have occurred, and that once these changes are accounted for, there is no evidence of changes in the persistence parameter $\alpha$. We applied the $q \hat{L} L$ and sup-Wald tests to this regression to test for a change in $\mu$. The $q \hat{L} L$ test is unable to reject the null hypothesis of no change (the value is -5.255 , so that the p-value is well above $10 \%$ ). On the contrary, and in accordance with the results of Musso et al. (2009), the sup-Wald test overwhelmingly rejects the null hypothesis (the value is 129.31, so that the p-value is well below 0.001). With the specification (4), neither of the tests are able to reject the null hypothesis of no change in $\beta$. However, if three lags of $\Delta \pi_{t}$ are added to the regression, both reject at the $5 \%$ level despite the fact that the change is modest (the values are -14.192 for the $q \hat{L} L$ and 10.57 for sup-Wald).

\footnotetext{
${ }^{2}$ Throughout, p-value refers to "asymptotic p-value".

${ }^{3}$ The value of the sup-Wald test for two changes considered in Garcia and Perron (1996) and Bai and Perron (1998) is 48.26, also highly significant. The UDmax test of Bai and Perron (1998) is also highly significant with a value of 56.53 .

${ }^{4}$ We used the first principle component of the following six indicators: the three versions of the output gap estimates based on the production function approach using three varieties of unobserved components models and the three types of filtered real GDP estimates using three different filters. See Musso et al. (2007) for more details, who also use other measures. We also tried, as they suggest, a simple average of these six indicators with no change in qualitative results.

${ }^{5}$ We are grateful to Dick van Dijk for supplying us with the data.
} 
The goal of this paper is to explain why in all three examples, the $q \hat{L} L$ test has no power against large parameter changes while the sup-Wald has high power and why both have similar power against small changes.

\section{Some simulation results}

We start with simulation experiments to illustrate the problems to be addressed. The simulations are based on a simple regression with one regressor $(k=1)$. The data generating process is

$$
y_{t}=\mu+x_{t} \beta_{t}+e_{t}
$$

with $e_{t} \sim N\left(0, \sigma_{e}\right)$, and where the regressor $x_{t}$ is a simple $\operatorname{AR}(1)$ process of the form

$$
\begin{aligned}
& x_{t}=z_{t}+\kappa, \\
& z_{t}=\rho z_{t-1}+v_{t},
\end{aligned}
$$

with $v_{t} \sim$ i.i.d. $N\left(0, \sigma_{v}\right)$. We set $\kappa=5, \mu=0, \rho=0.5$ and $\sigma_{e}=\sigma_{v}=1$ in all cases. The results are robust to variations in the DGP but it is important that the mean of the regressor $\kappa$ be non-zero. We consider the power of the $q \hat{L} L$ test, the sup-Wald test, the UDmax test of Bai and Perron (1998), as well as Nyblom's (1989) test $N y$ defined by

$$
N y=\frac{1}{T^{2} \hat{\sigma}_{e}^{2}} \operatorname{tr}\left[Q_{T}^{-1} \sum_{t=1}^{T} S_{t} S_{t}^{\prime}\right]
$$

where $S_{t}=\sum_{j=t}^{T} X_{j} \hat{e}_{j}, Q_{T}=T^{-1} \sum_{t=1}^{T} X_{t} X_{t}^{\prime}$, and $\hat{\sigma}_{e}^{2}=T^{-1} \sum_{t=1}^{T} \hat{e}_{t}^{2}$. We consider four types of parameter variations for the coefficient $\beta_{t}$, given by the following models:

$$
\begin{array}{ll}
\text { Model A }: & \beta_{t}=\beta_{t-1}+\varepsilon_{t}, \quad \varepsilon_{t} \sim \text { i.i.d.N }(0, \sigma), \beta_{0}=0, \\
\text { Model B : } & \beta_{t}=\sum_{j=2}^{[T / 20]} \eta_{j} I_{[20(j-1)<t \leq 20 j]}, \quad \eta_{j} \sim \text { i.i.d. } N(0, \sigma), \\
\text { Model C : } & \beta_{t}=\eta I_{t>\left[T \lambda_{c}^{r}\right]}, \quad \eta \sim i . i . d . \quad N(0, \sigma), \\
\text { Model D : } & \beta_{t}=\theta I_{t>\left[T \lambda_{c}\right]},
\end{array}
$$

where $j=1, \ldots,[T / 20], \lambda_{c}^{r}$ is uniformly distributed on the unit interval, and $\lambda_{c}=0.5$. Model A specifies a random walk behavior for the coefficient; Model B, a break of random magnitude every 20 observations; Model C, a one time break of some random magnitude at some random date; and Model $\mathrm{D}$, a one time break of fixed magnitude $\theta$ at a fixed date 
$\left[T \lambda_{c}\right]$. Note that Models A, B, and $\mathrm{C}$ can be viewed as satisfying the conditions stated in EM for the $q \hat{L} L$ test to be viewed as optimal. Here, the parameter that indexes the magnitude of the break is $\sigma^{2}$, the variance of the shocks affecting $\beta_{t}$. Models $\mathrm{B}$ and $\mathrm{C}$ are those used by EM to document the finite sample performance of their test. Model $\mathrm{D}$ does not satisfy the conditions for the $q \hat{L} L$ to be optimal given that the break is non-random, but we include it since the sup-Wald test is especially designed for this case. Unless otherwise indicated, $T=100$ and throughout, the results are obtained from 3,000 replications. We report size-adjusted power. The exact size of the $q \hat{L} L$ is, in general, close to the nominal size. On the other hand, the sup-Wald sometimes exhibit liberal size distortion. This feature is similar to that documented by Vogelsang (1999) who compared the properties of the supLM and sup-Wald tests when testing for a change in mean. Given the power results to be reported, this does not imply a meaningful size-power trade-off between the two tests given the non-monotonic power of the $q \hat{L} L$.

We compute the power function of four tests: the $q \hat{L} L$, the sup-Wald, Nyblom's test (5\% critical value 0.461), and the UDmax test of Bai and Perron (1998) with the maximum number of breaks set to 5 (with a $15 \%$ trimming so that the $5 \%$ critical value is 8.88 ). We consider three versions for each test: a) the "static case" for which the investigator assumes a priori that the errors are serially uncorrelated and homoskedastic and hence, the regression is (5) and the non-robust versions of the tests are used ${ }^{6}$; b) the "robust case" for which no such assumption about the nature of serial correlation in the errors is made and, hence, the robust versions of the tests are used, as described in Section 2; and c) the "dynamic case" where a lagged dependent variable is included, either as part of the model or to account for serial correlation in a more parametric fashion and the non-robust versions of the tests are applied to the regression

$$
y_{t}=\alpha y_{t-1}+x_{t} \beta_{t}+e_{t} .
$$

The fact that we set $\alpha=0$ and, hence, that the lagged dependent variable is an irrelevant regressor is completely inconsequential. With a non-zero value, the power loss of the $q \hat{L} L$ reported below would simply occur earlier and be exacerbated.

The results for Models A and B are presented in Figure 2, while those for Models C and $\mathrm{D}$ are presented in Figure 3. We plot the power of the tests as a function of $\sigma$ for Models $\mathrm{A}, \mathrm{B}$, and $\mathrm{C}$, and as a function of $\theta$ for Model D. Consider first the static case presented in the top panels. For Models A, B, and C with very small breaks, the test $q \hat{L} L$ indeed has

\footnotetext{
${ }^{6}$ We also considered assuming a priori no serial correlation but applying a correction for heteroskedasticity. The results are similar to those of the "static case" and, hence, are not reported.
} 
the highest power. For larger breaks, the power of all tests is basically the same. For Model $\mathrm{D}$, the sup-Wald is slightly more powerful, as expected. But again the differences are minor and vanish as the magnitude of the break increases. The results for Models $\mathrm{B}$ and $\mathrm{C}$ are consistent with those reported in EM. It should be noted that the differences between the $q \hat{L} L$ and the sup-Wald tests are very minor so one could draw the conclusion that, though not a member of the family of optimal tests as considered by EM, the sup-Wald test is nevertheless nearly as efficient.

Things are very different when considering the robust versions of the tests, which correct for potential serial correlation. These results are presented in the second panels. In all cases, the power of the $q \hat{L} L$ test initially increases with the magnitude of the breaks but quickly decreases (sometimes to zero) subsequently. This is also the case for Nyblom's test. But the power of the sup-Wald test does not show this non-monotonicity and its power is far superior to the $q \hat{L} L$ test when the break is not very small. The same can be said of the UDmax test, which has the best overall performance. Similar results are obtained when considering the "dynamic case" in which the regression has a lagged dependent variable, given in the third panel.

Overall, the results show that the $q \hat{L} L$ test permits modest gains in power in the "static case" but has serious deficiencies in dynamic models or when correcting for serial correlation. Some may argue that the relevant case is that of the "static regression". But we argue this is not so. Indeed, a careful investigator would rarely use a static regression with no correction for serial correlation because if breaks are present, the estimated residuals will exhibit serial correlation. Take the simple case of a single change, random or fixed. Then the residuals will exhibit a change in mean. As shown in Perron (1990), this change will cause an increase in the sum of the autoregressive coefficients in an autoregression fitted to the series (here the fitted residuals). Hence, a test for serial correlation applied to the estimated residuals will be significant unless the break is very small. A similar logic applies to multiple breaks, including a random walk process. To document this, Figures 4 and 5 present the power of the LM test for serial correlation up to order 4 in the residuals for each of the four models when estimating the static regression. In all cases, the power increases as the magnitude of the breaks increases. We also present the mean of the estimates of the autoregressive coefficient in an AR(1) model applied to the fitted residuals. The results show that it increases rapidly and flattens out at a level below one (see Perron, 1990, for an explanation in the single break case). Thus, an investigator who has no prior knowledge about the presence or absence of serial correlation in the residuals would, upon performing a test for serial correlation, 
conclude that the robust version is needed when the breaks are not very small. The top panels of Figures 4 and 5 present the power of the hybrid version of the $q \hat{L} L$ test. By "hybrid", we mean that if a test for serial correlation in the residuals does not reject, the non-robust version is used, otherwise the robust version is used. As expected, the power initially increases but quickly drops as the magnitude of change increases. So the good finite sample performance of the $q \hat{L} L$ test as reported in EM crucially depends on prior knowledge that the errors are not serially correlated, knowledge that is unavailable in practice.

These simulation results go a long way in explaining the empirical findings reported in the previous section. For the real interest rate series, there is at least one very large change in mean. This break contaminates the estimated residuals when fitting a restricted model that allows only for an intercept, as required to construct the $q \hat{L} L$ test. Accordingly, it biases the estimate of the long-run variance to correct for serial correlation in a way that reduces power to a very low level. The simulation results also explain the lack of power when the regressors include a lagged dependent variable and the break is large, as in the example discussed in Section 3. The loss of power in the case of a model with a lagged dependent variable is especially interesting. It shows that the problem of the $q \hat{L} L$ does not occur solely because it does not get the right scaling pertaining to get the correction for serial correlation as in the robust case. The sup-Wald does not get it right either (except for a one time break) and yet does not suffer from such problems in both the robust and dynamic cases.

As shown by the simulations, the break need not be "very large" for the decline in the power of the $q \hat{L} L$ to occur. In general, the reversal occurs before the power obtained assuming serially uncorrelated errors and no lagged dependent variable reaches one. But as will be shown in the theoretical section, when applying the test for changes in the intercept (as when testing for a change in mean), the power reversal occurs earlier and is even more pronounced. Hence, the problem applies to an empirically relevant part of the parameter space, as documented in the various examples discussed in Section 3.

In the next section, we provide a theoretical analysis of the properties of the $q \hat{L} L$ and supWald tests based on fixed-break asymptotic approximations. These will show that indeed, in all cases, the $q \hat{L} L$ test has zero asymptotic efficiency compared to the sup-Wald test when a correction for serial correlation in the errors is applied or when a dynamic regression is involved. With a static regression and the prior knowledge of no serial correlation, the two tests have similar properties and in some cases, the sup-Wald actually dominates. 


\section{$5 \quad$ Asymptotic comparisons using fixed-break asymptotic approximations}

We carry the theoretical analysis for the random walk Model A, for random level shift models of the parameters (Models B and C), and for parameters with a one-time change of nonrandom magnitude at some fixed date (Model D). Throughout, we shall maintain (1) as the data-generating process and impose the following high-level assumptions on the regressors and errors as in Kim and Perron (2009), where as a matter of notation, $\|X\|$ denotes the $L_{2}$-norm of a random matrix $X$, i.e., $\|X\|=\left(\sum_{i} \sum_{j} E\left|X_{i j}\right|^{2}\right)^{1 / 2}$.

Assumption 1 Let $v_{t}=X_{t} e_{t}, p \lim _{T \rightarrow \infty} T^{-1} \sum_{t=j+1}^{T} E\left(v_{t} v_{t-j}^{\prime}\right)=\Gamma_{j}$, and $\Sigma=\Gamma_{0}+\sum_{j=1}^{\infty}\left(\Gamma_{j}+\right.$ $\left.\Gamma_{j}^{\prime}\right)$. Then $E\left(v_{t}\right)=0, \sup _{j \in\left\{0, \ldots, L_{T}\right\}} \sup _{r \in[0,1]}\left\|T^{-1} \sum_{t=1}^{[T r]} v_{t} v_{t-j}^{\prime}-r \Gamma_{j}\right\|=o_{p}(1)$ for $L_{T}=$

$o(T)\left(\Gamma_{-j}=\Gamma_{j}^{\prime}\right)$, and the partial sums of $v_{t}$ satisfy the functional central limit theorem $T^{-1 / 2} \sum_{t=1}^{[T r]} v_{t} \Rightarrow \Sigma^{1 / 2} W_{k}(r)$, where $W_{k}(r)$ is a k-dimensional Wiener process defined on $[0,1]$. Note that if $v_{t}$ is uncorrelated, then $\Sigma=\Gamma_{0}$.

Assumption 2 The regressors are such that

$$
\sup _{j \in\left\{0, \ldots, L_{T}\right\}} \sup _{r \in[0,1]}\left\|T^{-1} \sum_{t=j+1}^{[T r]} X_{t} X_{t-j}^{\prime}-r Q_{j}\right\|=o_{p}(1),
$$

where $L_{T}=o(T), Q_{-j}=Q_{j}^{\prime}$, and $Q_{j}$ is some non-singular fixed matrix bounded uniformly in j, i.e., $\sup _{|j| \leq L_{T}}\left\|Q_{j}\right\| \leq c<\infty$. We denote $Q_{0}$ by $Q$ for simplicity.

Assumption $3 T^{-1} \sum_{t=1}^{T}\left\|X_{t} X_{t}^{\prime}\right\|^{2}=O_{p}(1)$, and with $L_{T}=o(T)$,

$$
\begin{gathered}
\sup _{r \in[0,1]}\left\|T^{-1} \sum_{t=j+1}^{[T r]} X_{t} X_{t}^{\prime} X_{t} X_{t}^{\prime}-r M M^{\prime}\right\|=o_{p}(1), \\
\sup _{j \in\left\{0, \ldots, L_{T}\right\}} \sup _{r \in[0,1]}\left\|T^{-1} \sum_{t=j+1}^{[T r]} X_{t} X_{t}^{\prime} X_{t} X_{t-j}^{\prime}-r M_{j} M_{j}^{\prime}\right\|=o_{p}(1), \\
\sup _{j \in\left\{0, \ldots, L_{T}\right\}} \sup _{r \in[0,1]}\left\|T^{-1} \sum_{t=j+1}^{[T r]} X_{t} X_{t}^{\prime} X_{t-j} X_{t-j}^{\prime}-r M_{j j} M_{j j}^{\prime}\right\|=o_{p}(1),
\end{gathered}
$$

where $M, M_{j}$, and $M_{j j}$ are some fixed matrices bounded uniformly in $j$.

\subsection{The random walk model}

In this first case, we suppose that $\beta_{t}$ is generated by a random walk process, i.e.,

$$
\beta_{t}=\beta_{t-1}+\varepsilon_{t},
$$


with $\varepsilon_{t}$ is a $k$-dimensional i.i.d. vector process with $E\left(\varepsilon_{t}\right)=0, E\left(\varepsilon_{t} \varepsilon_{t}^{\prime}\right)=\Omega$ and $E\left(\varepsilon_{t} e_{t-j}\right)=0$ for all $j$. Also, $\varepsilon_{t}$ is uniform mixing with mixing coefficient $\phi$ of size $-r /(2 r-2)$ or strong mixing with mixing coefficient $\alpha$ of size $-r /(r-2), r>2$. We also assume that $\varepsilon_{t}$ and $X_{t}$ are uncorrelated so that the following functional central limit theorem holds:

$$
T^{-3 / 2} \sum_{t=1}^{[T r]} X_{t} X_{t}^{\prime} \beta_{t} \Rightarrow Q \Omega \int_{0}^{r} W(s) d s,
$$

where $W(s)$ is the $k$-dimensional standard Wiener process and " $\Rightarrow$ " denotes weak convergence under the Skorohod topology. We also define the Ornstein-Uhlenbeck process $W_{c}(s) \equiv W(s)-c \int_{0}^{s} e^{-c(s-\lambda)} W(\lambda) d \lambda$. We start with the case of the static regression for which the investigator has prior knowledge that the errors are serially uncorrelated. Note that here and throughout the paper, the symbol $O_{p}(1)$ stands for a variable that is stochastically bounded but is not $o_{p}(1)$.

Theorem 1 Assume that the $q \hat{L} L$ and sup-Wald $(S W)$ tests are constructed from the static regression (5) with no correction for serial correlation and that the data are generated by (1) with $\beta_{t}$ specified by the random walk (8), then:

$$
T^{-1} q \hat{L} L \Rightarrow \operatorname{tr}\left[\bar{V}_{X}^{-1 / 2} Q \Omega \Xi \Omega^{\prime} Q^{\prime} \bar{V}_{X}^{\prime-1 / 2}\right]=O_{p}(1)
$$

uniformly in $\|\Omega\|$, where

$$
\bar{V}_{X} \equiv M \Omega\left[\int_{0}^{1} W W^{\prime}-\int_{0}^{1} W \int_{0}^{1} W^{\prime}\right] \Omega^{\prime} M^{\prime}
$$

and

$$
\Xi \equiv \int_{0}^{1} W_{c} W_{c}^{\prime}-\int_{0}^{1} W W^{\prime}+\int_{0}^{1} W \int_{0}^{1} W^{\prime}-\frac{2 c}{1-e^{-2 c}} \int_{0}^{1} e^{-c r} W \int_{0}^{1} e^{-c r} W^{\prime}
$$

Also,

$$
T^{-1} S W \Rightarrow \frac{S S R^{r *}-S S R^{*}(\bar{\lambda})}{S S R^{*}(\bar{\lambda})}=O_{p}(1)
$$

uniformly in $\|\Omega\|$, where

$$
\begin{aligned}
S S R^{r *} & \equiv \int_{0}^{1} W^{\prime} \Omega^{\prime} Q \Omega W-\int_{0}^{1} W^{\prime} \Omega^{\prime} Q \Omega \int_{0}^{1} W \\
S S R^{*}(\lambda) & \equiv \int_{0}^{1} W^{\prime} \Omega^{\prime} Q \Omega W-\frac{1}{\lambda} \int_{0}^{\lambda} W^{\prime} \Omega^{\prime} Q \Omega \int_{0}^{\lambda} W-\frac{1}{1-\lambda} \int_{\lambda}^{1} W^{\prime} \Omega^{\prime} Q \Omega \int_{\lambda}^{1} W
\end{aligned}
$$

and $\bar{\lambda}$ is the value of $\lambda$ that minimizes $\operatorname{SSR}^{*}(\lambda)$ over the set $[\epsilon, 1-\epsilon]$. 
Consider the case with a single regressor that is a constant. The tests then amount to distinguishing between an i.i.d. process and a $I M A(1,1)$ process. The expressions in Theorem 1 reduce to

$$
T^{-1} q \hat{L} L \Rightarrow\left[\int_{0}^{1} W^{2} d r-\left(\int_{0}^{1} W d r\right)^{2}\right]^{-1} \Xi
$$

and

$$
T^{-1} S W \Rightarrow \frac{S S R^{r *}-S S R^{*}(\bar{\lambda})}{S S R^{*}(\bar{\lambda})}
$$

where

$$
\begin{aligned}
S S R^{*}(\lambda) & =\int_{0}^{1} W^{2} d r-\frac{1}{\lambda}\left(\int_{0}^{1} W d r\right)^{2}+\frac{2}{\lambda} \int_{0}^{1} W d r \int_{\lambda}^{1} W d r-\frac{1}{\lambda(1-\lambda)}\left(\int_{\lambda}^{1} W d r\right)^{2} \\
S S R^{r *} & =\int_{0}^{1} W^{2} d r-\left(\int_{0}^{1} W d r\right)^{2}
\end{aligned}
$$

and again $\bar{\lambda}$ is the value of $\lambda$ that minimizes $S S R^{*}(\lambda)$ over the set $[\epsilon, 1-\epsilon]$.

Note that, appropriately scaled, the limits of the $q \hat{L} L$ and sup-Wald tests are random in this case. Also, in the case of a single regressor, they do not depend on any nuisance parameters. This is because in the limit, the random walk component dominates. Since the power depends on the relative variances of the shocks $e_{t}$ and those affecting $\beta_{t}$, we should not expect a good finite sample approximation for small breaks. Nevertheless, it is instructive to compare the mean of the limit random variables in both cases. To that effect, we simulated the random variables in (9) and (10) using 1,000 steps to approximate the Wiener process and 1,000 replications. The negative of the mean of the random variable in (9) is 1.13 . The mean of the random variable in (10) depends on the trimming used to search for the break dates that minimizes the sum of squared residuals. For $\epsilon=0.15$, the value is 2.01. We performed a similar analysis of the DGP used in the simulations of Section 4. In this case, the mean of the limit distribution of the sup-Wald test is essentially the same, while the mean of the limit distribution of the $q \hat{L} L$ test is somewhat smaller at 0.76 . These values are large enough to imply that both tests reject when $T=100$ at a size of $5 \%$. The cdf of the limit distribution of the sup-Wald test almost uniformly dominates that of the $q \hat{L} L$ test even when $\Omega=1$, a small value (the limiting cdf of (minus) the $q \hat{L} L$ test is to the right only for the extreme left tail). But the numerical results predict that both tests will have good (and similar) finite sample power as documented in the simulations.

We now turn to the case where one accounts for possible serial correlation in the errors using a non-parametric correction. In the case of the $q \hat{L} L$ test, this amounts to replacing the variance estimate $\hat{V}_{X}$ by $\hat{h(0)}$, i.e., an estimate of the spectral density function at frequency 
zero. Typically, it is obtained using a weighted sum of the autocovariances of $\left\{\hat{v}_{t}=X_{t} \hat{e}_{t}\right\}$ so that

$$
\hat{h(0)}=\hat{\Gamma}_{0}+\sum_{j=1}^{T-1} \kappa(j, m)\left[\hat{\Gamma}_{j}+\hat{\Gamma}_{j}^{\prime}\right]
$$

where $\hat{\Gamma}_{j}=T^{-1} \sum_{t=j}^{T} \hat{v}_{t} \hat{v}_{t-j}^{\prime}$ and $\kappa(\cdot)$ is a kernel function with bandwidth $m$. We use the Bartlett kernel for $\kappa(\cdot)$ and Andrews' (1991) data dependent method for selecting the bandwidth based on an $\mathrm{AR}(1)$ approximation. Then, the following results hold.

Theorem 2 Assume that the $q \hat{L} L$ and sup-Wald $(S W)$ tests are constructed from the static regression (5) and that the process is generated by (1) with $\beta_{t}$ specified by the random walk (8). Suppose serial correlation in the errors is accounted for using an estimate of the form (11) with the Bartlett kernel and the bandwidth chosen by Andrew's (1991) data dependent method based on an $A R(1)$ approximation. a) If $\left\{M-M_{11}\right\}_{i i}=0$ for some $i$, where $\{Z\}_{i i}$ is the ith diagonal element of the matrix $Z$, then

$$
q \hat{L} L \Rightarrow \operatorname{tr}\left[\bar{h}(0)^{-1 / 2} Q \Omega \Xi \Omega^{\prime} Q^{\prime} \bar{h}(0)^{\prime-1 / 2}\right]=O_{p}(1)
$$

uniformly in $\|\Omega\|$, where $\Xi$ is as defined in Theorem 1,

$$
\bar{h}(0) \equiv \bar{\Gamma}_{0}+\lim _{T \rightarrow \infty} T^{-1} \sum_{j=1}^{T} \kappa(j, m)\left[\bar{\Gamma}_{j}+\bar{\Gamma}_{j}^{\prime}\right]
$$

with $\bar{\Gamma}_{j} \equiv M_{j j} \Omega\left[\int_{0}^{1} W W^{\prime}-\int_{0}^{1} W \int_{0}^{1} W^{\prime}\right] \Omega^{\prime} M_{j j}^{\prime}, m \equiv 1.1447(\bar{\alpha} T)^{1 / 3}$, and $\bar{\alpha} \equiv \sum_{i=1}^{k} \phi_{i}^{-2}$ with

$$
\phi_{i} \equiv \frac{-W_{i}(1)^{2}-\left(\int_{0}^{1} W_{i}\right)^{2}+W_{i}(1) \int_{0}^{1} W_{i}}{\int_{0}^{1} W_{i}^{2}-\left(\int_{0}^{1} W_{i}\right)^{2}},
$$

for $i$ such that $\left\{M-M_{11}\right\}_{i i}=0$, and 0 otherwise, where $W_{i}(i=1, \ldots, k)$ is the ith element of $W$. Also,

$$
\begin{aligned}
S W \Rightarrow & \left(\frac{1}{\lambda^{*}} \Omega \int_{0}^{\lambda^{*}} W-\frac{1}{1-\lambda^{*}} \Omega \int_{\lambda^{*}}^{1} W\right)^{\prime} Q^{\prime}\left[\bar{h}_{1}(0)+\bar{h}_{2}(0)\right]^{-1} Q \\
& \times\left(\frac{1}{\lambda^{*}} \Omega \int_{0}^{\lambda^{*}} W-\frac{1}{1-\lambda^{*}} \Omega \int_{\lambda *}^{1} W\right)=O_{p}(1)
\end{aligned}
$$

uniformly in $\|\Omega\|$, where $\lambda^{*}$ is the value of $\lambda$ that maximizes the limit of the Wald test over the set $[\epsilon, 1-\epsilon], \bar{h}_{l}(0) \equiv \bar{\Gamma}_{l, 0}+\lim _{T \rightarrow \infty} T^{-1} \sum_{j=1}^{T} \kappa\left(j, m_{l}\right)\left[\bar{\Gamma}_{l, j}+\bar{\Gamma}_{l, j}^{\prime}\right] \quad(l=1,2)$, with

$$
\begin{aligned}
\bar{\Gamma}_{1, j} & \equiv M_{j j} \Omega\left[\int_{0}^{\lambda^{*}} W W^{\prime}-\int_{0}^{\lambda^{*}} W \int_{0}^{\lambda^{*}} W^{\prime}\right] \Omega^{\prime} M_{j j}^{\prime}, \\
\bar{\Gamma}_{2, j} & \equiv M_{j j} \Omega\left[\int_{\lambda^{*}}^{1} W W^{\prime}-\int_{\lambda^{*}}^{1} W \int_{\lambda^{*}}^{1} W^{\prime}\right] \Omega^{\prime} M_{j j}^{\prime},
\end{aligned}
$$




$$
\begin{aligned}
m_{l} \equiv 1.1447\left(\bar{\alpha}_{l} T\right)^{1 / 3}, \bar{\alpha}_{l} \equiv \sum_{i=1}^{k} \phi_{l, i}^{-2}, \text { and } \\
\phi_{1, i} \equiv \frac{-W_{i}\left(\lambda^{*}\right)^{2}-\left(\int_{0}^{\lambda^{*}} W_{i}\right)^{2}+W_{i}\left(\lambda^{*}\right) \int_{0}^{\lambda^{*}} W_{i}}{\left(\int_{0}^{\lambda *} W_{i}^{2}\right)-\left(\int_{0}^{\lambda^{*}} W_{i}\right)^{2}}, \\
\phi_{2, i} \equiv \frac{-\left(W_{i}(1)-W_{i}\left(\lambda^{*}\right)\right)^{2}-\left(\int_{\lambda^{*}}^{1} W_{i}\right)^{2}+\left(W_{i}(1)-W_{i}\left(\lambda^{*}\right)\right) \int_{\lambda^{*}}^{1} W_{i}}{\int_{\lambda^{*}}^{1} W_{i}^{2}-\left(\int_{\lambda^{*}}^{1} W_{i}\right)^{2}},
\end{aligned}
$$

for $i$ such that $\left\{M-M_{11}\right\}_{i i}=0$, and 0 otherwise. b) If $\left\{M-M_{11}\right\}_{i i} \neq 0$ for all $i$, then

$$
T^{-2 / 3} q \hat{L} L \Rightarrow \operatorname{tr}\left[\bar{h}(0)^{-1 / 2} Q \Omega \Xi \Omega^{\prime} Q^{\prime} \bar{h}(0)^{\prime-1 / 2}\right]=O_{p}(1)
$$

uniformly in $\|\Omega\|$, where $\bar{h}(0) \equiv \bar{\Gamma}_{0}+\lim _{T \rightarrow \infty} T^{-1 / 3} \sum_{j=1}^{T} \kappa\left(j, m_{T}\right)\left[\bar{\Gamma}_{j}+\bar{\Gamma}_{j}^{\prime}\right]$, with $\bar{\Gamma}_{j}$, $\Xi$ and $m_{T}$ as defined in part (a), and

$$
\bar{\alpha} \equiv \sum_{i=1}^{k} \frac{4 \bar{\rho}_{i}^{2} \bar{\sigma}_{v i}^{4}}{\left(1-\bar{\rho}_{i}\right)^{6}\left(1+\bar{\rho}_{i}\right)^{2}} / \sum_{i=1}^{k} \frac{\bar{\sigma}_{v i}^{4}}{\left(1-\bar{\rho}_{i}\right)^{4}}
$$

with $\bar{\rho}_{i} \equiv\left\{M_{11}\right\}_{i i}^{2} /\{M\}^{2}$ and $\bar{\sigma}_{v i}^{2}=p \lim T^{-1} \sum_{t=2}^{T}\left(\hat{v}_{i t}-\hat{\rho}_{i} \hat{v}_{i t-1}\right)^{2}$. Also,

$$
\begin{aligned}
T^{-2 / 3} S W \Rightarrow & \left(\frac{1}{\lambda^{*}} \Omega \int_{0}^{\lambda^{*}} W-\frac{1}{1-\lambda^{*}} \Omega \int_{\lambda^{*}}^{1} W\right)^{\prime} Q^{\prime}\left[\bar{h}_{1}(0)+\bar{h}_{2}(0)\right]^{-1} Q \\
& \times\left(\frac{1}{\lambda^{*}} \Omega \int_{0}^{\lambda^{*}} W-\frac{1}{1-\lambda^{*}} \Omega \int_{\lambda *}^{1} W\right)=O_{p}(1)
\end{aligned}
$$

uniformly in $\|\Omega\|$, where $\lambda^{*}$ is the value of $\lambda$ that maximizes the limit of the Wald test over the set $[\epsilon, 1-\epsilon], \bar{h}_{l}(0) \equiv \bar{\Gamma}_{l, 0}+\lim _{T \rightarrow \infty} T^{-1 / 3} \sum_{j=1}^{T} \kappa\left(j, m_{l}\right)\left[\bar{\Gamma}_{l, j}+\bar{\Gamma}_{l, j}^{\prime}\right](l=1,2)$, with $\bar{\Gamma}_{l, j}$, $m_{l}$ as defined in part (a), and

$$
\bar{\alpha}_{l} \equiv \sum_{i=1}^{k} \frac{4 \bar{\rho}_{l, i}^{2} \bar{\sigma}_{v i, l}^{4}}{\left(1-\bar{\rho}_{l, i}\right)^{6}\left(1+\bar{\rho}_{l, i}\right)^{2}} / \sum_{i=1}^{k} \frac{\bar{\sigma}_{v i, l}^{4}}{\left(1-\bar{\rho}_{l, i}\right)^{4}},
$$

where $\bar{\rho}_{l, i}$, and $\bar{\sigma}_{v i, l}^{2}$ are as defined for the limit of the $q \hat{L} L$ test.

Though the results are quite complex, we can simulate these limit distributions. Note that we approximate $\bar{h}(0)$ with a scaled average where $T=100$. Similarly $m$ is evaluated at $T=100$. We ran simulations for the DGP considered in the simulations of Section 4, as specified by (5). This is a case where part (b) of Theorem 2 applies since it involves a single regressor and $M \neq M_{11}$. The means, as a function of $\Omega$, are presented in Figure 6 . The figure shows that the limit is invariant to $\Omega$, as in the static case with i.i.d. errors. But, we can use these limit results to see what the theory predicts about the likelihood of a rejection. For a 
given sample size $T$, a rejection will occur if the realization of the limit random variable is above the critical values divided by $T^{2 / 3}$ (using $-q \hat{L} L$ ). As in the simulations, we considered $5 \%$ tests. The relevant critical values are plotted as the dotted line in each panel. The results predict that on average the $q \hat{L} L$ test will not reject, while the sup-Wald will. To get a better description in terms of the probability of rejecting, the lower panels in Figure 6 present the cdfs of the limit random variables. The results clearly show that the $q \hat{L} L$ test has a very small probability of rejecting when $\Omega=1$ and nearly none if $\Omega=15$, while the sup-Wald test has large rejection probabilities in both cases. These theoretical predictions are in accordance with the simulations. Though, these results are DGP specific, we found that the same features hold for other specifications.

Suppose now that we use a dynamic regression of the form (6) with a lagged dependent variable as a regressor. The results are stated in the next theorem.

Theorem 3 Assume that the $q \hat{L} L$ and sup-Wald $(S W)$ tests are constructed from a dynamic regression of the form (6), with a lagged dependent variable as a regressor, and the process is generated by (1), with $\beta_{t}$ specified by the random walk (8). Then,

$$
T^{-1} q \hat{L} L \Rightarrow \operatorname{tr}\left[\bar{V}_{X}^{-1 / 2}\left[Q-\bar{\alpha} Q_{1}\right] \Omega \Xi \Omega^{\prime}\left[Q-\bar{\alpha} Q_{1}\right]^{\prime} \bar{V}_{X}^{\prime-1 / 2}\right]=O_{p}(1)
$$

uniformly in $\|\Omega\|$, where $\Xi$ is as defined in Theorem 1,

$$
\bar{\alpha} \equiv\left\{\int_{0}^{1} W^{\prime} \Omega^{\prime} Q \Omega W-\int_{0}^{1} W^{\prime} \Omega^{\prime} Q_{1}^{\prime} Q^{-1} Q_{1} \Omega \int_{0}^{1} W\right\}^{-1}\left\{\int_{0}^{1} W^{\prime} \Omega^{\prime} Q_{1} \Omega W-\int_{0}^{1} W^{\prime} \Omega^{\prime} Q_{1} \Omega \int_{0}^{1} W\right\},
$$

and

$$
\begin{aligned}
\bar{V}_{X}= & M \Omega\left[\int_{0}^{1} W W^{\prime}-\int_{0}^{1} W \int_{0}^{1} W^{\prime}\right] \Omega^{\prime} M^{\prime}+\bar{\alpha}^{2} M Q^{-1} Q_{1} \Omega \int_{0}^{1} W \int_{0}^{1} W^{\prime} \Omega^{\prime} Q_{1}^{\prime} Q^{\prime-1} M^{\prime} \\
& +\bar{\alpha}^{2} M_{11} \Omega \int_{0}^{1} W W^{\prime} \Omega^{\prime} M_{11}^{\prime}-2 \bar{\alpha} M_{1} \Omega\left[\int_{0}^{1} W W^{\prime}-\int_{0}^{1} W \int_{0}^{1} W^{\prime}\right] \Omega^{\prime} M_{1}^{\prime} \\
& -\bar{\alpha}^{2} M_{1} Q^{-1} Q_{1} \Omega \int_{0}^{1} W \int_{0}^{1} W^{\prime} \Omega^{\prime} M_{1}^{\prime}-\bar{\alpha}^{2} M_{1} \Omega \int_{0}^{1} W \int_{0}^{1} W^{\prime} \Omega^{\prime} Q_{1}^{\prime} Q^{\prime-1} M_{1}^{\prime} .
\end{aligned}
$$

Also,

$$
T^{-1} S W \Rightarrow \frac{S S R^{r *}-S S R^{*}(\bar{\lambda})}{S S R^{*}(\bar{\lambda})}=O_{p}(1)
$$

uniformly in $\|\Omega\|$, where $\bar{\lambda}$ is the value of $\lambda$ that minimizes $S S R^{*}(\lambda)$ over the set $[\epsilon, 1-\epsilon]$,

$$
\begin{aligned}
S S R^{r *} \equiv & \left(1+\bar{\alpha}^{2}\right) \int_{0}^{1} W^{\prime} \Omega^{\prime} Q \Omega W-2 \bar{\alpha} \int_{0}^{1} W^{\prime} \Omega^{\prime} Q_{1} \Omega W \\
& -\int_{0}^{1} W^{\prime} \Omega^{\prime}\left[Q-\bar{\alpha} Q_{1}\right]^{\prime} Q^{-1}\left[Q-\bar{\alpha} Q_{1}\right] \Omega \int_{0}^{1} W
\end{aligned}
$$


and

$$
\begin{aligned}
S S R^{*}(\lambda) \equiv & \left(1+\bar{\alpha}_{u}^{2}\right) \int_{0}^{1} W^{\prime} \Omega^{\prime} Q \Omega W-2 \bar{\alpha}_{u} \int_{0}^{1} W^{\prime} \Omega^{\prime} Q_{1} \Omega W \\
& -\frac{1}{\lambda} \int_{0}^{\lambda} W^{\prime} \Omega^{\prime}\left[Q-\bar{\alpha}_{u} Q_{1}\right]^{\prime} Q^{-1}\left[Q-\bar{\alpha}_{u} Q_{1}\right] \Omega \int_{0}^{\lambda} W \\
& -\frac{1}{1-\lambda} \int_{\lambda}^{1} W^{\prime} \Omega^{\prime}\left[Q-\bar{\alpha}_{u} Q_{1}\right]^{\prime} Q^{-1}\left[Q-\bar{\alpha}_{u} Q_{1}\right] \Omega \int_{\lambda}^{1} W,
\end{aligned}
$$

with

$$
\begin{aligned}
\bar{\alpha}_{u} \equiv & \left\{\int_{0}^{1} W^{\prime} \Omega^{\prime} Q \Omega-\frac{1}{\lambda} \int_{0}^{\lambda} W^{\prime} \Omega^{\prime} Q_{1}^{\prime} Q^{-1} Q_{1} \Omega \int_{0}^{\lambda} W-\frac{1}{1-\lambda} \int_{\lambda}^{1} W^{\prime} \Omega^{\prime} Q_{1}^{\prime} Q^{-1} Q_{1} \Omega \int_{\lambda}^{1} W\right\}^{-1} \\
& \times\left\{\int_{0}^{1} W^{\prime} \Omega^{\prime} Q_{1} \Omega W-\frac{1}{\lambda} \int_{0}^{\lambda} W^{\prime} \Omega^{\prime} Q_{1} \Omega \int_{0}^{\lambda} W-\frac{1}{1-\lambda} \int_{\lambda}^{1} W^{\prime} \Omega^{\prime} Q_{1} \Omega \int_{\lambda}^{1} W\right\} .
\end{aligned}
$$

and $\bar{\alpha}$ as defined above.

The above expressions are quite complex. Consider the mean shift model with $X_{t}=\{1\}$ for all $t$. Then,

$$
T^{-1} q \hat{L} L \Rightarrow \frac{c^{2} \Omega^{2} \int_{0}^{1} W_{c}^{2}-c \Omega^{2}-2 c \sigma_{e}^{2}-\left(2 c \Omega^{2} /\left(1-e^{-2 c}\right)\right)\left[W_{c}(1)-c \int_{0}^{1} e^{-c r} W_{c}\right]^{2}}{\Omega^{2}+2 \sigma_{e}^{2}}=O_{p}(1)
$$

and

$$
\begin{aligned}
T^{-1} S W \Rightarrow & \left\{-2\left(\bar{\alpha}-\bar{\alpha}_{u}\right)\left[\frac{1}{2} \Omega^{2}(W(1)-1)-\sigma_{e}^{2}\right]+\Omega^{2} W(1)^{2}-\Omega^{2} W(\lambda)^{2}-\Omega^{2} W(1-\lambda)^{2}\right. \\
& \left.-\bar{\alpha}^{2} \Omega^{2} \int_{0}^{1} W^{2}+\bar{\alpha}_{u}^{2} \frac{1}{\lambda} \Omega^{2}\left(\int_{0}^{\lambda} W\right)^{2}+\bar{\alpha}_{u}^{2} \frac{1}{1-\lambda} \Omega^{2}\left(\int_{\lambda}^{1} W\right)^{2}\right\} /\left(\Omega^{2}+2 \sigma_{e}^{2}\right)=O_{p}(1),
\end{aligned}
$$

where

$$
\bar{\alpha} \equiv\left[(1 / 2)\left(W(1)^{2}-1\right)-\sigma_{e}^{2} / \Omega^{2}-W(1) \int_{0}^{1} W\right] /\left[\int_{0}^{1} W^{2}-\left(\int_{0}^{1} W\right)^{2}\right]
$$

and

$$
\bar{\alpha}_{u} \equiv \frac{(1 / 2)\left(W(1)^{2}-1\right)-\sigma_{e}^{2} / \Omega^{2}-W(\lambda)(1 / \lambda) \int_{0}^{\lambda} W-W(1-\lambda) \int_{\lambda}^{1} W /(1-\lambda)}{\int_{0}^{1} W^{2}-(1 / \lambda)\left(\int_{0}^{\lambda} W\right)^{2}-\left(\int_{\lambda}^{1} W\right)^{2} /(1-\lambda)}
$$

We again simulated these limit distributions for the DGP specified by (5). The means, as a function of $\Omega$, are presented in the first panel of Figure 7 along with the critical values pertaining to a $5 \%$ test and $T=100$. The results predict that on average the $q \hat{L} L$ test will not reject while the sup-Wald will. The second and third panels present the cdfs of the limit random variables for $\Omega=1$ and $\Omega=15$. The results clearly show that the $q \hat{L} L$ test has again a near-zero probability of rejecting and that this probability is not affected by the value of $\Omega$, while the sup-Wald has a large probability of rejecting for both values. These theoretical predictions are in accordance with the simulations discussed above. 


\subsection{Infrequent Breaks Model}

We now consider a model that specifies relatively rare occurrences of shifts in the parameters that is suitable for Models B and C. The specification is as follows:

$$
\beta_{t}=\sum_{j=1}^{t} \delta_{T, j}, \delta_{T, t}=\pi_{T, t} \eta_{t}
$$

Here, $\eta_{t} \sim$ i.i.d. $(0, \Omega)$ and $\pi_{T, t}$ is a binomial variable that takes value 1 with probability $p / T$ and value 0 with probability $(1-p / T)$, i.e. $\pi_{T, t} \sim$ i.i.d. $B(p / T, 1)$. We also assume that the components $\pi_{T, t}$ and $\eta_{t}$ are mutually independent. Note that the parameter $p$ is independent of the sample size $T$. Hence, as $T$ increases, the level shifts become relatively rare. This is an important ingredient that will allow us to derive interesting results. Intuitively, we need this specification to model structural changes, i.e., relatively infrequent events that affect the time series properties of the parameters in a permanent fashion. If $p / T$ converges to some value in $(0,1)$, the model is best construed as depicting a standard unit root process. This model is the most interesting to analyze since it bridges the gap between the two extremes of random walk parameter variations and a single change at some known date. Also, it does not suffer from the discontinuity problem present in the random walk model. Finally, it is more in line with the type of processes for which the $q \hat{L} L$ is especially designed.

A crucial ingredient used here is a Functional Central Limit Theorem for the cumulative level shifts process $u_{T, t}$. This has been considered by Georgiev (2002) and Leipus and Viano (2003). The results relevant to our analysis are stated in the following lemma.

Lemma 1 (Georgiev, 2002; Leipus and Viano, 2003) Consider $\beta_{t}$ specified by (12) with $0<p<\infty$ and define $u_{T}(s)=\sum_{t=1}^{[T s]} \delta_{T, t}$, then $u_{T}(s) \Rightarrow \Omega J(s)$, where $J(s)$ is a compound Poisson process defined by $J(s)=\sum_{j=0}^{N(s)} \eta_{j}^{*}$ with $N(s)$ being a Poisson process with jump intensity $p$, independent of $\eta_{j}^{*} \sim$ i.i.d. $\left(0, I_{k}\right)$ for all $j$.

We have the following theorem for the three scenarios considered.

Theorem 4 a) Assume that the $q \hat{L} L$ and sup-Wald (SW) test statistics are constructed from the static regression (5) with no correction for serial correlation and that the process is generated by (1) with $\beta_{t}$ specified by the random level shift model (12), then

$$
T^{-1} q \hat{L} L \Rightarrow \operatorname{tr}\left[\bar{V}_{X}^{-1 / 2} Q \Omega \Psi \Omega^{\prime} Q^{\prime} \bar{V}_{X}^{\prime-1 / 2}\right]=O_{p}(1)
$$


uniformly in $\|\Omega\|$, where $\Psi$ is a function of $J(r)$ defined by (A.14) in the appendix and

$$
\bar{V}_{X} \equiv \sigma_{e}^{2} Q+M \Omega\left[\int_{0}^{1} J J^{\prime}-\int_{0}^{1} J \int_{0}^{1} J^{\prime}\right] \Omega^{\prime} M^{\prime} .
$$

Also,

$$
T^{-1} S W \Rightarrow \frac{S S R^{r *}-S S R^{*}(\bar{\lambda})}{S S R^{*}(\bar{\lambda})}=O_{p}(1)
$$

where

$$
\begin{aligned}
S S R^{r *} & \equiv \sigma_{e}^{2}+\int_{0}^{1} J^{\prime} \Omega^{\prime} Q \Omega J-\int_{0}^{1} J^{\prime} \Omega^{\prime} Q \Omega \int_{0}^{1} J \\
S S R^{*}(\lambda) & \equiv \sigma_{e}^{2}+\int_{0}^{1} J^{\prime} \Omega^{\prime} Q \Omega J-\frac{1}{\lambda} \int_{0}^{\lambda} J^{\prime} \Omega^{\prime} Q \Omega \int_{0}^{\lambda} J-\frac{1}{1-\lambda} \int_{\lambda}^{1} J^{\prime} \Omega^{\prime} Q \Omega \int_{\lambda}^{1} J,
\end{aligned}
$$

and $\bar{\lambda}$ is the value of $\lambda$ that minimizes $S S R^{*}(\lambda)$ over the set $[\epsilon, 1-\epsilon]$.

We again considered simulating the limit random variables to assess the relative efficiency of the two tests for the DGP used in Section 4. The results are presented in Figure 8, which reports the means and cdfs of the limit random variables pertaining to the $q \hat{L} L$ and sup-Wald tests for $p / T=0.05,0.10$, and 0.50 (along with the critical values corresponding to a $5 \%$ size test with $T=100$ ). Looking at the cdfs of the limit distributions, the sup-Wald and $q \hat{L} L$ are nearly as powerful for small alternatives $(\Omega=1)$. On the other hand, the sup-Wald dominates the $q \hat{L} L$ for large alternatives. For example, the theoretical approximations to the power when $T=100, \Omega=1$ and $p / T=0.05,0.10$, and 0.50 are .703, .860 and .997, respectively, for the $q \hat{L} L$, and $.515, .690$ and .935 for the sup-Wald, so that the theory indeed predicts that the $q \hat{L} L$ is more powerful in the static case when the break is small. However, the power becomes quickly the same as $\Omega$ increases. What transpires from these results is the fact that both tests will perform well in the static case, with good power that is monotonic as documented in the simulations.

Consider now the case where a correction for serial correlation is applied. We then have the following results.

Theorem 5 Assume that the $q \hat{L} L$ and sup-Wald $(S W)$ tests are constructed from the static regression (5) and that the process is generated by (1) with $\beta_{t}$ specified by the random level shift model (12). Suppose serial correlation in the errors is accounted for using an estimate of the form (11) with the Bartlett kernel and the bandwidth chosen by Andrew's (1991) data dependent method based on an $A R(1)$ approximation. a) If $\left\{M-M_{11}\right\}_{i i}=0$ for some $i$, where $\{Z\}_{i i}$ is the ith diagonal element of the matrix $Z$, then

$$
T^{-2 / 3} q \hat{L} L \Rightarrow \operatorname{tr}\left[\bar{h}(0)^{-1 / 2} Q \Omega \Xi \Omega^{\prime} Q^{\prime} \bar{h}(0)^{\prime-1 / 2}\right]=O_{p}\left(\|\Omega\|^{-4 / 3}\right)
$$


uniformly in $\|\Omega\|$, where $\bar{h}(0) \equiv \bar{\Gamma}_{0}+\lim _{T \rightarrow \infty} T^{-1 / 3} \sum_{j=-T}^{T} \kappa\left(j, m_{T}\right)\left[\bar{\Gamma}_{j}+\bar{\Gamma}_{j}^{\prime}\right]$ with $\bar{\Gamma}_{j} \equiv$ $M_{j j} \Omega\left[\int_{0}^{1} J J^{\prime}-\int_{0}^{1} J \int_{0}^{1} J^{\prime}\right] \Omega^{\prime} M_{j j}^{\prime}, m \equiv 1.1447(\bar{\alpha} T)^{1 / 3}$,

$$
\begin{gathered}
\bar{\alpha} \equiv \sum_{i=1}^{k} \frac{4 \bar{\rho}_{i}^{2} \bar{\sigma}_{v i}^{4}}{\left(1-\bar{\rho}_{i}\right)^{6}\left(1+\bar{\rho}_{i}\right)^{2}} / \sum_{i=1}^{k} \frac{\bar{\sigma}_{v i}^{4}}{\left(1-\bar{\rho}_{i}\right)^{4}}, \\
\bar{\rho}_{i} \equiv \frac{\left\{M_{11}\right\}_{i i}^{2}\{\Omega\}_{i i}^{2}\left[\int_{0}^{1} J_{i}^{2}-\left(\int_{0}^{1} J_{i}\right)^{2}\right]}{\sigma_{e}^{2}\{Q\}_{i i}+\{M\}_{i i}^{2}\{\Omega\}_{i i}^{2}\left[\int_{0}^{1} J_{i}^{2}-\left(\int_{0}^{1} J_{i}\right)^{2}\right]},
\end{gathered}
$$

$\bar{\sigma}_{v i}^{2}=p \lim T^{-1} \sum_{t=2}^{T}\left(\hat{v}_{i t}-\hat{\rho}_{i} \hat{v}_{i t-1}\right)^{2}$ and $J_{i}$ is the ith element of $J$. Also,

$$
\begin{aligned}
T^{-2 / 3} S W \Rightarrow & \left(\frac{1}{\lambda^{*}} \Omega \int_{0}^{\lambda^{*}} J-\frac{1}{1-\lambda^{*}} \Omega \int_{\lambda^{*}}^{1} J\right)^{\prime} Q^{\prime}\left[\bar{h}_{1}(0)+\bar{h}_{2}(0)\right]^{-1} Q \\
& \times\left(\frac{1}{\lambda^{*}} \Omega \int_{0}^{\lambda^{*}} J-\frac{1}{1-\lambda^{*}} \Omega \int_{\lambda *}^{1} J\right)=O_{p}\left(\|\Omega\|^{-4 / 3}\right)
\end{aligned}
$$

uniformly in $\|\Omega\|$, where $\lambda^{*}$ is the value of $\lambda$ that maximizes the limit of the Wald test over the set $[\epsilon, 1-\epsilon], \bar{h}_{l}(0) \equiv \bar{\Gamma}_{l, 0}+\lim _{T \rightarrow \infty} T^{-1 / 3} \sum_{j=1}^{T} \kappa\left(j, m_{T}\right)\left[\bar{\Gamma}_{l, j}+\bar{\Gamma}_{l, j}^{\prime}\right] \quad(l=1,2)$ with

$$
\begin{aligned}
& \bar{\Gamma}_{1, j} \equiv M_{j j} \Omega\left[\int_{0}^{\lambda^{*}} J J^{\prime}-\int_{0}^{\lambda^{*}} J \int_{0}^{\lambda^{*}} J^{\prime}\right] \Omega^{\prime} M_{j j}^{\prime}, \\
& \bar{\Gamma}_{2, j} \equiv M_{j j} \Omega\left[\int_{\lambda^{*}}^{1} J J^{\prime}-\int_{\lambda^{*}}^{1} J \int_{\lambda^{*}}^{1} J^{\prime}\right] \Omega^{\prime} M_{j j}^{\prime},
\end{aligned}
$$

Also, $m_{l} \equiv 1.1447\left(\bar{\alpha}_{l} T\right)^{1 / 3}$, where

$$
\begin{aligned}
\bar{\alpha}_{l} & \equiv \sum_{i=1}^{k} \frac{4 \bar{\rho}_{l, i}^{2} \bar{\sigma}_{v i, l}^{4}}{\left(1-\bar{\rho}_{l, i}\right)^{6}\left(1+\bar{\rho}_{l, i}\right)^{2}} / \sum_{i=1}^{k} \frac{\bar{\sigma}_{v i, l}^{4}}{\left(1-\bar{\rho}_{l, i}\right)^{4}}, \\
\bar{\rho}_{1, i} & \equiv \frac{\left\{M_{11}\right\}_{i i}^{2}\{\Omega\}_{i i}^{2}\left[\left[\int_{0}^{\lambda^{*}} J_{i}^{2}-\left(\int_{0}^{\lambda^{*}} J_{i}\right)^{2}\right]\right.}{\sigma_{e}^{2}\{Q\}_{i i}+\{M\}_{i i}^{2}\{\Omega\}_{i i}^{2}\left[\left[\int_{0}^{\lambda^{*}} J_{i}^{2}-\left(\int_{0}^{\lambda^{*}} J_{i}\right)^{2}\right]\right.}, \\
\bar{\rho}_{2, i} & \equiv \frac{\left\{M_{11}\right\}_{i i}^{2}\{\Omega\}_{i i}^{2}\left[\int_{\lambda^{*}}^{1} J_{i}^{2}-\left(\int_{\lambda^{*}}^{1} J_{i}\right)^{2}\right]}{\sigma_{e}^{2}\{Q\}_{i i}+\{M\}_{i i}^{2}\{\Omega\}_{i i}^{2}\left[\int_{\lambda^{*}}^{1} J_{i}^{2}-\left(\int_{\lambda^{*}}^{1} J_{i}\right)^{2}\right]},
\end{aligned}
$$

$\bar{\sigma}_{v i, 1}^{2}=p \lim T^{-1} \sum_{t=2}^{T \lambda^{*}}\left(\hat{v}_{i t}-\hat{\rho}_{1, i} \hat{v}_{i t-1}\right)^{2}$ and $\bar{\sigma}_{v i, 2}^{2}=p \lim T^{-1} \sum_{t=T \lambda^{*}+1}^{T}\left(\hat{v}_{i t}-\hat{\rho}_{2, i} \hat{v}_{i t-1}\right)^{2}$. b) If $\left\{M-M_{11}\right\}_{i i} \neq 0$ for all $i$, then

$$
T^{-2 / 3} q \hat{L} L \Rightarrow \operatorname{tr}\left[\bar{h}(0)^{-1 / 2} Q \Omega \Xi \Omega^{\prime} Q^{\prime} \bar{h}(0)^{\prime-1 / 2}\right]=O_{p}(1)
$$

uniformly in $\|\Omega\|$, where $\bar{h}(0)$ and $\Xi$ are as defined in part (a) with

$$
\bar{\rho}_{i} \equiv \frac{\left\{M_{11}\right\}_{i i}^{2}\{\Omega\}_{i i}^{2}\left[\int_{0}^{1} J_{i}^{2}-\left(\int_{0}^{1} J_{i}\right)^{2}\right]}{\sigma_{e}^{2}\{Q\}_{i i}+\{M\}_{i i}^{2}\{\Omega\}_{i i}^{2}\left[\int_{0}^{1} J_{i}^{2}-\left(\int_{0}^{1} J_{i}\right)^{2}\right]} .
$$


Also,

$$
\begin{aligned}
T^{-2 / 3} S W \Rightarrow & \left(\frac{1}{\lambda^{*}} \Omega \int_{0}^{\lambda^{*}} J-\frac{1}{1-\lambda^{*}} \Omega \int_{\lambda^{*}}^{1} J\right)^{\prime} Q^{\prime}\left[\bar{h}_{1}(0)+\bar{h}_{2}(0)\right]^{-1} Q \\
& \times\left(\frac{1}{\lambda^{*}} \Omega \int_{0}^{\lambda^{*}} J-\frac{1}{1-\lambda^{*}} \Omega \int_{\lambda *}^{1} J\right)=O_{p}(1)
\end{aligned}
$$

uniformly in $\|\Omega\|$, where $\lambda^{*}$ is the value of $\lambda$ that maximizes the limit of the Wald test over the set $[\epsilon, 1-\epsilon]$ and $\bar{h}_{l}(0)$ is as defined in part (a) with

$$
\bar{\rho}_{1, i} \equiv \frac{\left\{M_{11}\right\}_{i i}^{2}\{\Omega\}_{i i}^{2}\left[\int_{0}^{\lambda^{*}} J_{i}^{2}-\left(\int_{0}^{\lambda^{*}} J_{i}\right)^{2}\right]}{\sigma_{e}^{2}\{Q\}_{i i}+\{M\}_{i i}^{2}\{\Omega\}_{i i}^{2}\left[\int_{0}^{\lambda^{*}} J_{i}^{2}-\left(\int_{0}^{\lambda^{*}} J_{i}\right)^{2}\right]},
$$

and

$$
\bar{\rho}_{2, i} \equiv \frac{\left\{M_{11}\right\}_{i i}^{2}\{\Omega\}_{i i}^{2}\left[\left[\int_{\lambda^{*}}^{1} J_{i}^{2}-\left(\int_{\lambda^{*}}^{1} J_{i}\right)^{2}\right]\right.}{\sigma_{e}^{2}\{Q\}_{i i}+\{M\}_{i i}^{2}\{\Omega\}_{i i}^{2}\left[\int_{\lambda^{*}}^{1} J_{i}^{2}-\left(\int_{\lambda^{*}}^{1} J_{i}\right)^{2}\right]} .
$$

The results show that when $\left\{M-M_{11}\right\}_{i i}=0$ for some $i$, as in the mean shift case, both tests will eventually exhibit zero power as the magnitude of the variance of the shocks increases since the limit values tend to zero (though for a fixed $\|\Omega\|$ both tests are consistent). Things are more interesting when $\left\{M-M_{11}\right\}_{i i} \neq 0$ for all $i$, as for the DGP used in the simulations presented in Section 4 . Here, both tests are again consistent but their scaled limit values are $O_{p}(1)$ uniformly in $\|\Omega\|$. The question of interest for assessing relative efficiency is how the limit distributions compare as a function of $\|\Omega\|$. This is too complex to do for the general case so we again present results based on simulating the limit distributions for the DGP considered in Section 4 (the method described in the previous section is used to approximate $\bar{h}(0))$. The results are presented in Figure 9, which report the means and cdfs of the limit random variables pertaining to the $q \hat{L} L$ and sup-Wald tests for $p / T=0.05,0.10$, and 0.50. Consider first the results for the $q \hat{L} L$ test. The mean of the limit distribution initially increases (in absolute value) as $\Omega$ increases but quickly drops down to a value below the critical value associated with a 5\% test using a sample with 100 observations. This decrease is quicker and more pronounced as the intensity of the level shifts $p / T$ increases. Hence, the theory predicts that on average the $q \hat{L} L$ test will reject for small values of $\Omega$ but will not for larger values especially as the level shift intensity increases. Things are similar for the sup-Wald test but there is one important difference. As $\Omega$ increases, the mean of the limit random variable stabilizes to a value implying a rejection for all values of $\Omega$ that are not very small. We plotted the cdf for a small $(\Omega=1)$ and large $(\Omega=15)$ value of the variance of the shocks. The figures make clear that the probability of rejecting the null 
hypothesis with the $q \hat{L} L$ test is small and shrinks as $\Omega$ increases or as the intensity of the level shifts increases. On the other hand, the probability of rejecting with the sup-Wald test is nearly one in all cases.

The next theorem pertains to the case with a lagged dependent variable as a regressor.

Theorem 6 Assume that the $q \hat{L} L$ and sup-Wald $(S W)$ tests are constructed from a dynamic regression of the form (6) with a lagged dependent variable as a regressor and the process is generated by (1) with $\beta_{t}$ specified by the random level shift model (12), then

$$
T^{-1} q \hat{L} L \Rightarrow \operatorname{tr}\left[\bar{V}_{X}^{-1 / 2}\left[Q-\bar{\alpha} Q_{1}\right] \Omega \Psi \Omega^{\prime}\left[Q-\bar{\alpha} Q_{1}\right]^{\prime} \bar{V}_{X}^{\prime-1 / 2}\right]=O_{p}(1)
$$

uniformly in $\|\Omega\|$, where $\Psi$ is defined by (A.14) in the appendix,

$$
\bar{\alpha} \equiv\left\{\sigma_{e}^{2}+\left[\int_{0}^{1} J^{\prime} \Omega^{\prime} Q \Omega J\right]-\int_{0}^{1} J^{\prime} \Omega^{\prime} Q_{1}^{\prime} Q^{-1} Q_{1} \Omega \int_{0}^{1} J\right\}^{-1}\left\{\int_{0}^{1} J^{\prime} \Omega^{\prime} Q_{1} \Omega J-\int_{0}^{1} J^{\prime} \Omega^{\prime} Q_{1} \Omega \int_{0}^{1} J\right\},
$$

and

$$
\begin{aligned}
\bar{V}_{X}= & \left(1+\bar{\alpha}^{2}\right) \sigma_{e}^{2}+M \Omega\left[\int_{0}^{1} J J^{\prime}-\int_{0}^{1} J \int_{0}^{1} J^{\prime}\right] \Omega^{\prime} M^{\prime}+\bar{\alpha}^{2} M Q^{-1} Q_{1} \Omega \int_{0}^{1} J \int_{0}^{1} J^{\prime} \Omega^{\prime} Q_{1}^{\prime} Q^{\prime-1} M^{\prime} \\
& +\bar{\alpha}^{2} M_{11} \Omega \int_{0}^{1} J J^{\prime} \Omega^{\prime} M_{11}^{\prime}-2 \bar{\alpha} M_{1} \Omega\left[\int_{0}^{1} J J^{\prime}-\int_{0}^{1} J \int_{0}^{1} J^{\prime}\right] \Omega^{\prime} M_{1}^{\prime} \\
& -\bar{\alpha}^{2} M_{1} Q^{-1} Q_{1} \Omega \int_{0}^{1} J \int_{0}^{1} J^{\prime} \Omega^{\prime} M_{1}^{\prime}-\bar{\alpha}^{2} M_{1} \Omega \int_{0}^{1} J \int_{0}^{1} J^{\prime} \Omega^{\prime} Q_{1}^{\prime} Q^{\prime-1} M_{1}^{\prime} .
\end{aligned}
$$

Also,

$$
T^{-1} S W \Rightarrow \frac{S S R^{r *}-S S R^{*}(\bar{\lambda})}{S S R^{*}(\bar{\lambda})}=O_{p}(1),
$$

where $\bar{\lambda}$ is the value of $\lambda$ that minimizes $S S R^{*}(\lambda)$ over the set $[\epsilon, 1-\epsilon]$,

$$
\begin{aligned}
S S R^{r *} \equiv & \left(1+\bar{\alpha}^{2}\right) \sigma_{e}^{2}+\left(1+\bar{\alpha}^{2}\right) \int_{0}^{1} J^{\prime} \Omega^{\prime} Q \Omega J-2 \bar{\alpha} \int_{0}^{1} J^{\prime} \Omega^{\prime} Q_{1} \Omega J \\
& -\int_{0}^{1} J^{\prime} \Omega^{\prime}\left[Q-\bar{\alpha} Q_{1}\right]^{\prime} Q^{-1}\left[Q-\bar{\alpha} Q_{1}\right] \Omega \int_{0}^{1} J,
\end{aligned}
$$

and

$$
\begin{aligned}
S S R^{*}(\lambda) \equiv & \left(1+\bar{\alpha}_{u}^{2}\right) \sigma_{e}^{2}+\left(1+\bar{\alpha}_{u}^{2}\right) \int_{0}^{1} J^{\prime} \Omega^{\prime} Q \Omega J-2 \bar{\alpha}_{u} \int_{0}^{1} J^{\prime} \Omega^{\prime} Q_{1} \Omega J \\
& -\frac{1}{\lambda} \int_{0}^{\lambda} J^{\prime} \Omega^{\prime}\left[Q-\bar{\alpha}_{u} Q_{1}\right]^{\prime} Q^{-1}\left[Q-\bar{\alpha}_{u} Q_{1}\right] \Omega \int_{0}^{\lambda} J \\
& -\frac{1}{1-\lambda} \int_{\lambda}^{1} J^{\prime} \Omega^{\prime}\left[Q-\bar{\alpha}_{u} Q_{1}\right]^{\prime} Q^{-1}\left[Q-\bar{\alpha}_{u} Q_{1}\right] \Omega \int_{\lambda}^{1} J
\end{aligned}
$$

with

$$
\begin{aligned}
\bar{\alpha}_{u}= & \left\{\sigma_{e}^{2}+\int_{0}^{1} J^{\prime} \Omega^{\prime} Q \Omega J-\frac{1}{\lambda} \int_{0}^{\lambda} J^{\prime} \Omega Q_{1}^{\prime} Q^{-1} Q_{1} \Omega \int_{0}^{\lambda} J-\frac{1}{1-\lambda} \int_{\lambda}^{1} J^{\prime} \Omega Q_{1}^{\prime} Q^{-1} Q_{1} \Omega \int_{\lambda}^{1} J\right\}^{-1} \\
& \times\left\{\int_{0}^{1} J^{\prime} \Omega^{\prime} Q_{1} \Omega J-\frac{1}{\lambda} \int_{0}^{\lambda} J^{\prime} \Omega^{\prime} Q_{1} \Omega \int_{0}^{\lambda} J-\frac{1}{1-\lambda} \int_{\lambda}^{1} J^{\prime} \Omega^{\prime} Q_{1} \Omega \int_{\lambda}^{1} J\right\} .
\end{aligned}
$$


Again, the theoretical expressions are quite complex and do not lend themselves easily to allow a comparison of the tests under general conditions. So we present simulations of the means and cdfs of the limit random variables for the DGP used in the simulations of Section 4. The results are presented in Figure 10, again for $p / T=0.05,0.10$ and 0.50. The results are qualitatively similar to the case with no lagged dependent variable and a correction for serial correlation. The mean of the limit random variable of the $q \hat{L} L$ test statistic initially increases (in absolute value) with $\Omega$ but quickly starts decreasing as $\Omega$ increases and at a lower value of $\Omega$ than for the case with a correction for serial correlation. The decrease in the case of $p / T=0.50$ happens so quickly that on average, the $q \hat{L} L$ test does not reject for any value of $\Omega$. On the other hand, the mean of the limit value of the sup-Wald test does not decrease as $\Omega$ increases but stabilizes to a level that implies a clear rejection. This becomes more pronounced as the intensity of the level shifts $p / T$ decreases. The cdfs of the limit random variables are presented for $\Omega=1$ and $\Omega=15$. When $\Omega=1$ and $p / T$ is small, the values imply a lower probability of rejection for the $q \hat{L} L$ than for the sup-Wald. When $\Omega=1$ and $p / T=0.5$ or when $\Omega=15$, the cdf corresponding to the sup-Wald test is much further to the right of that of (minus) the $q \hat{L} L$ test so that the sup-Wald test has much higher power, in accordance with the simulations.

\subsection{Single break model}

We now consider the case where the parameters exhibit a single break of fixed magnitude at some fixed date:

$$
\beta_{t}=\theta I_{t>\left[T \lambda_{c}\right]},
$$

where $\theta$ is a vector of constants representing the magnitudes of the break, $I$ is the indicator function and $\lambda_{c} \in[0,1]$ represents the break fraction. The probability limits of the scaled statistics are stated in the following theorem for the three scenarios considered. We first consider the case where one imposes a priori the assumption that the errors are serially uncorrelated.

Theorem 7 Assume that the $q \hat{L} L$ and sup-Wald $(S W)$ tests are constructed from the static regression (5) with no correction for serial correlation and that the process is generated by (1) with $\beta_{t}$ specified by (13), then

$$
p \lim _{T \rightarrow \infty} T^{-1} q \hat{L} L=\operatorname{tr}\left[\xi \bar{V}_{X}^{-1 / 2} Q \theta \theta^{\prime} Q^{\prime} \bar{V}_{X}^{\prime-1 / 2}\right]=O(1)
$$

uniformly in $\|\theta\|$, where

$$
\bar{V}_{X} \equiv \sigma_{e}^{2} Q+\lambda_{c}\left(1-\lambda_{c}\right) M \theta \theta^{\prime} M^{\prime},
$$


and

$$
\begin{aligned}
\xi= & \lambda_{c}\left(\lambda_{c}-1\right)+\frac{1}{c}\left(1-\lambda_{c}+\frac{1}{2} \lambda_{c}^{2}\right)-\frac{1}{c}\left(1-\lambda_{c}\right)^{2} e^{-c} \\
& -\frac{1}{2 c}\left(1-\lambda_{c}\right)^{2} e^{-2 c}-\frac{1}{c}\left(1-\lambda_{c}\right) e^{-c \lambda_{c}}+\frac{1}{c}\left(1-\lambda_{c}\right) e^{c\left(\lambda_{c}-2\right)}-\frac{1}{2 c} e^{2 c\left(\lambda_{c}-1\right)} \\
& -\frac{1}{2 c\left(1-e^{-2 c}\right)}\left[\lambda_{c}-1+\left(1-\lambda_{c}\right) e^{-2 c}+e^{-c \lambda_{c}}-e^{c\left(\lambda_{c}-2\right)}\right]^{2} .
\end{aligned}
$$

Also,

$$
p \lim _{T \rightarrow \infty} T^{-1} S W=\frac{\lambda_{c}\left(1-\lambda_{c}\right)}{\sigma_{e}^{2}} \theta^{\prime} Q \theta=O\left(\|\theta\|^{2}\right)
$$

so that $S W$ dominates $q \hat{L} L$ for sufficiently large $\|\theta\|$.

The results in this case are qualitatively different from those of the other models in that the limit values are non-stochastic functions of $\|\theta\|$ and other parameters of the DGP. The results reveal that the sup-Wald test dominates the $q \hat{L} L$ test for large enough values of the magnitude of the shifts $\|\theta\|$, irrespective of the other features of the DGP (within the class considered). This is so because the limit value of the sup-Wald test is an increasing function of $\|\theta\|$ while the limit value of the $q \hat{L} L$ test is uniformly bounded in $\|\theta\|$. For most DGPs however, the limit values of both test statistics imply a rejection of the null hypothesis when $\|\theta\|$ is not too small. Nonetheless, it is interesting to compare the limit values as a function of the magnitude of change. We do so again for the DGP considered in Section 4. The results are presented in Figure 11 for $\theta$ ranging between 0 and 5. It turns out that the sup-Wald test is far more efficient than the $q \hat{L} L$ test unless the magnitude of change is very small (in which case, the probability of rejection is small). In particular, the limit value of the $q \hat{L} L$ test flattens out as $\theta$ increases while that of the sup-Wald test increases exponentially. The implication for the finite sample power of the two tests is that if a $5 \%$ test is used both tests will have good and monotonic power (as documented in the simulations), but if a (unrealistic) very small size was used the $q \hat{L} L$ would have lower power than the sup-Wald.

The next theorem covers the case where a non-parametric correction for serial correlation in the errors is applied.

Theorem 8 Assume that the $q \hat{L} L$ and sup-Wald $(S W)$ tests are constructed from the static regression (5) and that the process is generated by (1) with $\beta_{t}$ specified by (13). Suppose serial correlation in the errors is accounted for using an estimate of the form (11) with the Bartlett kernel and the bandwidth chosen by Andrew's (1991) data dependent method based 
on an $A R(1)$ approximation. a) If $\left\{M-M_{11}\right\}_{i i}=0$ for some $i$, then

$$
p \lim _{T \rightarrow \infty} T^{-1} q \hat{L} L=\operatorname{tr}\left[\xi \bar{h}(0)^{-1 / 2} Q \theta \theta^{\prime} Q^{\prime} \bar{h}(0)^{\prime-1 / 2}\right]=O\left(\|\theta\|^{-4 / 3}\right)
$$

uniformly in $\|\theta\|$, where $\bar{h}(0) \equiv \bar{\Gamma}_{0}+\lim _{T \rightarrow \infty} T^{-1 / 3} \sum_{j=1}^{T} \kappa(j, m)\left[\bar{\Gamma}_{j}+\bar{\Gamma}_{j}^{\prime}\right]$ with $m \equiv 1.1447(\bar{\alpha} T)^{1 / 3}$,

$$
\bar{\alpha} \equiv \sum_{i=1}^{k} \frac{4 \bar{\rho}_{i}^{2} \bar{\sigma}_{v i}^{4}}{\left(1-\bar{\rho}_{i}\right)^{6}\left(1+\bar{\rho}_{i}\right)^{2}} / \sum_{i=1}^{k} \frac{\bar{\sigma}_{v i}^{4}}{\left(1-\bar{\rho}_{i}\right)^{4}},
$$

$\bar{\Gamma}_{j} \equiv \lambda_{c}\left(1-\lambda_{c}\right) M_{j j} \theta \theta^{\prime} M_{j j}^{\prime}$

$$
\bar{\rho}_{i} \equiv \frac{\lambda_{c}\left(1-\lambda_{c}\right)\left\{M_{11}\right\}_{i i}^{2}\left\{\theta \theta^{\prime}\right\}_{i i}}{\sigma_{e}^{2}\{Q\}_{i i}+\lambda_{c}\left(1-\lambda_{c}\right)\{M\}_{i i}^{2}\left\{\theta \theta^{\prime}\right\}_{i i}},
$$

and $\bar{\sigma}_{v i}^{2}=p \lim T^{-1} \sum_{t=2}^{T}\left(\hat{v}_{i t}-\hat{\rho}_{i} \hat{v}_{i t-1}\right)^{2}$, b) If $\left\{M-M_{11}\right\}_{i i} \neq 0$ for all $i$, then

$$
p \lim _{T \rightarrow \infty} T^{-1} q \hat{L} L=\operatorname{tr}\left[\xi \bar{h}(0)^{-1 / 2} Q \theta \theta^{\prime} Q^{\prime} \bar{h}(0)^{\prime-1 / 2}\right]=O(1)
$$

uniformly in $\|\theta\|$, where $\bar{h}(0)$ is defined as above. For the sup-Wald test, since $\hat{\Gamma}_{j} \stackrel{p}{\rightarrow} 0$ for $j \neq 0$ because the test uses unrestricted residuals in the construction of $\hat{h}(0)$, the results stated in Theorem 7 continue to apply so that in both cases, $S W$ dominates $q \hat{L} L$ for sufficiently large $\|\theta\|$.

When $\left\{M-M_{11}\right\}_{i i}=0$ for some $i$, as is the case in the mean shift model, the limit of the sup-Wald test is an increasing function of $\|\theta\|$ while that of the $q \hat{L} L$ test is decreasing in $\|\theta\|$. Hence for $\|\theta\|$ large enough, the sup-Wald test dominates the $q \hat{L} L$ test. Things are different when $\left\{M-M_{11}\right\}_{i i} \neq 0$ for all $i$, as is the case in the DGP used in Section 4 with an $\mathrm{AR}(1)$ process as the regressor. Here, the limit of the sup-Wald test increases as $\|\theta\|$ increases while the limit of the $q \hat{L} L$ test is uniformly bounded in $\|\theta\|$. To get an idea of the implications of this, Figure 12 presents the limit values of the $q \hat{L} L$ and sup-Wald tests as functions of $\|\theta\|$ for the DGP of Section 4. It is clear that the limit value of the sup-Wald test increases rapidly with $\|\theta\|$. However, the limit value of the $q \hat{L} L$ test initially increases with $\|\theta\|$ but rapidly decreases to stabilize at a low value, implying non-rejection. Hence, the $q \hat{L} L$ test will only reject for small values of $\|\theta\|$ while the sup-Wald test will reject for any value except, of course, very small ones.

The last theorem covers the case with a lagged dependent variable as a regressor. 
Theorem 9 Assume that the $q \hat{L} L$ and sup-Wald $(S W)$ tests are constructed from a dynamic regression of the form (6) with a lagged dependent variable as a regressor and the process is generated by (1) with $\beta_{t}$ specified by (13). Then,

$$
p \lim _{T \rightarrow \infty} T^{-1} q \hat{L} L=\operatorname{tr}\left[\xi \bar{V}_{X}^{-1 / 2}\left[Q-\bar{\alpha} Q_{1}\right] \theta \theta^{\prime}\left[Q-\bar{\alpha} Q_{1}\right]^{\prime} \bar{V}_{X}^{\prime-1 / 2}\right]=O(1)
$$

uniformly in $\|\theta\|$, where $\xi$ is as defined in Theorem 7 ,

$$
\bar{\alpha} \equiv\left[\sigma_{e}^{2}+\left(1-\lambda_{c}\right) \theta^{\prime} Q \theta-\left(1-\lambda_{c}\right)^{2} \theta^{\prime} Q_{1} Q^{-1} Q_{1} \theta\right]^{-1}\left[\lambda_{c}\left(1-\lambda_{c}\right) \theta^{\prime} Q_{1} \theta\right]
$$

and

$$
\begin{aligned}
\bar{V}_{X} \equiv & \left(1+\bar{\alpha}^{2}\right) \sigma_{e}^{2} Q+\bar{\alpha}^{2}\left(1-\lambda_{c}\right) M_{11} \theta \theta^{\prime} M_{11}^{\prime} \\
& +\left(1-\lambda_{c}\right) M\left[\lambda_{c} \theta \theta^{\prime}+\bar{\alpha}^{2}\left(1-\lambda_{c}\right) Q^{-1} Q_{1} \theta \theta^{\prime} Q_{1}^{\prime} Q^{-1 \prime}\right] M^{\prime} \\
& -\bar{\alpha}\left(1-\lambda_{c}\right) M_{1}\left[2 \lambda_{c} \theta \theta^{\prime}+\bar{\alpha}\left(1-\lambda_{c}\right)\left[Q^{-1} Q_{1} \theta \theta^{\prime}+\theta \theta^{\prime} Q_{1}^{\prime} Q^{-1 \prime}\right]\right] M_{1}^{\prime}
\end{aligned}
$$

Also,

$$
p \lim _{T \rightarrow \infty} T^{-1} S W=\left(\frac{S S R^{r^{*}}-\sigma_{e}^{2}}{\sigma_{e}^{2}}\right)=O\left(\|\theta\|^{2}\right),
$$

where

$$
\begin{aligned}
S S R^{r *} \equiv & \left(1+\bar{\alpha}^{2}\right) \sigma_{e}^{2}+\left(1-\lambda_{c}\right)\left(1+\bar{\alpha}^{2}\right) \theta^{\prime} Q \theta-2\left(1-\lambda_{c}\right) \bar{\alpha} \theta^{\prime} Q_{1} \theta \\
& -\left(1-\lambda_{c}\right)^{2} \theta^{\prime}\left[Q-\bar{\alpha} Q_{1}\right]^{\prime} Q^{-1}\left[Q-\bar{\alpha} Q_{1}\right] \theta
\end{aligned}
$$

so that $S W$ dominates $q \hat{L} L$ for sufficiently large $\|\theta\|$.

Here the results are qualitatively similar to the case with no lagged dependent variable and a correction for serial correlation when $\left\{M-M_{11}\right\}_{i i} \neq 0$ for all $i$. Both statistics diverge at rate $T$ but the limit value of the $q \hat{L} L$ is uniformly bounded in $\|\theta\|$ while that of the supWald test is an increasing function of $\|\theta\|$. Hence, for large enough $\|\theta\|$, the sup-Wald test dominates. Though the theoretical expressions are complex, one can easily obtain numerical values. This is done again for the case of the DGP of Section 4. The limit values as functions of $\|\theta\|$ are plotted in Figure 13. Again, this function is rapidly increasing as $\|\theta\|$ increases for the sup-Wald test so that it rejects for all but very small values of $\|\theta\|$. On the other hand, while the limit value of the $q \hat{L} L$ initially increases with $\|\theta\|$, it quickly reverts back to stabilize at a small value implying non-rejection. Hence, the sup-Wald dominates the $q \hat{L} L$, even for relatively small values of $\|\theta\|$. 


\section{Conclusion}

The usual methodology used to analyze the power of structural change tests and suggest tests with "optimal" properties relies on a local asymptotic framework where the magnitude of change shrinks towards zero as the sample size increases. This approach was adopted by Andrews and Ploberger (1994) who considered optimal tests based on maximizing some weighted average of the local asymptotic power function. It was also the approach adopted by EM to devise so-called optimal tests for general parameter variations. Our simulations have shown that the $q \hat{L} L$ test, labelled as optimal under this criterion, has very poor finite sample properties. It offers gains over the sup-Wald test, which is not an optimal test under this criterion, that are trivial when the break is small and there is no evidence of serial correlation in the residuals of the regression. This minor gain is achieved at the expense of very large power losses when considering models with serially correlated errors or dynamic models including a lagged dependent variable. Our simulation results are corroborated by the use of an alternative asymptotic framwork whereby the break sizes are fixed. Under this criterion, the sup-Wald test dominates the $q \hat{L} L$ test, which in some cases has zero relative asymptotic efficiency (i.e., power goes to zero as the break size increases).

The basic (though incomplete) reason for this feature is, on the one hand, the need to estimate the long-run variance of the errors to properly scale the statistics. Since no break is directly modelled, one needs to estimate this long-run variance using residuals that are 'contaminated' by the shifts under the alternative. As the shift gets larger, the estimate of the scale gets inflated with a resulting loss in power. With a lagged dependent variable, the problem is present because the shifts induce a bias of the autoregressive coefficient towards one (Perron, 1989, 1990), making the changes appear as outliers. The sup-Wald is less prone to this problem given that by allowing one break, it mitigates the problems to a great

extent. Further improvements are possible with the UDmax test. Methods to overcome the non-monotonic power problem of so-called partial sums of residuals type tests have been suggested by Altissimo and Corradi (2003) and Juhl and Xiao (2009). They suggest using non-parametric or local averaging methods where the mean is estimated using data in a neighborhood of a particular data point. The resulting estimates and tests are, however, very sensitive to the bandwidth used. A large one leads to properly sized tests in finite samples but with low power, and a small bandwidth leads to better power but large size distortions. There is currently no reliable method to appropriately chose this parameter in the context of structural changes. Also, this approach has been proposed only in the context 
of a change in mean. No results are available when correcting for serial correlation with arbitrary regressors or when dealing with models with a lagged dependent variable.

Our research, in conjunction with other work, has several implications. First, it implies that, in the context of structural change tests, one should abandon the use of a local asymptotic framework where the breaks are local to zero in delivering optimality criteria. These type of frameworks do not yield useful predictions about the finite sample properties of tests. Second, as argued in Perron (2006), one should also abandon partial-sums type tests for which only a model restricted to satisfy the null hypothesis of no change is used. These include the CUSUM, LM and $q \hat{L} L$ tests, among many others. These tests are plagued by the problem of a non-monotonic power function such that the power of the test can go to zero as the magnitude of change increases. There is no available alternative other than attempting to model, as best as possible, the nature of the changes present in the data. The sup-Wald test does this to a first approximation by taking the largest break into account. The UDmax test of Bai and Perron (1998) improves upon the sup-Wald test by taking more breaks into account. It may be the case that these tests are prone to size-distortions under the null hypothesis. Nevertheless, this is not a reason to abandon them in favor of tests with poor power properties. On the contrary, our work and others show that research should be directed at alleviating such size distortion problems. For progress in this direction see, e.g., Diebold and Chen (1996), Hansen (2000), Kejriwal (2009) and Prodan (2008). 


\section{References}

Altissimo, F., Corradi, V. (2003). Strong rules for detecting the number of breaks in a time series. Journal of Econometrics 117:207-244.

Andrews, D. W. K. (1991). Heteroskedasticity and autocorrelation consistent covariance matrix estimation. Econometrica 59:817-858.

Andrews, D. W. K. (1993). Tests for parameter instability and structural change with unknown change point. Econometrica 61:821-856.

Andrews, D. W .K., Ploberger, W. (1994). Optimal tests when a nuisance parameter is present only under the alternative. Econometrica 62:1383-1414.

Andrews, D. W. K., Ploberger, W. (1995). Admissibility of the likelihood ratio test when a nuisance parameter is present only under the alternative. Annals of Statistics 23:1609-1629.

Bahadur, R. R. (1960). Stochastic comparison of tests. Annals of Mathematical Statistics 31:276-295.

Bai, J., Perron, P. (1998). Estimating and testing linear models with multiple structural changes. Econometrica 66:47-78.

Bai, J., Perron, P. (2003). Computation and analysis of multiple structural change models. Journal of Applied Econometrics 18:1-22.

Brown, R. L., Durbin, J., Evans, J. M. (1975). Techniques for testing the constancy of regression relationships over time. Journal of the Royal Statistical Society B 37:149-163.

Deng, A., Perron, P. (2008). A non-local perspective on the power properties of the CUSUM and CUSUM of square tests for structural change. Journal of Econometrics 142:212-240.

Diebold, F. X., Chen, C. (1996). Testing structural stability with endogenous breakpoints: a size comparison of analytic and bootstrap procedures. Journal of Econometrics 70:221-241.

Elliott, G., Müller, U. K. (2006). Efficient tests for general persistent time variation in regression coefficients. Review of Economic Studies 73:907-940.

Franzini, L., Harvey A. (1983). Testing for deterministic trend and seasonal components in time series models. Biometrika 70:673-682.

Garcia, R., Perron, P. (1996). An analysis of the real interest rate under regime shifts. Review of Economics and Statistics 78:111-125.

Gardner, L. A. (1969). On detecting changes in the mean of normal variates. The Annals of Mathematical Statistics 40:116-126.

Georgiev, I. (2002). Functional weak limit theory for rare outlying events. Unpublished manuscript, European University Institute. 
Giacomini, R., Rossi, B. (2006). How stable is the forecasting performance of the yield curve for output growth? Oxford Bulletin of Economics and Statistics 68:783-795.

Hansen, B. E. (2000). Testing for structural change in conditional models. Journal of Econometrics 97:93-115.

Juhl, T., Xiao, Z. (2009). Testing for changing mean with monotonic power. Journal of Econometrics 148:14-24.

Kim, D., Perron, P. (2009). Assessing the relative power of structural break tests using a framework based on the approximate Bahadur slope. Journal of Econometrics 149:26-51.

Kejriwal, M. (2009). Tests for a shift in mean with good size and monotonic power. Economics Letters 102:78-82.

Leipus. R., Viano, M. C. (2003). Long memory and stochastic trends. Statistics and Probability Letters 61:177-190.

Musso, A., Stracca, L., van Dijk, D. (2009). Instability and nonlinearity in the Euro area Phillips curve. International Journal of Central Banking 5:181-212.

Nelson, F. D., Savin, N. E. (1990). The danger of extrapolating asymptotic local power. Econometrica 58:977-981.

Newey, W. K., West, K. D. (1987). A simple, positive semi-definite, heteroskedasticity and autocorrelation consistent covariance matrix. Econometrica 55:703-708.

Nyblom, J. (1989). Testing for constancy of parameters over time. Journal of the American Statistical Association 84:223-230.

Perron, P. (1989). The great crash, the oil price shock and the unit root hypothesis. Econometrica 57:1361-1401.

Perron, P. (1990). Testing for a unit root in a time series with a changing mean. Journal of Business and Economic Statistics 8:153-162.

Perron, P. (2006). Dealing with Structural Breaks. In: Patterson, K., Mills, T. C., eds. Palgrave Handbook of Econometrics, Vol. 1: Econometric Theory. Basingstoke: Palgrave Macmillan, pp. 278-352.

Phillips, P. C. B. (1988). Regression theory for near-integrated time series. Econometrica 56:1021-1044.

Prodan, R. (2008). Potential pitfalls in determining multiple structural changes with an application to purchasing power parity. Journal of Business and Economic Statistics 26:5065.

Shively, T. (1988). An exact test for a stochastic coefficient in a time series regression model. Journal of Time Series Analysis 9:81-88.

Vogelsang, T. J. (1999). Sources of non-monotonic power when testing for a shift in a dynamic time series. Journal of Econometrics 88:283-299. 


\section{Appendix}

Preliminaries for Theorems 1-9: Let $\hat{u}_{t}=\hat{V}_{X}^{-1 / 2} X_{t} \hat{e}_{t}$ and $\hat{\eta}_{t}$ be a $k \times 1$ vector of residuals whose $\mathrm{i}^{\text {th }}$ component is the residual from a regression of $\left\{\hat{\omega}_{t, i}\right\}$ on $\left\{\bar{r}^{t}\right\}$, as defined in Step 5 . Then from the definition in Step 6 we have $q \hat{L} L=\operatorname{tr}(\hat{Q})$, where

$$
\begin{aligned}
\hat{Q} & =\bar{r} \sum_{t=1}^{T} \hat{\eta}_{t} \hat{\eta}_{t}^{\prime}-\sum_{t=1}^{T} \hat{u}_{t} \hat{u}_{t}^{\prime} \\
& =\bar{r} \sum_{t=1}^{T}\left[\hat{\omega}_{t}-\left(\sum_{t=1}^{T} \bar{r}^{2 t}\right)^{-1}\left(\sum_{t=1}^{T} \bar{r}^{t} \hat{\omega}_{t}\right) \bar{r}^{t}\right]\left[\hat{\omega}_{t}-\left(\sum_{t=1}^{T} \bar{r}^{2 t}\right)^{-1}\left(\sum_{t=1}^{T} \bar{r}^{t} \hat{\omega}_{t}\right) \bar{r}^{t}\right]^{\prime}-\sum_{t=1}^{T} \hat{u}_{t} \hat{u}_{t}^{\prime} \\
& =\sum_{t=1}^{T} \hat{\omega}_{t} \hat{\omega}_{t}^{\prime}-\sum_{t=1}^{T} \hat{u}_{t} \hat{u}_{t}^{\prime}-\frac{c}{T} \sum_{t=1}^{T} \hat{\omega}_{t} \hat{\omega}_{t}^{\prime}-\left(\sum_{t=1}^{T} \bar{r}^{2 t}\right)^{-1}\left(\sum_{t=1}^{T} \bar{r}^{t} \hat{\omega}_{t}\right)\left(\sum_{t=1}^{T} \bar{r}^{t} \hat{\omega}_{t}\right)^{\prime},
\end{aligned}
$$

with $\bar{r}=1-c / T$. Using the fact that $\hat{\omega}_{s}=\bar{r} \hat{\omega}_{s-1}+\hat{u}_{s}-\hat{u}_{s-1}$, we have

$$
\hat{\omega}_{s}=\hat{u}_{s}-\frac{c}{T} \sum_{j=1}^{s-1} \bar{r}^{j-1} \hat{u}_{s-j}
$$

for $s=2, \ldots, T$ and $\hat{\omega}_{1}=\hat{u}_{1}$. Summing from 1 through $t-1$ gives

$$
\sum_{s=1}^{t-1} \hat{\omega}_{s}=\sum_{s=1}^{t-1} \bar{r}^{s-1} \hat{u}_{t-s}
$$

We can then show that the first two terms of (A.1) are given by

$$
\begin{aligned}
\sum_{t=1}^{T} \hat{\omega}_{t} \hat{\omega}_{t}^{\prime}-\sum_{t=1}^{T} \hat{u}_{t} \hat{u}_{t}^{\prime} & =\frac{c^{2}}{T^{2}} \sum_{t=1}^{T}\left[\sum_{s=1}^{t-1} \bar{r}^{s-1} \hat{u}_{t-s}\right]\left[\sum_{s=1}^{t-1} \bar{r}^{s-1} \hat{u}_{t-s}\right]^{\prime} \\
& -\frac{c}{T} \sum_{t=1}^{T} \hat{u}_{t}\left[\sum_{s=1}^{t-1} \bar{r}^{s-1} \hat{u}_{t-s}\right]^{\prime}-\frac{c}{T} \sum_{t=1}^{T}\left[\sum_{s=1}^{t-1} \bar{r}^{s-1} \hat{u}_{t-s}\right] \hat{u}_{t}^{\prime}
\end{aligned}
$$

For the fourth term of (A.1), we have

$$
\sum_{t=1}^{T} \bar{r}^{t} \hat{\omega}_{t}=\sum_{t=1}^{T} \bar{r}^{t} \hat{u}_{t}-\frac{c}{T} \sum_{t=1}^{T} \bar{r}^{t} \sum_{s=1}^{t-1} \bar{r}^{s-1} \hat{u}_{t-s}
$$

Since $\hat{u}_{t}=\hat{V}_{X}^{-1 / 2} X_{t} \hat{e}_{t}$, it is useful to derive an expression in terms of $\hat{v}_{t}=X_{t} \hat{e}_{t}$, separating $\hat{V}_{X}$ from $\hat{u}_{t}$. By substituting (A.4) and (A.5) into (A.1) we obtain

$$
\begin{aligned}
\hat{Q} & =\hat{V}_{X}^{-1 / 2}\left[c^{2} A_{1, T}-c\left[A_{2, T}+A_{2, T}^{\prime}\right]-c A_{3, T}\right. \\
& \left.-A_{6, T}^{-1} \times\left[A_{4, T}-c A_{5, T}\right]\left[A_{4, T}-c A_{5, T}\right]^{\prime}\right] \hat{V}_{X}^{\prime-1 / 2}+\tau_{t},
\end{aligned}
$$

where $\tau_{t}$ is of lower order than the other terms uniformly in $\|\Omega\|, \hat{V}_{X}=T^{-1} \sum_{t=1}^{T} \hat{v}_{t} \hat{v}_{t}^{\prime}$, $A_{1, T}=T^{-2} \sum_{t=1}^{T}\left[\sum_{s=1}^{t-1} \bar{r}^{s-1} \hat{v}_{t-s}\right]\left[\sum_{s=1}^{t-1} \bar{r}^{s-1} \hat{v}_{t-s}\right]^{\prime}, A_{2, T}=T^{-1} \sum_{t=1}^{T} \hat{v}_{t}\left[\sum_{s=1}^{t-1} \bar{r}^{s-1} \hat{v}_{t-s}\right]^{\prime}, A_{3, T}=$ 
$T^{-1} \sum_{t=1}^{T} \hat{v}_{t} \hat{v}_{t}^{\prime}, A_{4, T}=T^{-1 / 2} \sum_{t=1}^{T} \bar{r}^{t} \hat{v}_{t}, A_{5, T}=T^{-3 / 2} \sum_{t=1}^{T} \bar{r}^{t} \sum_{s=1}^{t-1} \bar{r}^{s-1} \hat{v}_{t-s}$, and $A_{6, T}=$ $T^{-1} \sum_{t=1}^{T} \bar{r}^{2 t} \stackrel{p}{\rightarrow}(1 / 2 c)\left[1-e^{-2 c}\right]$. Our task is then to derive the limits of $\hat{V}_{X}$ and $A_{i, T}$, $i=1, \ldots, 5$.

For the proofs of Theorems 2-4 we shall use the following Lemma, whose results are by now standard (e.g., Phillips, 1988).

Lemma A.1 Let $y_{t}=\sum_{s=1}^{t}(1-(c / T))^{t-s} \varepsilon_{s}$ and assume that $\varepsilon_{s}$ is uniform mixing with mixing coefficient $\phi$ of size $-r /(2 r-2)$ or strong mixing with mixing coefficient $\alpha$ of size $-r /(r-2), r>2$. Suppose further that $E\left|\varepsilon_{s}\right|<\Delta<\infty$ and $E\left(\varepsilon_{s}\right)=0$ for $s=1,2 \ldots$ If $\left\{\varepsilon_{s}\right\}$ is globally covariance stationary with nonsingular global covariance matrix $\Omega=$ $\lim _{T \rightarrow \infty} \operatorname{var}\left(T^{-1 / 2} \sum_{t=1}^{T} \varepsilon_{t}\right)$, then a) $\left.T^{-1 / 2} y_{[T r]} \Rightarrow \Omega W_{c}(r) ; b\right) T^{-3 / 2} \sum_{t=1}^{T} y_{t} \Rightarrow \Omega \int_{0}^{1} W_{c}(r) d r$; c) $T^{-2} \sum_{t=1}^{T} y_{t} y_{t}^{\prime} \Rightarrow \Omega \int_{0}^{1} W_{c}(r) W_{c}(r)^{\prime} d r \Omega$; and d) $T^{-1} \sum_{t=1}^{[T r]}(1-(c / T))^{s} \sum_{t=1}^{[T r]-s} \varepsilon_{t} \Rightarrow$ $(1 / c) \Omega\left[W(r)-W_{c}(r)\right]$, where $W_{c}(r)=W(r)-c \int_{0}^{r} e^{-c \varsigma} W(r-\varsigma) d \varsigma$.

Proof of Theorem 1 (random walk parameters, i.i.d. errors assumed): Under $H_{0}$, the OLS estimate of $\beta$ is $\hat{\beta}=\left[T^{-1} \sum_{t=1}^{T} X_{t} X_{t}^{\prime}\right]^{-1}\left[T^{-1} \sum_{t=1}^{T} X_{t} X_{t}^{\prime} \beta_{t}+T^{-1} \sum_{t=1}^{T} X_{t} e_{t}\right]$, so that $T^{-1 / 2} \hat{\beta} \Rightarrow \Omega \int_{0}^{1} W(r) d r$. The OLS residuals are given by

$$
\hat{e}_{t}=e_{t}+X_{t}^{\prime} \beta_{t}-X_{t}^{\prime} \hat{\beta}
$$

which implies that

$$
\hat{v}_{t}=X_{t} e_{t}+X_{t} X_{t}^{\prime} \beta_{t}-X_{t} X_{t}^{\prime} \hat{\beta} .
$$

Using these representations, we have the following limit results for the components of $\hat{Q}$ :

$$
\begin{aligned}
& T^{-1} \hat{V}_{X} \\
= & T^{-2} \sum_{t=1}^{T} \hat{v}_{t} \hat{v}_{t}^{\prime} \\
= & T^{-2} \sum_{t=1}^{T}\left\{X_{t} X_{t}^{\prime} \beta_{t} \beta_{t}^{\prime} X_{t} X_{t}^{\prime}-X_{t} X_{t}^{\prime} \beta_{t} \hat{\beta}^{\prime} X_{t} X_{t}^{\prime}-X_{t} X_{t}^{\prime} \hat{\beta} \beta_{t}^{\prime} X_{t} X_{t}^{\prime}+X_{t} X_{t}^{\prime} \hat{\beta} \hat{\beta}^{\prime} X_{t} X_{t}^{\prime}\right\}+o_{p}(1) \\
\Rightarrow & M \Omega\left[\int_{0}^{1} W W^{\prime}-\int_{0}^{1} W \int_{0}^{1} W^{\prime}\right] \Omega^{\prime} M^{\prime} \equiv \bar{V}_{X}=O_{p}\left(\|\Omega\|^{2}\right), \\
& T^{-2} A_{1, T} \\
= & T^{-4} \sum_{t=1}^{T}\left[\sum_{s=1}^{t-1} \bar{r}^{s-1} \hat{v}_{t-s}\right]\left[\sum_{s=1}^{t-1} \bar{r}^{s-1} \hat{v}_{t-s}\right]^{\prime} \\
= & T^{-4} \sum_{t=1}^{T}\left\{\left[\sum_{s=1}^{t-1} \bar{r}^{s-1} X_{t-s} X_{t-s}^{\prime} \beta_{t-s}\right]\left[\sum_{s=1}^{t-1} \bar{r}^{s-1} X_{t-s} X_{t-s}^{\prime} \beta_{t-s}\right]^{\prime}\right. \\
& -\left[\sum_{s=1}^{t-1} \bar{r}^{s-1} X_{t-s} X_{t-s}^{\prime} \beta_{t-s}\right]\left[\sum_{s=1}^{t-1} \bar{r}^{s-1} X_{t-s} X_{t-s}^{\prime} \hat{\beta}\right]^{\prime}-\left[\sum_{s=1}^{t-1} \bar{r}^{s-1} X_{t-s} X_{t-s}^{\prime} \hat{\beta}\right]\left[\sum_{s=1}^{t-1} \bar{r}^{s-1} X_{t-s} X_{t-s}^{\prime} \beta_{t-s}\right]^{\prime} \\
& \left.+\left[\sum_{s=1}^{t-1} \bar{r}^{s-1} X_{t-s} X_{t-s}^{\prime} \hat{\beta}\right]\left[\sum_{s=1}^{t-1} \bar{r}^{s-1} X_{t-s} X_{t-s}^{\prime} \hat{\beta}\right]^{\prime}\right\}+o_{p}(1)
\end{aligned}
$$




$$
\begin{aligned}
& \Rightarrow \frac{1}{c^{2}} Q \Omega\left[\int_{0}^{1}\left(W-W_{c}\right)\left(W-W_{c}\right)^{\prime}\right] \Omega^{\prime} Q^{\prime}-\frac{1}{c} Q \Omega \int_{0}^{1}\left(\int_{0}^{r} e^{-c \varsigma} d \varsigma\right)\left(W-W_{c}\right) d r \int_{0}^{1} W^{\prime} \Omega^{\prime} Q^{\prime} \\
& -\frac{1}{c} Q \Omega \int_{0}^{1} W\left[\int_{0}^{1}\left(\int_{0}^{r} e^{-c \varsigma} d \varsigma\right)\left(W-W_{c}\right) d r\right]^{\prime} \Omega^{\prime} Q^{\prime}+Q \Omega \int_{0}^{1}\left(\int_{0}^{r} e^{-c \varsigma} d \varsigma\right)^{2} d r \int_{0}^{1} W \int_{0}^{1} W^{\prime} \Omega^{\prime} Q^{\prime} \\
& =O_{p}\left(\|\Omega\|^{2}\right) \text {, } \\
& T^{-2} A_{2, T}=T^{-3} \sum_{t=1}^{T} \hat{v}_{t}\left[\sum_{s=1}^{t-1} \bar{r}^{s-1} \hat{v}_{t-s}\right]^{\prime} \\
& =T^{-3} \sum_{t=1}^{T}\left\{X_{t} X_{t}^{\prime} \beta_{t}\left[\sum_{s=1}^{t-1} \bar{r}^{s-1} X_{t-s} X_{t-s}^{\prime} \beta_{t-s}\right]^{\prime}-X_{t} X_{t}^{\prime} \beta_{t}\left[\sum_{s=1}^{t-1} \bar{r}^{s-1} X_{t-s} X_{t-s}^{\prime} \hat{\beta}\right]^{\prime}\right. \\
& \left.-X_{t} X_{t}^{\prime} \hat{\beta}\left[\sum_{s=1}^{t-1} \bar{r}^{s-1} X_{t-s} X_{t-s}^{\prime} \beta_{t-s}\right]^{\prime}+X_{t} X_{t}^{\prime} \hat{\beta}\left[\sum_{s=1}^{t-1} \bar{r}^{s-1} X_{t-s} X_{t-s}^{\prime} \hat{\beta}\right]^{\prime}\right\}+o_{p}(1) \\
& \Rightarrow \frac{1}{c} Q \Omega\left[\int_{0}^{1}\left(W W^{\prime}-W W_{c}^{\prime}\right)\right] \Omega^{\prime} Q^{\prime}-Q \Omega \int_{0}^{1}\left(\int_{0}^{r} e^{-c \varsigma} d \varsigma\right) W d r \int_{0}^{1} W^{\prime} \Omega^{\prime} Q^{\prime} \\
& -\frac{1}{c} Q \Omega \int_{0}^{1} W\left[\int_{0}^{1}\left(W-W_{c}\right)\right]^{\prime} \Omega^{\prime} Q^{\prime}+Q \Omega \int_{0}^{1} \int_{0}^{r} e^{-c \varsigma} d \varsigma d r \int_{0}^{1} W \int_{0}^{1} W^{\prime} \Omega^{\prime} Q^{\prime}=O_{p}\left(\|\Omega\|^{2}\right), \\
& T^{-2} A_{3, T} \rightarrow 0_{k \times k}, \\
& T^{-1} A_{4, T}=T^{-3 / 2} \sum_{t=1}^{T} \bar{r}^{t} \hat{v}_{t}=T^{-3 / 2} \sum_{t=1}^{T} \bar{r}^{t} X_{t} X_{t}^{\prime} \beta_{t}-T^{-3 / 2} \sum_{t=1}^{T} \bar{r}^{t} X_{t} X_{t}^{\prime} \hat{\beta}+o_{p}(1) \\
& \Rightarrow Q \Omega \int_{0}^{1} e^{-c r} W-Q \Omega \int_{0}^{1} e^{-c r} \int_{0}^{1} W=O_{p}(\|\Omega\|), \\
& T^{-1} A_{5, T}=T^{-5 / 2} \sum_{t=1}^{T} \bar{r}^{t} \sum_{s=1}^{t-1} \bar{r}^{s-1} \hat{v}_{t-s} \\
& =T^{-5 / 2} \sum_{t=1}^{T} \bar{r}^{t} \sum_{s=1}^{t-1} \bar{r}^{s-1} X_{t-s} X_{t-s}^{\prime} \beta_{t-s}-T^{-5 / 2} \sum_{t=1}^{T} \bar{r}^{t} \sum_{s=1}^{t-1} \bar{r}^{s-1} X_{t-s} X_{t-s}^{\prime} \hat{\beta}+o_{p}(1) \\
& \Rightarrow \frac{1}{c} Q \Omega\left[\int_{0}^{1} e^{-c r}\left(W-W_{c}\right)\right]-Q \Omega \int_{0}^{1} e^{-c r} \int_{0}^{r} e^{-c \varsigma} d \varsigma d r \int_{0}^{1} W=O_{p}(\|\Omega\|) .
\end{aligned}
$$

Collecting terms, by (A.6) we have, $T^{-1} q \hat{L} L \Rightarrow \operatorname{tr}\left\{\bar{V}_{X}^{-1 / 2} Q \Omega \Xi \Omega^{\prime} Q^{\prime} \bar{V}_{X}^{\prime-1 / 2}\right\}$, where

$$
\Xi=\int_{0}^{1} W_{c} W_{c}^{\prime}-\int_{0}^{1} W W^{\prime}+\int_{0}^{1} W \int_{0}^{1} W^{\prime}-\frac{2 c}{1-e^{-2 c}} \int_{0}^{1} e^{-c r} W \int_{0}^{1} e^{-c r} W^{\prime} .
$$

We now consider the limit of the sup-Wald test. The unrestricted OLS residuals allowing for a single break at some $\lambda \in[0,1]$ are given by

$$
\hat{e}(\lambda)_{t}=e_{t}+X_{t}^{\prime} \beta_{t}-X_{1}(\lambda)_{t}^{\prime} \hat{\beta}_{1}-X_{2}(\lambda)_{t}^{\prime} \hat{\beta}_{2}
$$


where $\hat{\beta}_{1}$ and $\hat{\beta}_{2}$ are OLS estimates of $\beta_{1}$ and $\beta_{2}$. It is straightforward to show that $T^{-1 / 2} \hat{\beta}_{1} \Rightarrow$ $(1 / \lambda) \Omega \int_{0}^{\lambda} W$ and $T^{-1 / 2} \hat{\beta}_{2} \Rightarrow(1 /(1-\lambda)) \Omega \int_{\lambda}^{1} W$. Using (A.9), the unrestricted sum of squared residuals is such that

$$
\begin{aligned}
T^{-2} S S R_{T}(\lambda) & =T^{-2} \sum_{t=1}^{T}\left[e_{t}+X_{t}^{\prime} \beta_{t}-X_{1}(\lambda)_{t}^{\prime} \hat{\beta}_{1}-X_{2}(\lambda)_{t}^{\prime} \hat{\beta}_{2}\right]^{2} \\
& \Rightarrow\left[\int_{0}^{1} W^{\prime} \Omega^{\prime} Q \Omega W\right]-\frac{1}{\lambda} \int_{0}^{\lambda} W^{\prime} \Omega^{\prime} Q \Omega \int_{0}^{\lambda} W-\frac{1}{1-\lambda} \int_{\lambda}^{1} W^{\prime} \Omega^{\prime} Q \Omega \int_{\lambda}^{1} W \\
& \equiv S S R^{*}(\lambda)=O_{p}\left(\|\Omega\|^{2}\right),
\end{aligned}
$$

while using (A.7), the restricted sum of squared residuals is such that

$$
\begin{aligned}
T^{-2} S S R_{T}^{r} & =T^{-2} \sum_{t=1}^{T}\left[e_{t}+X_{t}^{\prime} \beta_{t}-X_{t}^{\prime} \hat{\beta}\right]^{2} \\
& \Rightarrow \int_{0}^{1} W^{\prime} \Omega^{\prime} Q \Omega W-\int_{0}^{1} W^{\prime} \Omega^{\prime} Q \Omega \int_{0}^{1} W \equiv S S R^{r *}=O_{p}\left(\|\Omega\|^{2}\right) .
\end{aligned}
$$

By the continuous mapping theorem, the limit of the sup-Wald test is given by

$$
T^{-1} S W=\left(\frac{T^{-2} S S R_{T}^{r}-T^{-2} S S R_{T}\left(\bar{\lambda}_{T}\right)}{T^{-2} S S R_{T}\left(\bar{\lambda}_{T}\right)}\right) \Rightarrow\left(\frac{S S R^{r *}-S S R^{*}(\bar{\lambda})}{S S R^{*}(\bar{\lambda})}\right)=O_{p}(1),
$$

where $\bar{\lambda}_{T}$ is the value of $\lambda$ that minimizes $S S R_{T}(\lambda)$ and $\bar{\lambda}$ minimizes $S S R^{*}(\lambda)$.

Proof of Theorem 2 (random walk parameters, correction for serial correlation): If we take into account serial correlation in the errors, we replace $\hat{V}_{X}$ with the heteroskedasticity and autocorrelation robust estimate given by

$$
\hat{h}(0)=\hat{V}_{X}+\sum_{j=1}^{T-1} \kappa(j, m)\left[\hat{\Gamma}_{j}+\hat{\Gamma}_{j}^{\prime}\right]
$$

where $\hat{\Gamma}_{j}=T^{-1} \sum_{t=j+1}^{T} \hat{v}_{t} \hat{v}_{t-j}^{\prime}$. For the $q \hat{L} L$ test, this is constructed by using $\hat{v}_{t}$ given in (A.8). We first consider the limit of $\hat{\Gamma}_{j}$. We have,

$$
\begin{aligned}
T^{-1} \hat{\Gamma}_{j}= & T^{-2} \sum_{t=j+1}^{T}\left\{X_{t} X_{t}^{\prime} \beta_{t} \beta_{t-j}^{\prime} X_{t-j} X_{t-j}^{\prime}-X_{t} X_{t}^{\prime} \beta_{t} \hat{\beta}^{\prime} X_{t-j} X_{t-j}^{\prime}\right. \\
& \left.-X_{t} X_{t}^{\prime} \hat{\beta} \beta_{t-j}^{\prime} X_{t-j} X_{t-j}^{\prime}+X_{t} X_{t}^{\prime} \hat{\beta} \hat{\beta}^{\prime} X_{t-j} X_{t-j}^{\prime}\right\}+o_{p}(1) \\
\Rightarrow & M_{j j} \Omega\left[\int_{0}^{1} W W^{\prime}-\int_{0}^{1} W \int_{0}^{1} W^{\prime}\right] \Omega^{\prime} M_{j j}^{\prime} \equiv \bar{\Gamma}_{j}=O_{p}\left(\|\Omega\|^{2}\right)
\end{aligned}
$$

uniformly in $j=o(T)$. In the next step, we use Andrews's (1991) method based on choosing the bandwidth such that, for the Bartlett kernel $m=1.1447(\alpha T)^{1 / 3}$, where

$$
\alpha=\sum_{i=1}^{k} \frac{4 \hat{\rho}_{i}^{2} \hat{\sigma}_{v i}^{4}}{\left(1-\hat{\rho}_{i}\right)^{6}\left(1+\hat{\rho}_{i}\right)^{2}} / \sum_{i=1}^{k} \frac{\hat{\sigma}_{v i}^{4}}{\left(1-\hat{\rho}_{i}\right)^{4}}
$$


with $\hat{\rho}_{i}$ and $\hat{\sigma}_{v i}$ being the OLS estimates in an $\mathrm{AR}(1)$ regression applied to the $i$ th variable of $\hat{v}_{t}$. We have

$$
\hat{\rho}_{i}=\frac{\left\{\hat{\Gamma}_{1}\right\}_{i i}}{\left\{\hat{\Gamma}_{0}\right\}_{i i}} \Rightarrow \frac{\left\{M_{11}\right\}_{i i}^{2}\{\Omega\}_{i i}^{2}\left[\int_{0}^{1} W_{i}^{2}-\left(\int_{0}^{1} W_{i}\right)^{2}\right]}{\{M\}_{i i}^{2}\{\Omega\}_{i i}^{2}\left[\int_{0}^{1} W_{i}^{2}-\left(\int_{0}^{1} W_{i}\right)^{2}\right]}=\frac{\left\{M_{11}\right\}_{i i}^{2}}{\{M\}_{i i}^{2}} .
$$

If $\left\{M-M_{11}\right\}_{i i}=0$, the limit value of $\hat{\rho}_{i}$ is one and

$$
T\left(\hat{\rho}_{i}-1\right) \Rightarrow \frac{-W_{i}(1)^{2}-\left(\int_{0}^{1} W_{i}\right)^{2}+W_{i}(1) \int_{0}^{1} W_{i}}{\int_{0}^{1} W_{i}^{2}-\left(\int_{0}^{1} W_{i}\right)^{2}} \equiv \phi_{i}=O_{p}(1) .
$$

Since $\hat{\sigma}_{v i}^{2}$ is the variance estimate of the differenced series and is bounded, the $i$ th component such that $\left\{M-M_{11}\right\}_{i i}=0$ dominates both the numerator and denominator of $\alpha$. Hence, $\alpha=$ $O_{p}\left(T^{2}\right)$ and $m=O_{p}\left(T^{2 / 3} T^{1 / 3}\right)=O_{p}(T)$. Therefore, $\hat{h}(0)=O_{p}\left(\|\Omega\|^{2} T^{2}\right)$ and substituting its limit value in $\bar{V}_{X}$ in the result of Theorem 1 gives the stated result. If $\left\{M-M_{11}\right\}_{i i} \neq 0$ for all $i$, then any $\hat{\rho}_{i}$ converges to a value less than one and $\hat{\sigma}_{v i}^{2}$ is the variance estimate of the AR1 filtered series whose coefficient estimate is less than one and is bounded. Hence, $\alpha=O_{p}(1)$ and $m=O_{p}\left(T^{1 / 3}\right)$. Therefore $\hat{h}(0)=O_{p}\left(\|\Omega\|^{2} T^{4 / 3}\right)$ giving the result in part(b) for the $q \hat{L} L$ test.

For the sup-Wald test, we use the expression

$$
\text { Wald }=\frac{1}{T}\left(\frac{T-2 k}{k}\right) \hat{\beta}^{\prime} H^{\prime}\left(H \hat{V} H^{\prime}\right)^{-1} H \hat{\beta},
$$

where $\hat{V} \equiv\left(\bar{X}^{\prime} \bar{X}\right)^{-1} \hat{V}_{X}\left(\bar{X}^{\prime} \bar{X}\right)^{-1}$. First, we have,

$$
T^{-1 / 2} \hat{\beta}^{\prime} H^{\prime}=T^{-1 / 2} \hat{\beta}_{1}-T^{-1 / 2} \hat{\beta}_{2} \Rightarrow \frac{1}{\lambda} \Omega \int_{0}^{\lambda} W-\frac{1}{1-\lambda} \Omega \int_{\lambda}^{1} W=O_{p}(1) .
$$

Second, $T^{-1 / 3} \hat{V}=\operatorname{diag}\left(\hat{V}_{1}, \hat{V}_{2}\right)$, where

$$
\begin{aligned}
\hat{V}_{1} & \equiv\left(T^{-1} \sum_{t=1}^{[T \lambda]} X_{t} X_{t}^{\prime}\right)^{-1}\left(T^{-4 / 3} \hat{h}_{1}(0)\right)\left(T^{-1} \sum_{t=1}^{[T \lambda]} X_{t} X_{t}^{\prime}\right)^{-1}, \\
\hat{V}_{2} & \equiv\left(T^{-1} \sum_{t=[T \lambda]+1}^{T} X_{t} X_{t}^{\prime}\right)^{-1}\left(T^{-4 / 3} \hat{h}_{2}(0)\right)\left(T^{-1} \sum_{t=[T \lambda]+1}^{T} X_{t} X_{t}^{\prime}\right)^{-1},
\end{aligned}
$$

with $\hat{h}_{1}(0)$ the HAC robust variance estimator using

$$
\hat{v}_{t}^{1}=\left\{\begin{array}{cc}
X_{t} e_{t}+X_{t} X_{t}^{\prime} \beta_{t}-X_{t} X_{t}^{\prime} \hat{\beta}_{1}, & \text { for } t \leq[T \lambda], \\
0, & \text { otherwise }
\end{array}\right.
$$

and $\hat{h}_{2}(0)$ using

$$
\hat{v}_{t}^{2}=\left\{\begin{array}{lr}
0, & \text { for } t \leq[T \lambda] \\
X_{t} e_{t}+X_{t} X_{t}^{\prime} \beta_{t}-X_{t} X_{t}^{\prime} \hat{\beta}_{2}, & \text { otherwise. }
\end{array}\right.
$$


As in the proof for $q \hat{L} L$, we can show that $\hat{h}_{1}(0)$ and $\hat{h}_{2}(0)$ are both $O_{p}\left(\|\Omega\|^{2} T^{2}\right)$ if $\left\{M-M_{11}\right\}_{i i}=0$ for some $i$. In this case,

$$
\begin{aligned}
S W \Rightarrow & \left(\frac{1}{\lambda^{*}} \Omega \int_{0}^{\lambda^{*}} W-\frac{1}{1-\lambda^{*}} \Omega \int_{\lambda^{*}}^{1} W\right)^{\prime} Q^{\prime} \\
& \times\left[\bar{h}_{1}(0)+\bar{h}_{2}(0)\right]^{-1} Q\left(\frac{1}{\lambda^{*}} \Omega \int_{0}^{\lambda^{*}} W-\frac{1}{1-\lambda^{*}} \Omega \int_{\lambda *}^{1} W\right)=O_{p}(1),
\end{aligned}
$$

where $\lambda^{*}$ is the value of $\lambda$ that maximizes the limit of the Wald test over the all permissible $\lambda$. If $\left\{M_{11}-M\right\}_{i i} \neq 0$ for all $i, \hat{h}_{1}(0)$ and $\hat{h}_{2}(0)$ are both $O_{p}\left(\|\Omega\|^{2} T^{4 / 3}\right)$ and $T^{-2 / 3} S W=O_{p}(1)$.

Proof of Theorem 3 (random walk parameters, lagged dependent variable): We now consider the dynamic regression. Under the null hypothesis, the OLS residuals are

$$
\hat{e}_{t}=e_{t}-\hat{\alpha} e_{t-1}+X_{t}^{\prime} \beta_{t}-\hat{\alpha} X_{t-1}^{\prime} \beta_{t-1}-X_{t}^{\prime} \hat{\beta}
$$

and

$$
\hat{v}_{t}=X_{t} e_{t}-\hat{\alpha} X_{t} e_{t-1}+X_{t} X_{t}^{\prime} \beta_{t}-\hat{\alpha} X_{t} X_{t-1}^{\prime} \beta_{t-1}-X_{t} X_{t}^{\prime} \hat{\beta},
$$

where the OLS estimates $\hat{\alpha}$ and $\hat{\beta}$ have the following limits:

$$
\begin{aligned}
\hat{\alpha} & \Rightarrow\left\{\int_{0}^{1} W^{\prime} \Omega^{\prime} Q \Omega W-\int_{0}^{1} W^{\prime} \Omega^{\prime} Q_{1}^{\prime} Q^{-1} Q_{1} \Omega \int_{0}^{1} W\right\}^{-1} \\
& \times\left\{\int_{0}^{1} W^{\prime} \Omega^{\prime} Q_{1} \Omega W-\int_{0}^{1} W^{\prime} \Omega^{\prime} Q_{1} \Omega \int_{0}^{1} W\right\} \equiv \bar{\alpha}
\end{aligned}
$$

and $T^{-1 / 2} \hat{\beta} \Rightarrow Q^{-1}\left[Q-\bar{\alpha} Q_{1}\right] \Omega \int_{0}^{1} W \equiv \bar{\beta}$. Consider first the $q \hat{L} L$ test. We take the limits of the components of (A.6). We have, $T^{-2} A_{3, T} \rightarrow 0_{k \times k}$,

$$
\begin{aligned}
& T^{-1} \hat{V}_{X}=T^{-2} \sum_{t=1}^{T}\left\{X_{t} X_{t}^{\prime} \beta_{t} \beta_{t}^{\prime} X_{t} X_{t}^{\prime}-\hat{\alpha} X_{t} X_{t}^{\prime} \beta_{t} \beta_{t-1}^{\prime} X_{t-1} X_{t}^{\prime}-X_{t} X_{t}^{\prime} \beta_{t} \hat{\beta}^{\prime} X_{t} X_{t}^{\prime}\right. \\
& -\hat{\alpha} X_{t} X_{t-1}^{\prime} \beta_{t-1} \beta_{t}^{\prime} X_{t} X_{t}^{\prime}+\hat{\alpha}^{2} X_{t} X_{t-1}^{\prime} \beta_{t-1} \beta_{t-1}^{\prime} X_{t-1} X_{t}^{\prime}+\hat{\alpha} X_{t} X_{t-1}^{\prime} \beta_{t-1} \hat{\beta}^{\prime} X_{t} X_{t}^{\prime} \\
& \left.-X_{t} X_{t}^{\prime} \hat{\beta} \beta_{t}^{\prime} X_{t} X_{t}^{\prime}+\hat{\alpha} X_{t} X_{t}^{\prime} \hat{\beta} \beta_{t-1}^{\prime} X_{t-1} X_{t}^{\prime}+X_{t} X_{t}^{\prime} \hat{\beta} \hat{\beta}^{\prime} X_{t} X_{t}^{\prime}\right\}+o_{p}(1) \\
\Rightarrow & M \Omega \int_{0}^{1} W W^{\prime} \Omega^{\prime} M^{\prime}-\bar{\alpha} M_{1} \Omega \int_{0}^{1} W W^{\prime} \Omega^{\prime} M_{1}^{\prime}-M \Omega W \bar{\beta}^{\prime} M^{\prime}-\bar{\alpha} M_{1} \Omega \int_{0}^{1} W W^{\prime} \Omega^{\prime} M_{1}^{\prime} \\
& +\bar{\alpha}^{2} M_{11} \Omega \int_{0}^{1} W W^{\prime} \Omega^{\prime} M_{11}^{\prime}+\bar{\alpha} M_{1} \Omega \int_{0}^{1} W \bar{\beta}^{\prime} M_{1}^{\prime}-M \bar{\beta} \int_{0}^{1} W^{\prime} \Omega^{\prime} M^{\prime} \\
& +\bar{\alpha} M_{1} \bar{\beta} \int_{0}^{1} W^{\prime} \Omega^{\prime} M_{1}^{\prime}+M \bar{\beta} \bar{\beta}^{\prime} M^{\prime} \\
= & M \Omega\left[\int_{0}^{1} W W^{\prime}-\int_{0}^{1} W \int_{0}^{1} W^{\prime}\right] \Omega^{\prime} M^{\prime}+\bar{\alpha}^{2} M Q^{-1} Q_{1} \Omega \int_{0}^{1} W \int_{0}^{1} W^{\prime} \Omega^{\prime} Q_{1}^{\prime} Q^{\prime-1} M^{\prime} \\
& +\bar{\alpha}^{2} M_{11} \Omega \int_{0}^{1} W W^{\prime} \Omega^{\prime} M_{11}^{\prime}-2 \bar{\alpha} M_{1} \Omega\left[\int_{0}^{1} W W^{\prime}-\int_{0}^{1} W \int_{0}^{1} W^{\prime}\right] \Omega^{\prime} M_{1}^{\prime} \\
& -\bar{\alpha}^{2} M_{1} Q^{-1} Q_{1} \Omega \int_{0}^{1} W \int_{0}^{1} W^{\prime} \Omega^{\prime} M_{1}^{\prime}-\bar{\alpha}^{2} M_{1} \Omega \int_{0}^{1} W \int_{0}^{1} W^{\prime} \Omega^{\prime} Q_{1}^{\prime} Q^{\prime-1} M_{1}^{\prime} \\
\equiv & \bar{V}_{X}=O_{p}\left(\|\Omega\|^{2}\right),
\end{aligned}
$$




$$
\begin{aligned}
& T^{-2} A_{1, T} \\
& =T^{-4} \sum_{t=1}^{T}\left\{\left[\sum_{s=1}^{t-1} \bar{r}^{s-1} X_{t-s} X_{t-s}^{\prime} \beta_{t-s}\right]\left(\left[\sum_{s=1}^{t-1} \bar{r}^{s-1} X_{t-s} X_{t-s}^{\prime} \beta_{t-s}\right]^{\prime}-\hat{\alpha}\left[\sum_{s=1}^{t-1} \bar{r}^{s-1} X_{t-s} X_{t-s-1}^{\prime} \beta_{t-s-1}\right]^{\prime}\right)\right. \\
& -\left[\sum_{s=1}^{t-1} \bar{r}^{s-1} X_{t-s} X_{t-s}^{\prime} \beta_{t-s}\right] \hat{\beta}^{\prime}\left[\sum_{s=1}^{t-1} \bar{r}^{s-1} X_{t-s} X_{t-s}^{\prime}\right]^{\prime} \\
& -\hat{\alpha}\left[\sum_{s=1}^{t-1} \bar{r}^{s-1} X_{t-s} X_{t-s-1}^{\prime} \beta_{t-s-1}\right]\left(\left[\sum_{s=1}^{t-1} \bar{r}^{s-1} X_{t-s} X_{t-s}^{\prime} \beta_{t-s}\right]^{\prime}-\hat{\alpha}\left[\sum_{s=1}^{t-1} \bar{r}^{s-1} X_{t-s} X_{t-s-1}^{\prime} \beta_{t-s-1}\right]^{\prime}\right) \\
& +\hat{\alpha}\left[\sum_{s=1}^{t-1} \bar{r}^{s-1} X_{t-s} X_{t-s-1}^{\prime} \beta_{t-s-1}\right] \hat{\beta}^{\prime}\left[\sum_{s=1}^{t-1} \bar{r}^{s-1} X_{t-s} X_{t-s}^{\prime}\right]^{\prime} \\
& -\left[\sum_{s=1}^{t-1} \bar{r}^{s-1} X_{t-s} X_{t-s}^{\prime}\right] \hat{\beta}\left(\left[\sum_{s=1}^{t-1} \bar{r}^{s-1} X_{t-s} X_{t-s}^{\prime} \beta_{t-s}\right]^{\prime}-\hat{\alpha} \hat{\beta}\left[\sum_{s=1}^{t-1} \bar{r}^{s-1} X_{t-s} X_{t-s-1}^{\prime} \beta_{t-s-1}\right]^{\prime}\right) \\
& \left.+\left[\sum_{s=1}^{t-1} \bar{r}^{s-1} X_{t-s} X_{t-s}^{\prime}\right] \hat{\beta} \hat{\beta}^{\prime}\left[\sum_{s=1}^{t-1} \bar{r}^{s-1} X_{t-s} X_{t-s}^{\prime}\right]^{\prime}\right\}+o_{p}(1) \\
& \Rightarrow \frac{1}{c^{2}}\left[Q-\bar{\alpha} Q_{1}\right] \Omega \int_{0}^{1}\left(W-W_{c}\right)\left(W-W_{c}\right)^{\prime} \Omega^{\prime}\left[Q-\bar{\alpha} Q_{1}\right]^{\prime} \\
& +\left[Q-\bar{\alpha} Q_{1}\right] \Omega \int_{0}^{1}\left(\int_{0}^{r} e^{-c \varsigma} d \varsigma\right)^{2} d r \int_{0}^{1} W \int_{0}^{1} W^{\prime} \Omega^{\prime}\left[Q-\bar{\alpha} Q_{1}\right]^{\prime} \\
& -\frac{1}{c}\left[Q-\bar{\alpha} Q_{1}\right] \Omega \int_{0}^{1}\left(\int_{0}^{r} e^{-c \varsigma} d \varsigma\right)\left(W-W_{c}\right) d r \int_{0}^{1} W^{\prime} \Omega^{\prime}\left[Q-\bar{\alpha} Q_{1}\right]^{\prime} \\
& -\frac{1}{c}\left[Q-\bar{\alpha} Q_{1}\right] \Omega \int_{0}^{1} W \int_{0}^{1}\left(\int_{0}^{r} e^{-c \varsigma} d \varsigma\right)\left(W-W_{c}\right) d r^{\prime} \Omega^{\prime}\left[Q-\bar{\alpha} Q_{1}\right]^{\prime}=O_{p}\left(\|\Omega\|^{2}\right), \\
& T^{-2} A_{2, T} \\
& =T^{-3} \sum_{t=1}^{T} X_{t} X_{t}^{\prime}\left\{\beta_{t}\left[\sum_{s=1}^{t-1} \bar{r}^{s-1} X_{t-s} X_{t-s}^{\prime} \beta_{t-s}\right]^{\prime}-\hat{\alpha} \beta_{t}\left[\sum_{s=1}^{t-1} \bar{r}^{s-1} X_{t-s} X_{t-s-1}^{\prime} \beta_{t-s-1}\right]^{\prime}\right. \\
& -\beta_{t}\left[\sum_{s=1}^{t-1} \bar{r}^{s-1} X_{t-s} X_{t-s}^{\prime} \hat{\beta}\right]^{\prime}-\hat{\beta}\left[\sum_{s=1}^{t-1} \bar{r}^{s-1} X_{t-s} X_{t-s}^{\prime} \beta_{t-s}\right]^{\prime}+\hat{\alpha} \hat{\beta}\left[\sum_{s=1}^{t-1} \bar{r}^{s-1} X_{t-s} X_{t-s-1}^{\prime} \beta_{t-s-1}\right]^{\prime} \\
& \left.+\hat{\beta}\left[\sum_{s=1}^{t-1} \bar{r}^{s-1} X_{t-s} X_{t-s}^{\prime} \hat{\beta}\right]^{\prime}\right\}-\hat{\alpha} T^{-3} \sum_{t=1}^{T} X_{t} X_{t-1}^{\prime}\left\{\beta_{t-1}\left[\sum_{s=1}^{t-1} \bar{r}^{s-1} X_{t-s} X_{t-s}^{\prime} \beta_{t-s}\right]^{\prime}\right. \\
& \left.-\hat{\alpha} \beta_{t-1}\left[\sum_{s=1}^{t-1} \bar{r}^{s-1} X_{t-s} X_{t-s-1}^{\prime} \beta_{t-s-1}\right]^{\prime}-\beta_{t-1}\left[\sum_{s=1}^{t-1} \bar{r}^{s-1} X_{t-s} X_{t-s}^{\prime} \hat{\beta}\right]^{\prime}\right\}+o_{p}(1) \\
& \Rightarrow \frac{1}{c}\left[Q-\bar{\alpha} Q_{1}\right] \Omega \int_{0}^{1}\left(W W^{\prime}-W W_{c}^{\prime}\right) \Omega^{\prime}\left[Q-\bar{\alpha} Q_{1}\right]^{\prime} \\
& -\left[Q-\bar{\alpha} Q_{1}\right] \Omega \int_{0}^{1}\left(\int_{0}^{r} e^{-c \varsigma} d \varsigma\right) W d r \int_{0}^{1} W^{\prime} \Omega^{\prime}\left[Q-\bar{\alpha} Q_{1}\right]^{\prime} \\
& -\frac{1}{c}\left[Q-\bar{\alpha} Q_{1}\right] \Omega \int_{0}^{1} W \int_{0}^{1}\left(W-W_{c}\right)^{\prime} \Omega^{\prime}\left[Q-\bar{\alpha} Q_{1}\right]^{\prime} \\
& +\left[Q-\bar{\alpha} Q_{1}\right] \Omega \int_{0}^{1} \int_{0}^{r} e^{-c \varsigma} d \varsigma d r \int_{0}^{1} W \int_{0}^{1} W^{\prime} \Omega^{\prime}\left[Q-\bar{\alpha} Q_{1}\right]^{\prime}=O_{p}\left(\|\Omega\|^{2}\right),
\end{aligned}
$$




$$
\begin{aligned}
T^{-1} A_{4, T} & =T^{-3 / 2} \sum_{t=1}^{T} \bar{r}^{t} X_{t} X_{t}^{\prime} \beta_{t}-\hat{\alpha} T^{-3 / 2} \sum_{t=1}^{T} \bar{r}^{t} X_{t} X_{t-1}^{\prime} \beta_{t-1}-T^{-3 / 2} \sum_{t=1}^{T} \bar{r}^{t} X_{t} X_{t}^{\prime} \hat{\beta}+o_{p}(1) \\
& \Rightarrow\left[Q-\bar{\alpha} Q_{1}\right] \Omega \int_{0}^{1} e^{-c r} W-\left[Q-\bar{\alpha} Q_{1}\right] \Omega \int_{0}^{1} e^{-c r} \int_{0}^{1} W=O_{p}(\|\Omega\|) \\
T^{-1} A_{5, T} & =T^{-5 / 2} \sum_{t=1}^{T} \bar{r}^{t} \sum_{s=1}^{t-1} \bar{r}^{s-1}\left\{X_{t-s} X_{t-s}^{\prime} \beta_{t-s}-\hat{\alpha} X_{t-s} X_{t-s-1}^{\prime} \beta_{t-s-1}-X_{t-s} X_{t-s}^{\prime} \hat{\beta}\right\}+o_{p}(1) \\
& \Rightarrow \frac{1}{c}\left[Q-\bar{\alpha} Q_{1}\right] \Omega \int_{0}^{1} e^{-c r}\left(W-W_{c}\right)-\left[Q-\bar{\alpha} Q_{1}\right] \Omega \int_{0}^{1} e^{-c r} \int_{0}^{r} e^{-c s} d s d r \int_{0}^{1} W=O_{p}(\|\Omega\|) .
\end{aligned}
$$

Collecting terms according to (A.6) yields the stated result. We now consider the sup-Wald test. The regression is

$$
y_{t}=\alpha_{u} y_{t-1}+X_{1}(\lambda)_{t}^{\prime} \beta_{1}+X_{2}(\lambda)_{t}^{\prime} \beta_{2}+e_{t}
$$

and the OLS estimates are

$$
\left[\begin{array}{c}
\hat{\alpha}_{u} \\
T^{-1 / 2}\left[\hat{\beta}_{1}, \hat{\beta}_{2}\right]^{\prime}
\end{array}\right]=\left[\begin{array}{cc}
T^{-2} \sum_{t=2}^{T} y_{t-1}^{2} & T^{-3 / 2} \sum_{t=2}^{T} y_{t-1} \bar{X}(\lambda)_{t}^{\prime} \\
T^{-3 / 2} \sum_{t=2}^{T} \bar{X}(\lambda)_{t} y_{t-1} & T^{-1} \sum_{t=2}^{T} \bar{X}(\lambda)_{t} \bar{X}(\lambda)_{t}^{\prime}
\end{array}\right]^{-1}\left[\begin{array}{c}
T^{-2} \sum_{t=2}^{T} y_{t-1} y_{t} \\
T^{-3 / 2} \sum_{t=2}^{T} \bar{X}(\lambda)_{t} y_{t}
\end{array}\right]
$$

where $\bar{X}(\lambda)_{t}=\left[X_{1}(\lambda)_{t}, X_{2}(\lambda)_{t}\right]$. After some algebra, we obtain

$$
\begin{aligned}
\hat{\alpha}_{u} \Rightarrow & \left\{\int_{0}^{1} W^{\prime} \Omega^{\prime} Q \Omega W-\frac{1}{\lambda} \int_{0}^{\lambda} W^{\prime} \Omega^{\prime} Q_{1}^{\prime} Q^{-1} Q_{1} \Omega \int_{0}^{\lambda} W-\frac{1}{1-\lambda} \int_{\lambda}^{1} W^{\prime} \Omega^{\prime} Q_{1}^{\prime} Q^{-1} Q_{1} \Omega \int_{\lambda}^{1} W\right\}^{-1} \\
& \times\left\{\int_{0}^{1} W^{\prime} \Omega^{\prime} Q_{1} \Omega W-\frac{1}{\lambda} \int_{0}^{\lambda} W^{\prime} \Omega^{\prime} Q_{1} \Omega \int_{0}^{\lambda} W-\frac{1}{1-\lambda} \int_{\lambda}^{1} W^{\prime} \Omega^{\prime} Q_{1} \Omega \int_{\lambda}^{1} W\right\} \equiv \bar{\alpha}_{u}, \quad(\mathrm{~A} .13)
\end{aligned}
$$

and

$$
\begin{aligned}
T^{-1 / 2} \hat{\beta}_{1} & \Rightarrow \frac{1}{\lambda} \Omega Q^{-1}\left[Q-\bar{\alpha}_{u} Q_{1}\right] \int_{0}^{\lambda} W \\
T^{-1 / 2} \hat{\beta}_{2} & \Rightarrow \frac{1}{1-\lambda} \Omega Q^{-1}\left[Q-\bar{\alpha}_{u} Q_{1}\right] \int_{\lambda}^{1} W
\end{aligned}
$$

Using these results, the limit of $S S R(\lambda)_{T}$ is given by:

$$
\begin{aligned}
T^{-2} S S R(\lambda)_{T}= & T^{-2} \sum_{t=1}^{T}\left[X_{t}^{\prime} \beta_{t}-\hat{\alpha} X_{t-1}^{\prime} \beta_{t-1}-X_{1}(\lambda)_{t}^{\prime} \hat{\beta}_{1}-X_{2}(\lambda)_{t}^{\prime} \hat{\beta}_{2}\right]^{2}+o_{p}(1) \\
\Rightarrow & \left(1+\bar{\alpha}_{u}^{2}\right) \int_{0}^{1} W^{\prime} \Omega^{\prime} Q \Omega W-2 \bar{\alpha}_{u} \int_{0}^{1} W^{\prime} \Omega^{\prime} Q_{1} \Omega W \\
& -\frac{1}{\lambda} \int_{0}^{\lambda} W^{\prime} \Omega^{\prime}\left[Q-\bar{\alpha}_{u} Q_{1}\right]^{\prime} Q^{-1}\left[Q-\bar{\alpha}_{u} Q_{1}\right] \Omega \int_{0}^{\lambda} W \\
& -\frac{1}{1-\lambda} \int_{\lambda}^{1} W^{\prime} \Omega^{\prime}\left[Q-\bar{\alpha}_{u} Q_{1}\right]^{\prime} Q^{-1}\left[Q-\bar{\alpha}_{u} Q_{1}\right] \Omega \int_{\lambda}^{1} W \equiv S S R(\lambda)^{*}=O_{p}\left(\|\Omega\|^{2}\right),
\end{aligned}
$$


where $\bar{\alpha}_{u}$ is defined by (A.13). The limit of the restricted sum of squared residuals is

$$
\begin{aligned}
& T^{-2} S S R_{T}^{r} \\
= & T^{-2} \sum_{t=1}^{T}\left[X_{t}^{\prime} \beta_{t}-\hat{\alpha} X_{t-1}^{\prime} \beta_{t-1}-X_{t} \hat{\beta}\right]^{2}+o_{p}(1) \\
\Rightarrow & \left(1+\bar{\alpha}^{2}\right) \int_{0}^{1} W^{\prime} \Omega^{\prime} Q \Omega W-2 \bar{\alpha} \int_{0}^{1} W^{\prime} \Omega^{\prime} Q_{1} \Omega W-\int_{0}^{1} W^{\prime} \Omega^{\prime}\left[Q-\bar{\alpha} Q_{1}\right]^{\prime} Q^{-1}\left[Q-\bar{\alpha} Q_{1}\right] \Omega \int_{0}^{1} W \\
\equiv & S S R^{r *}=O_{p}\left(\|\Omega\|^{2}\right),
\end{aligned}
$$

where $\bar{\alpha}$ is defined by (A.12). By the continuous mapping theorem, the limit of the sup-Wald test is given by

$$
T^{-1} S W=\left(\frac{T^{-2} S S R_{T}^{r}-T^{-2} S S R_{T}\left(\bar{\lambda}_{T}\right)}{T^{-2} S S R_{T}\left(\bar{\lambda}_{T}\right)}\right) \Rightarrow\left(\frac{S S R^{r *}-S S R^{*}(\bar{\lambda})}{S S R^{*}(\bar{\lambda})}\right)=O_{p}(1),
$$

with $\bar{\lambda}_{T}$ the value that minimizes $S S R_{T}(\lambda)$ and $\bar{\lambda}$ minimizes $S S R^{*}(\lambda)$. We next consider the special case of a mean shift model when $x_{t}=1$ for all $t$ with the dynamic regression. For the restricted model, the regression equation is

$$
y_{t}=\alpha y_{t-1}+\beta+e_{t}
$$

and the OLS estimates are such that

$$
\begin{gathered}
T(\hat{\alpha}-1)=\frac{T^{-1} \sum_{t=2}^{T} y_{t-1}\left(\varepsilon_{t}+e_{t}-e_{t-1}\right)-\left[T^{-3 / 2} \sum_{t=2}^{T} y_{t-1}\right]\left[T^{-1 / 2} \sum_{t=2}^{T}\left(\varepsilon_{t}+e_{t}-e_{t-1}\right)\right]}{T^{-2} \sum_{t=2}^{T} y_{t-1}^{2}-\left[T^{-3 / 2} \sum_{t=2}^{T} y_{t-1}\right]^{2}} \\
\Rightarrow \frac{\frac{1}{2}\left[W(1)^{2}-1\right]-\sigma_{e}^{2} / \Omega^{2}-W(1) \int_{0}^{1} W}{\int_{0}^{1} W^{2}-\left(\int_{0}^{1} W\right)^{2}} \equiv \bar{\alpha}=O_{p}(1), \\
T^{1 / 2} \hat{\beta} \Rightarrow-\bar{\alpha} \Omega \int_{0}^{1} W+\Omega W(1) \equiv \bar{\beta} .
\end{gathered}
$$

For the unrestricted model, the regression equation is

$$
y_{t}=\alpha_{u} y_{t-1}+\beta_{1} I_{t}^{1}+\beta_{2} I_{t}^{2}+e_{t},
$$

where $I_{t}^{1}=1$ if $t<[T \lambda]$ and 0 otherwise and $I_{t}^{2}=0$ if $t<[T \lambda]$ and 1 otherwise. Let $I_{t}=\left[I_{t}^{1}, I_{t}^{2}\right]$. The OLS estimates are such that

$$
\begin{aligned}
& T\left(\hat{\alpha}_{u}-1\right) \\
= & \frac{T^{-1} \sum_{t=2}^{T} y_{t-1}\left(\varepsilon_{t}+e_{t}-e_{t-1}\right)-\left[T^{-3 / 2} \sum_{t=2}^{T} y_{t-1} I_{t}^{\prime}\right]\left[T^{-1} \sum_{t=2}^{T} I_{t} I_{t}^{\prime}\right]\left[T^{-1 / 2} \sum_{t=2}^{T} I_{t}\left(\varepsilon_{t}+e_{t}-e_{t-1}\right)\right]}{T^{-2} \sum_{t=2}^{T} y_{t-1}^{2}-\left[T^{-3 / 2} \sum_{t=2}^{T} y_{t-1}\right]^{2}} \\
\Rightarrow & \frac{\frac{1}{2}\left[W(1)^{2}-1\right]-\sigma_{e}^{2} / \Omega^{2}-\frac{1}{\lambda} \int_{0}^{\lambda} W W(\lambda)-\frac{1}{1-\lambda} \int_{\lambda}^{1} W W(1-\lambda)}{\int_{0}^{1} W^{2}-\frac{1}{\lambda}\left(\int_{0}^{\lambda} W\right)^{2}-\frac{1}{1-\lambda}\left(\int_{\lambda}^{1} W\right)^{2}} \equiv \bar{\alpha}_{u}=O_{p}(1),
\end{aligned}
$$




$$
\begin{aligned}
& T^{1 / 2} \hat{\beta}_{1} \quad \Rightarrow \quad-\bar{\alpha}_{u} \frac{1}{\lambda} \Omega \int_{0}^{\lambda} W+\frac{1}{\lambda} \Omega W(\lambda) \equiv \bar{\beta}_{1}, \\
& T^{1 / 2} \hat{\beta}_{2} \Rightarrow-\bar{\alpha}_{u} \frac{1}{1-\lambda} \Omega \int_{\lambda}^{1} W+\frac{1}{1-\lambda} \Omega W(1-\lambda) \equiv \bar{\beta}_{2} .
\end{aligned}
$$

Therefore, we have $\hat{\alpha}, \hat{\alpha}_{u} \stackrel{p}{\rightarrow} 1$ and $\hat{\beta}, \hat{\beta}_{1}, \hat{\beta}_{2} \stackrel{p}{\rightarrow} 0$. Also, $T\left(\hat{\alpha}-\hat{\alpha}_{u}\right) \Rightarrow \bar{\alpha}-\bar{\alpha}_{u}$ and $T\left(\hat{\alpha}^{2}-\hat{\alpha}_{u}^{2}\right) \Rightarrow$ $2\left(\bar{\alpha}-\bar{\alpha}_{u}\right)$. Consider first the $q \hat{L} L$ test. Since $x_{t}=1$ for all $t, \hat{v}_{t}=\hat{e}_{t}$ and

$$
\hat{v}_{t}=e_{t}-\hat{\alpha} e_{t-1}+\beta_{t}-\hat{\alpha} \beta_{t-1}-\hat{\beta} \text {. }
$$

The components in (A.6) are such that (since $e_{t}$ and $\varepsilon_{t}$ are uncorrelated)

$$
\begin{aligned}
\hat{V}_{X} & =T^{-1} \sum_{t=2}^{T} \hat{v}_{t}^{2} \stackrel{p}{\rightarrow} \Omega^{2}+2 \sigma_{e}^{2} \\
A_{1, T} & =T^{-2} \sum_{t=2}^{T}\left[\sum_{s=1}^{t-1} \bar{r}^{s-1}\left(\varepsilon_{t-s}+e_{t-s}-e_{t-s-1}\right)\right]^{2}+o_{p}(1) \Rightarrow \Omega^{2} \int_{0}^{1} W_{c}^{2} \\
A_{2, T} & =T^{-1} \sum_{t=2}^{T}\left(\varepsilon_{t}+e_{t}-e_{t-1}\right)\left[\sum_{s=1}^{t-1} \bar{r}^{s-1}\left(\varepsilon_{t-s}+e_{t-s}-e_{t-s-1}\right)\right]+o_{p}(1) \Rightarrow 0 \\
A_{3, T} & =T^{-1} \sum_{t=2}^{T} \hat{v}_{t}^{2} \stackrel{p}{\rightarrow} \Omega^{2}+2 \sigma_{e}^{2} \\
A_{4, T} & =T^{-1 / 2} \sum_{t=2}^{T} \bar{r}^{t}\left(\varepsilon_{t}+e_{t}-e_{t-1}\right)+o_{p}(1) \Rightarrow \Omega W_{c}(1), \\
A_{5, T} & =T^{-3 / 2} \sum_{t=2}^{T} \bar{r}^{t} \sum_{s=1}^{t-1}\left(\varepsilon_{t-s}+e_{t-s}-e_{t-s-1}\right)+o_{p}(1) \Rightarrow \Omega \int_{0}^{1} e^{-c r} W_{c} .
\end{aligned}
$$

Therefore,

$$
q \hat{L} L \Rightarrow \frac{c^{2} \Omega^{2} \int_{0}^{1} W_{c}^{2}-c \Omega^{2}-2 c \sigma_{e}^{2}-\left(2 c \Omega^{2} / 1-e^{-2 c}\right)\left[W_{c}(1)-c \int_{0}^{1} e^{-c r} W_{c}\right]^{2}}{\Omega^{2}+2 \sigma_{e}^{2}} .
$$

We next consider the sup-Wald test, $\operatorname{Wald}(\lambda)_{T}=\left(S S R_{T}^{r}-S S R_{T}(\lambda)\right) / T^{-1} S S R_{T}(\lambda)$. Using the expression for the restricted and the unrestricted residuals yields

$$
\begin{aligned}
& S S R_{T}^{r}-S S R_{T}(\lambda) \\
= & \sum_{t=2}^{T}\left[y_{t}-\hat{\alpha} y_{t-1}-\hat{\beta}\right]^{2}-\sum_{t=2}^{T}\left[y_{t}-\hat{\alpha}_{u} y_{t-1}-\hat{\beta}_{1} I_{t \leq[T \lambda]}-\hat{\beta}_{2} I_{t>[T \lambda]}\right]^{2}+o_{p}(1) \\
= & \left(\hat{\alpha}^{2}-\hat{\alpha}_{u}^{2}\right) \sum_{t=2}^{T} y_{t-1}^{2}+T \hat{\beta}^{2}-[T \lambda] \hat{\beta}_{1}^{2}-(T-[T \lambda]) \hat{\beta}_{2}^{2} \\
& -2\left(\hat{\alpha}-\hat{\alpha}_{u}\right) \sum_{t=2}^{T} y_{t} y_{t-1}-2(1-\hat{\alpha}) \hat{\beta} \sum_{t=2}^{T} y_{t-1}+2\left(1-\hat{\alpha}_{u}\right)\left[\hat{\beta}_{1} \sum_{t=2}^{[T \lambda]} y_{t-1}+\hat{\beta}_{2} \sum_{t=[T \lambda]+1}^{T} y_{t-1}\right]+o_{p}(1) \\
\Rightarrow & -2\left(\bar{\alpha}-\bar{\alpha}_{u}\right)\left[(1 / 2) \Omega^{2}(W(1)-1)-\sigma_{e}^{2}\right]+\Omega^{2} W(1)^{2}-\Omega^{2} W(\lambda)^{2}-\Omega^{2} W(1-\lambda)^{2} \\
& -\bar{\alpha}^{2} \Omega^{2}\left(\int_{0}^{1} W\right)^{2}+\bar{\alpha}_{u}^{2} \frac{1}{\lambda} \Omega^{2}\left(\int_{0}^{\lambda} W\right)^{2}+\bar{\alpha}_{u}^{2} \frac{1}{1-\lambda} \Omega^{2}\left(\int_{\lambda}^{1} W\right)^{2} \\
\equiv & N=O_{p}\left(\left\|\Omega^{2}\right\|\right)
\end{aligned}
$$


and

$$
\begin{aligned}
T^{-1} S S R_{T}(\lambda) & =\sum_{t=2}^{T}\left[y_{t}-\hat{\alpha}_{u} y_{t-1}-\hat{\beta}_{1} I_{t \leq[T \lambda]}-\hat{\beta}_{2} I_{t>[T \lambda]}\right]^{2} \\
& =T^{-1} y_{T}^{2}-2 \hat{\alpha}_{u} T^{-1} \sum_{t=2}^{T} y_{t-1}\left(\varepsilon_{t}+e_{t}-e_{t-1}\right) \\
& \Rightarrow \Omega^{2}+2 \sigma_{e}^{2} \equiv D=O_{p}\left(\left\|\Omega^{2}\right\|\right) .
\end{aligned}
$$

Therefore by the continuous mapping theorem $S W \Rightarrow N / D$.

Proof of Theorem 4 (infrequent break parameter; i.i.d. errors assumed): For the $q \hat{L} L$ test, the OLS estimate of $\beta$ is given by

$$
\hat{\beta}=\left(T^{-1} \sum_{t=1}^{T} X_{t} X_{t}^{\prime}\right)^{-1}\left(T^{-1} \sum_{t=1}^{T} X_{t} X_{t}^{\prime} \beta_{t}+T^{-1} \sum_{t=1}^{T} X_{t} e_{t}\right) \Rightarrow \int_{0}^{1} J(s) d s .
$$

Using the expression (A.8) for $\hat{v}_{t}$, the limit of the variance is

$$
\hat{V}_{X}=T^{-1} \sum_{t=1}^{T} \hat{v}_{t} \hat{v}_{t}^{\prime} \Rightarrow \sigma_{e}^{2} Q+M \Omega\left[\int_{0}^{1} J J^{\prime}-\int_{0}^{1} J \int_{0}^{1} J^{\prime}\right] \Omega^{\prime} M^{\prime} \equiv \bar{V}_{X}=O_{p}\left(\|\Omega\|^{2}\right)
$$

The components of (A.6) are such that (again, $T^{-1} A_{3, T} \rightarrow 0_{k \times k}$ )

$$
\begin{gathered}
T^{-1} A_{1, T} \\
=T^{-3} \sum_{t=1}^{T}\left[\sum_{s=1}^{t-1} \bar{r}^{s-1} \hat{v}_{t-s}\right]\left[\sum_{s=1}^{t-1} \bar{r}^{s-1} \hat{v}_{t-s}\right]^{\prime} \\
\Rightarrow \quad Q \int_{0}^{1}\left[\int_{0}^{r} e^{-c \varsigma} J(r-\varsigma) d \varsigma\right]\left[\int_{0}^{r} e^{-c \varsigma} J(r-\varsigma) d \varsigma\right]^{\prime} d r \Omega^{\prime} Q^{\prime} \\
-Q \Omega \int_{0}^{1} \int_{0}^{r} e^{-c \varsigma} d \varsigma \int_{0}^{r} e^{-c \varsigma} J(r-\varsigma) d \varsigma d r \int_{0}^{1} J^{\prime} \Omega^{\prime} Q^{\prime} \\
-Q \Omega \int_{0}^{1} J \int_{0}^{1} \int_{0}^{r} e^{-c \varsigma} d \varsigma \int_{0}^{r} e^{-c \varsigma} J(r-\varsigma)^{\prime} d \varsigma d r \Omega^{\prime} Q^{\prime}+Q \Omega \int_{0}^{1} \int_{0}^{r} e^{-c \varsigma} d \varsigma^{2} d r \int_{0}^{1} J \int_{0}^{1} J^{\prime} \Omega^{\prime} Q^{\prime} \\
\equiv \Omega \Psi_{1} \Omega^{\prime} Q^{\prime}=O_{p}\left(\|\Omega\|^{2}\right), \\
T^{-1} A_{2, T}=T^{-2} \sum_{t=1}^{T} \hat{v}_{t}\left[\sum_{s=1}^{t-1} \bar{r}^{s-1} \hat{v}_{t-s}\right]^{\prime} \\
\Rightarrow Q \Omega \int_{0}^{1} J(r) \int_{0}^{r} e^{-c \varsigma} J(r-\varsigma) d \varsigma d r \Omega^{\prime} Q^{\prime}-Q \Omega \int_{0}^{1} \int_{0}^{r} e^{-c \varsigma} d \varsigma J(r) d r \int_{0}^{1} J^{\prime} \Omega^{\prime} Q^{\prime} \\
\quad-Q \Omega \int_{0}^{1} J \int_{0}^{1} \int_{0}^{r} e^{-c \varsigma} J(r-\varsigma) d \varsigma d r^{\prime} \Omega^{\prime} Q^{\prime}+Q \Omega \int_{0}^{1} \int_{0}^{r} e^{-c \varsigma} d \varsigma d r \int_{0}^{1} J \int_{0}^{1} J^{\prime} \Omega^{\prime} Q^{\prime} \\
\equiv Q \Omega \Psi_{2} \Omega^{\prime} Q^{\prime}=O_{p}\left(\|\Omega\|^{2}\right), \\
T^{-1 / 2} A_{4, T}=T^{-1} \sum_{t=1}^{T} \bar{r}^{t} \hat{v}_{t} \Rightarrow Q \Omega \int_{0}^{1} e^{-c r} J-Q \Omega \int_{0}^{1} e^{-c r} \int_{0}^{1} J \equiv Q \Omega \Psi 4=O p(\|\Omega\|),
\end{gathered}
$$




$$
\begin{aligned}
T^{-1 / 2} A_{5, T} & =T^{-2} \sum_{t=1}^{T} \bar{r}^{t} \sum_{s=1}^{t-1} \bar{r}^{s-1} \hat{v}_{t-s} \\
& \Rightarrow Q \Omega \int_{0}^{1} e^{-c r} \int_{0}^{r} e^{-c \varsigma} J(r-\varsigma) d \varsigma d r-Q \Omega \int_{0}^{1} e^{-c r} \int_{0}^{r} e^{-c \varsigma} d \varsigma d r \int_{0}^{1} J \\
& \equiv Q \Omega \Psi_{5}=O_{p}(\|\Omega\|) .
\end{aligned}
$$

Solving the integrals and collecting terms according to (A.6) yields

$$
T^{-1} q \hat{L} L \Rightarrow \operatorname{tr}\left\{\bar{V}_{X}^{-1 / 2} Q \Omega \Psi \Omega^{\prime} Q^{\prime} \bar{V}^{\prime-1 / 2}\right\}
$$

where

$$
\Psi=c^{2} \Psi_{1}-c\left(\Psi_{2}+\Psi_{2}^{\prime}\right)-\frac{2 c}{1-e^{-2 c}}\left[\Psi_{4}-c \Psi_{5}\right]^{\prime}\left[\Psi_{4}-c \Psi_{5}\right] .
$$

We next consider the sup-Wald test. The unrestricted residuals are given by (A.9) and we have $\hat{\beta}_{1} \Rightarrow(1 / \lambda) \Omega \int_{0}^{\lambda} J$ and $\hat{\beta}_{2} \Rightarrow(1 /(1-\lambda)) \Omega \int_{\lambda}^{1} J$. Hence, after some algebra, we get

$$
\begin{aligned}
T^{-1} S S R(\lambda)_{T} & \Rightarrow \sigma_{e}^{2}+\int_{0}^{1} J^{\prime} \Omega^{\prime} Q \Omega J-\frac{1}{\lambda} \int_{0}^{\lambda} J^{\prime} \Omega^{\prime} Q \Omega \int_{0}^{\lambda} J-\frac{1}{1-\lambda} \int_{\lambda}^{1} J^{\prime} \Omega^{\prime} Q \Omega \int_{\lambda}^{1} J \\
& \equiv S S R^{*}(\lambda)=O_{p}\left(\|\Omega\|^{2}\right) .
\end{aligned}
$$

For the restricted regression,

$$
T^{-1} S S R_{T}^{r} \Rightarrow \sigma_{e}^{2}+\int_{0}^{1} J^{\prime} \Omega^{\prime} Q \Omega J-\int_{0}^{1} J^{\prime} \Omega^{\prime} Q \Omega \int_{0}^{1} J \equiv S S R^{r *}=O_{p}\left(\|\Omega\|^{2}\right) .
$$

Therefore by the continuous mapping theorem,

$$
T^{-1} S W=\left(\frac{T^{-1} S S R_{T}^{r}-T^{-1} S S R_{T}\left(\bar{\lambda}_{T}\right)}{T^{-1} S S R_{T}\left(\bar{\lambda}_{T}\right)}\right) \Rightarrow\left(\frac{S S R^{r *}-S S R^{*}(\bar{\lambda})}{S S R^{*}(\bar{\lambda})}\right),
$$

where $\bar{\lambda}_{T}$ is the value of $\lambda$ that minimizes $S S R_{T}(\lambda)$ and $\bar{\lambda}$ is the value that minimizes $S S R^{*}(\bar{\lambda})$.

Proof of Theorem 5 (infrequent break parameter; correction for serial correlation): For the $q \hat{L} L$ test, the limit of $\hat{\Gamma}_{j}$ is given by

$$
\begin{aligned}
\hat{\Gamma}_{j} & =T^{-1} \sum_{t=j+1}^{T} X_{t} X_{t}^{\prime} \beta_{t} \beta_{t-j}^{\prime} X_{t-j} X_{t-j}^{\prime}-T^{-1} \sum_{t=j+1}^{T} X_{t} X_{t}^{\prime} \beta_{t}\left(\sum_{s=1}^{T} \beta_{s}^{\prime}\right) X_{t-j} X_{t-j}^{\prime}+o_{p}(1) \\
& \Rightarrow M_{j j} \Omega\left[\int_{0}^{1} J J^{\prime}-\int_{0}^{1} J \int_{0}^{1} J^{\prime}\right] \Omega^{\prime} M_{j j}^{\prime} \equiv \bar{\Gamma}_{j}=O_{p}\left(\|\Omega\|^{2}\right)
\end{aligned}
$$

uniformly in $j=o(T)$. The estimated bandwidth involves

$$
\bar{\alpha} \equiv \sum_{i=1}^{k} \frac{4 \bar{\rho}_{i}^{2} \bar{\sigma}_{v i}^{4}}{\left(1-\bar{\rho}_{i}\right)^{6}\left(1+\bar{\rho}_{i}\right)^{2}} / \sum_{i=1}^{k} \frac{\bar{\sigma}_{v i}^{4}}{\left(1-\bar{\rho}_{i}\right)^{4}}
$$

where

$$
\hat{\rho}_{i} \Rightarrow \frac{\left\{M_{11}\right\}_{i i}^{2}\{\Omega\}_{i i}^{2}\left[\int_{0}^{1} J_{i}^{2}-\left(\int_{0}^{1} J_{i}\right)^{2}\right]}{\sigma_{e}^{2}\{Q\}_{i i}+\{M\}_{i i}^{2}\{\Omega\}_{i i}^{2}\left[\int_{0}^{1} J_{i}^{2}-\left(\int_{0}^{1} J_{i}\right)^{2}\right]} \equiv \bar{\rho}_{i}
$$


and $\bar{\sigma}_{v i}^{4}$ is bounded for the same reason as Theorem 2. In $\bar{\rho}_{i}$, the term $\sigma_{e}^{2}\{Q\}_{i i}$ is dominated as $\|\Omega\|$ increases, and if $\left\{M-M_{11}\right\}_{i i}=0, \hat{\rho}_{i}$ converges to 1 at rate $\|\Omega\|^{2}$. Therefore, the $i$ th component such that $\left\{M-M_{11}\right\}_{i i}=0$ dominates in both the numerator and denominator of $\alpha$ and yields $\alpha=O_{p}\left(\|\Omega\|^{4}\right)$ and $m=O_{p}\left(\|\Omega\|^{4 / 3} T^{1 / 3}\right)$, so that $\hat{h}(0)=O_{p}\left(\|\Omega\|^{10 / 3} T^{1 / 3}\right)$. Since $\hat{V}_{X}=O_{p}\left(\|\Omega\|^{2}\right)$, the result follows. For the sup-Wald test, we construct $\hat{h}_{l}(0)$ using the unrestricted residuals and we have,

$$
\begin{aligned}
\bar{\rho}_{1, i} & \equiv \frac{\left\{M_{11}\right\}_{i i}^{2}\{\Omega\}_{i i}^{2}\left[\left[\int_{0}^{\lambda^{*}} J_{i}^{2}-\left(\int_{0}^{\lambda^{*}} J_{i}\right)^{2}\right]\right.}{\sigma_{e}^{2}\{Q\}_{i i}+\{M\}_{i i}^{2}\{\Omega\}_{i i}^{2}\left[\left[\int_{0}^{\lambda^{*}} J_{i}^{2}-\left(\int_{0}^{\lambda^{*}} J_{i}\right)^{2}\right]\right.} \\
\bar{\rho}_{2, i} \equiv & \equiv \frac{\left\{M_{11}\right\}_{i i}^{2}\{\Omega\}_{i i}^{2}\left[\int_{\lambda^{*}}^{1} J_{i}^{2}-\left(\int_{\lambda^{*}}^{1} J_{i}\right)^{2}\right]}{\sigma_{e}^{2}\{Q\}_{i i}+\{M\}_{i i}^{2}\{\Omega\}_{i i}^{2}\left[\int_{\lambda^{*}}^{1} J_{i}^{2}-\left(\int_{\lambda^{*}}^{1} J_{i}\right)^{2}\right]} .
\end{aligned}
$$

Using similar arguments to the $q \hat{L} L$ case, $\hat{h}(0)=O_{p}\left(\|\Omega\|^{10 / 3} T^{1 / 3}\right)$. If $\left\{M-M_{11}\right\}_{i i} \neq 0$ for some $i$, then $\alpha=O_{p}(1)$ and $m=O_{p}\left(T^{1 / 3}\right)$. This yields $\hat{h}(0)=O_{p}\left(\|\Omega\|^{2} T^{1 / 3}\right)$ for both the $q \hat{L} L$ and sup-Wald tests. With these orders for $\hat{h}(0)$, the rest of the proof is the same as for the random walk case.

Proof of Theorem 6 (infrequent break parameter; lagged dependent variable): We now consider the dynamic regression. As in the static case, the OLS residuals $\hat{e}_{t}$ and $\hat{v}_{t}$ under the null are given by (A.7) and (A.8) and the limit of the OLS estimates $\hat{\alpha}$ and $\hat{\beta}$ are given by

$$
\begin{aligned}
\hat{\alpha} & \Rightarrow\left\{\sigma_{e}^{2}+\int_{0}^{1} J^{\prime} \Omega^{\prime} Q \Omega J-\int_{0}^{1} J^{\prime} \Omega^{\prime} Q_{1}^{\prime} Q^{-1} Q_{1} \Omega \int_{0}^{1} J\right\}^{-1}\left\{\int_{0}^{1} J^{\prime} \Omega^{\prime} Q_{1} \Omega J-\int_{0}^{1} J^{\prime} \Omega^{\prime} Q_{1} \Omega \int_{0}^{1} J\right\} \\
& \equiv \bar{\alpha}
\end{aligned}
$$

and $\hat{\beta} \Rightarrow Q^{-1}\left[Q-\bar{\alpha} Q_{1}\right] \Omega \int_{0}^{1} J$. Consider first the $q \hat{L} L$ test. The components of (A.6) will have the following limits (again, $T^{-1} A_{3, T} \stackrel{p}{\rightarrow} 0_{k \times k}$ ):

$$
\begin{aligned}
\hat{V}_{X}= & T^{-1} \sum_{t=1}^{T} \hat{v}_{t} \hat{v}_{t}^{\prime} \\
\Rightarrow & \left(1+\bar{\alpha}^{2}\right) \sigma_{e}^{2} Q+M \Omega\left[\int_{0}^{1} J J^{\prime}-\int_{0}^{1} J \int_{0}^{1} J^{\prime}\right] \Omega^{\prime} M^{\prime}+\bar{\alpha}^{2} M_{11} \Omega\left[\int_{0}^{1} J J^{\prime}\right] \Omega^{\prime} M_{11}^{\prime} \\
\equiv & \bar{V}_{X}=O_{p}\left(\|\Omega\|^{2}\right), \\
T^{-1} A_{1, T}= & T^{-3} \sum_{t=1}^{T}\left[\sum_{s=1}^{t-1} \bar{r}^{s-1} \hat{v}_{t-s}\right]\left[\sum_{s=1}^{t-1} \bar{r}^{s-1} \hat{v}_{t-s}\right]^{\prime} \\
\Rightarrow & {\left[Q-\alpha Q_{1}\right] \Omega \int_{0}^{1}\left[\int_{0}^{r} e^{-c \varsigma} J(r-\varsigma) d \varsigma\right]\left[\int_{0}^{r} e^{-c \varsigma} J(r-\varsigma) d \varsigma\right]^{\prime} d r \Omega^{\prime}\left[Q-\alpha Q_{1}\right]^{\prime} } \\
& +\left[Q-\alpha Q_{1}\right] \Omega \int_{0}^{1} \int_{0}^{r} e^{-c \varsigma} d \varsigma^{2} d r \int_{0}^{1} J \int_{0}^{1} J^{\prime} \Omega^{\prime}\left[Q-\alpha Q_{1}\right]^{\prime} \\
& -\left[Q-\alpha Q_{1}\right] \Omega \int_{0}^{1} \int_{0}^{r} e^{-c \varsigma} d \varsigma \int_{0}^{r} e^{-c \varsigma} J(r-\varsigma) d \varsigma d r \int_{0}^{1} J^{\prime} \Omega^{\prime}\left[Q-\alpha Q_{1}\right]^{\prime} \\
& -\left[Q-\alpha Q_{1}\right] \Omega \int_{0}^{1} J \int_{0}^{1} \int_{0}^{r} e^{-c \varsigma} d \varsigma \int_{0}^{r} e^{-c \varsigma} J(r-\varsigma) d \varsigma d r^{\prime} \Omega^{\prime}\left[Q-\alpha Q_{1}\right]^{\prime}=O_{p}\left(\|\Omega\|^{2}\right),
\end{aligned}
$$




$$
\begin{aligned}
T^{-1} A_{2, T}= & T^{-2} \sum_{t=1}^{T} \hat{v}_{t}\left[\sum_{s=1}^{t-1} \bar{r}^{s-1} \hat{v}_{t-s}\right]^{\prime} \\
\Rightarrow & {\left[Q-\alpha Q_{1}\right] \Omega \int_{0}^{1} J(r) \int_{0}^{r} e^{-c \varsigma} J(r-\varsigma) d \varsigma d r \Omega\left[Q-\alpha Q_{1}\right]^{\prime} } \\
& \quad-\left[Q-\alpha Q_{1}\right] \Omega \int_{0}^{1} \int_{0}^{r} e^{-c \varsigma} d \varsigma J(r) d r \int_{0}^{1} J^{\prime} \Omega^{\prime}\left[Q-\alpha Q_{1}\right]^{\prime} \\
& \quad\left[Q-\alpha Q_{1}\right] \Omega \int_{0}^{1} J \int_{0}^{1} \int_{0}^{r} e^{-c \varsigma} J(r-\varsigma) d \varsigma d r^{\prime} \Omega^{\prime}\left[Q-\alpha Q_{1}\right]^{\prime} \\
& +\left[Q-\alpha Q_{1}\right] Q \Omega \int_{0}^{1} \int_{0}^{r} e^{-c \varsigma} d \varsigma d r \int_{0}^{1} J \int_{0}^{1} J^{\prime} \Omega^{\prime}\left[Q-\alpha Q_{1}\right]^{\prime}=O_{p}\left(\|\Omega\|^{2}\right), \\
T^{-1 / 2} A_{4, T}= & T^{-1} \sum_{t=1}^{T} \bar{r}^{t} \hat{v}_{t} \Rightarrow\left[Q-\alpha Q_{1}\right] \Omega \int_{0}^{1} e^{-c r} J-\left[Q-\alpha Q_{1}\right] \Omega \int_{0}^{1} e^{-c r} \int_{0}^{1} J=O_{p}(\|\Omega\|), \\
T^{-1 / 2} A_{5, T}= & T^{-2} \sum_{t=1}^{T} \bar{r}^{t} \sum_{s=1}^{t-1} \bar{r}^{s-1} \hat{v}_{t-s} \\
\Rightarrow & {\left[Q-\alpha Q_{1}\right] \Omega \int_{0}^{1} e^{-c r} \int_{0}^{r} e^{-c \varsigma} J(r-\varsigma) d \varsigma d r-[Q-\alpha Q 1] \Omega \int_{0}^{1} e^{-c r} \int_{0}^{r} e^{-c \varsigma} d \varsigma d r \int_{0}^{1} J } \\
= & O_{p}(\|\Omega\|) .
\end{aligned}
$$

Collecting terms yields the stated results for the $q \hat{L} L$ test. For the sup-Wald test, the restricted sum of squared residuals is such that

$$
\begin{aligned}
T^{-1} S S R_{T}^{r}= & T^{-1} \sum_{t=1}^{T}\left[e_{t}-\hat{\alpha} e_{t-1}+X_{t}^{\prime} \beta_{t}-\hat{\alpha} X_{t-1}^{\prime} \beta_{t-1}-X_{t} \hat{\beta}\right]^{2} \\
\Rightarrow & \left(1+\bar{\alpha}^{2}\right) \sigma_{e}^{2}+\left(1+\bar{\alpha}^{2}\right) \int_{0}^{1} J^{\prime} \Omega^{\prime} Q \Omega J-2 \bar{\alpha} \int_{0}^{1} J^{\prime} \Omega^{\prime} Q_{1} \Omega J \\
& \quad-\int_{0}^{1} J^{\prime} \Omega^{\prime}\left[Q-\bar{\alpha} Q_{1}\right]^{\prime} Q^{-1}\left[Q-\bar{\alpha} Q_{1}\right] \Omega \int_{0}^{1} J \equiv S S R^{r *}=O_{p}\left(\|\Omega\|^{2}\right),
\end{aligned}
$$

where $\bar{\alpha}$ is given by (A.16). For the unrestricted regression the residuals are

$$
\hat{e}(\lambda)_{t}=e_{t}+X_{t}^{\prime} \beta_{t}-\hat{\alpha}_{u} e_{t-1}-\hat{\alpha}_{u} X_{t-1}^{\prime} \beta_{t-1}-X_{1}(\lambda)_{t}^{\prime} \hat{\beta}_{1}-X_{2}(\lambda)_{t}^{\prime} \hat{\beta}_{2}
$$

where the estimates of the coefficients have the following limits:

$$
\begin{aligned}
\hat{\alpha}_{u} \Rightarrow & \left\{\sigma_{e}^{2}+\int_{0}^{1} J^{\prime} \Omega^{\prime} Q \Omega J-\frac{1}{\lambda} \int_{0}^{\lambda} J^{\prime} \Omega^{\prime} Q_{1}^{\prime} Q^{-1} Q_{1} \Omega \int_{0}^{\lambda} J-\frac{1}{1-\lambda} \int_{\lambda}^{1} J^{\prime} \Omega^{\prime} Q_{1}^{\prime} Q^{-1} Q_{1} \Omega \int_{\lambda}^{1} J\right\}^{-1} \\
& \times\left\{\int_{0}^{1} J^{\prime} \Omega^{\prime} Q_{1} \Omega J-\frac{1}{\lambda} \int_{0}^{\lambda} J^{\prime} \Omega^{\prime} Q_{1} \Omega \int_{0}^{\lambda} J-\frac{1}{1-\lambda}\left[\int_{\lambda}^{1} J\right]^{\prime} \Omega^{\prime} Q_{1} \Omega\left[\int_{\lambda}^{1} J\right]\right\} \equiv \bar{\alpha}_{u}, \quad(\mathrm{~A} .17) \\
T^{-1 / 2} \hat{\beta}_{1} \Rightarrow & (1 / \lambda) \Omega Q^{-1}\left[Q-\bar{\alpha}_{u} Q_{1}\right] \int_{0}^{\lambda} J \text { and } T^{-1 / 2} \hat{\beta}_{2} \Rightarrow(1 /(1-\lambda)) \Omega Q^{-1}\left[Q-\bar{\alpha}_{u} Q_{1}\right] \int_{\lambda}^{1} J .
\end{aligned}
$$

Therefore, the limit of $S S R(\lambda)_{T}$ is given by

$$
\begin{aligned}
T^{-1} S S R(\lambda)_{T}= & T^{-2} \sum_{t=1}^{T}\left[e_{t}+X_{t}^{\prime} \beta_{t}-\hat{\alpha}_{u} e_{t-1}-\hat{\alpha}_{u} X_{t-1}^{\prime} \beta_{t-1}-X_{1}(\lambda)_{t}^{\prime} \hat{\beta}_{1}-X_{2}(\lambda)_{t}^{\prime} \hat{\beta}_{2}\right]^{2} \\
\Rightarrow & \left(1+\bar{\alpha}_{u}^{2}\right) \sigma_{e}^{2}+\left(1+\bar{\alpha}_{u}^{2}\right) \int_{0}^{1} J^{\prime} \Omega^{\prime} Q \Omega J-2 \bar{\alpha}_{u} \int_{0}^{1} J^{\prime} \Omega^{\prime} Q_{1} \Omega J \\
& -\frac{1}{\lambda} \int_{0}^{\lambda} J^{\prime} \Omega^{\prime}\left[Q-\bar{\alpha}_{u} Q_{1}\right]^{\prime} Q^{-1}\left[Q-\bar{\alpha}_{u} Q_{1}\right] \Omega \int_{0}^{\lambda} J \\
& -\frac{1}{1-\lambda} \int_{\lambda}^{1} J^{\prime} \Omega^{\prime}\left[Q-\bar{\alpha}_{u} Q_{1}\right]^{\prime} Q^{-1}\left[Q-\bar{\alpha}_{u} Q_{1}\right] \Omega \int_{\lambda}^{1} J \equiv S S R(\lambda)^{*}=O_{p}\left(\|\Omega\|^{2}\right),
\end{aligned}
$$


where $\bar{\alpha}_{u}$ is given by (A.17). By the continuous mapping theorem, the limit of the sup-Wald test is given by

$$
T^{-1} S W=\left(\frac{T^{-1} S S R_{T}^{r}-T^{-1} S S R_{T}\left(\bar{\lambda}_{T}\right)}{T^{-1} S S R_{T}\left(\bar{\lambda}_{T}\right)}\right) \Rightarrow\left(\frac{S S R^{r *}-S S R^{*}(\bar{\lambda})}{S S R^{*}(\bar{\lambda})}\right)=O_{p}(1),
$$

where $\bar{\lambda}_{T}$ is the value of $\lambda$ that minimizes $S S R_{T}(\lambda)$ and $\bar{\lambda}$ minimizes $S S R^{*}(\lambda)$.

Proof of Theorem 7 (single break parameter; i.i.d. errors assumed): The OLS estimate of $\beta$ now has the following limit:

$$
\hat{\beta}=\left[T^{-1} \sum_{t=1}^{T} X_{t} X_{t}^{\prime}\right]^{-1}\left[T^{-1} \sum_{t=1}^{T} X_{t} X_{t}^{\prime} \beta_{t}+T^{-1} \sum_{t=1}^{T} X_{t} e_{t}\right] \stackrel{p}{\rightarrow}\left(1-\lambda_{c}\right) \theta
$$

and $\hat{e}_{t}$ and $\hat{v}_{t}$ are still given by (A.7) and (A.8). We now consider the limits of $\hat{V}_{X}$ and $A_{i, T}$, $i=1, \ldots, 5$ (again, $T^{-1} A_{3, T} \stackrel{p}{\rightarrow} 0_{k \times k}$ ).

$$
\begin{aligned}
& \hat{V}_{X}=T^{-1} \sum_{t=1}^{T} \hat{v}_{t} \hat{v}_{t}^{\prime} \stackrel{p}{\rightarrow} \sigma_{e}^{2} Q+\lambda_{c}\left(1-\lambda_{c}\right) M \theta \theta^{\prime} M^{\prime} \equiv \bar{V}_{X}=O\left(\|\theta\|^{2}\right), \\
& T^{-1} A_{1, T}=T^{-3} \sum_{t=1}^{T}\left[\sum_{s=1}^{t-1} \bar{r}^{s-1} \hat{v}_{t-s}\right]\left[\sum_{s=1}^{t-1} \bar{r}^{s-1} \hat{v}_{t-s}\right]^{\prime} \\
& \stackrel{p}{\rightarrow} \int_{\lambda_{c}}^{1}\left(\int_{0}^{r-\lambda_{c}} e^{-c \varsigma} d \varsigma\right)^{2} d r Q \theta \theta^{\prime} Q^{\prime}+\left(1-\lambda_{c}\right)^{2} \int_{0}^{1}\left(\int_{0}^{r} e^{-c \varsigma} d \varsigma\right)^{2} d r Q \theta \theta^{\prime} Q^{\prime} \\
& -2\left(1-\lambda_{c}\right) \int_{\lambda_{c}}^{1}\left(\int_{0}^{r-\lambda_{c}} e^{-c \varsigma} d \varsigma\right)\left(\int_{0}^{r} e^{-c \varsigma} d \varsigma\right) d r Q \theta \theta^{\prime} Q^{\prime}=O\left(\|\theta\|^{2}\right), \\
& T^{-1} A_{2, T}=T^{-2} \sum_{t=1}^{T} \hat{v}_{t}\left[\sum_{s=1}^{t-1} \bar{r}^{s-1} \hat{v}_{t-s}\right]^{\prime} \\
& \stackrel{p}{\rightarrow} \int_{\lambda_{c}}^{1}\left(\int_{0}^{r-\lambda_{c}} e^{-c \varsigma} d \varsigma\right) d r Q \theta \theta^{\prime} Q^{\prime}+\left(1-\lambda_{c}\right)^{2} \int_{0}^{1} \int_{0}^{r} e^{-c \varsigma} d \varsigma d r Q \theta \theta^{\prime} Q^{\prime} \\
& -\left(1-\lambda_{c}\right) \int_{\lambda_{c}}^{1}\left(\int_{0}^{r} e^{-c \varsigma} d \varsigma\right) d r Q \theta \theta^{\prime} Q^{\prime}-\left(1-\lambda_{c}\right) \int_{\lambda_{c}}^{1} \int_{0}^{r-\lambda_{c}} e^{-c \varsigma} d \varsigma d r Q \theta \theta^{\prime} Q^{\prime} \\
& =O\left(\|\theta\|^{2}\right) \text {, } \\
& T^{-1 / 2} A_{4, T}=T^{-1} \sum_{t=1}^{T} \bar{r}^{t} \hat{v}_{t} \stackrel{p}{\rightarrow} \int_{\lambda_{c}}^{1} e^{-c r} d r Q \theta-\left(1-\lambda_{c}\right) \int_{0}^{1} e^{-c r} d r Q \theta=O(\|\theta\|), \\
& T^{-1 / 2} A_{5, T}=T^{-2} \sum_{t=1}^{T} \bar{r}^{t} \sum_{s=1}^{t-1} \bar{r}^{s-1} \hat{v}_{t-s} \\
& \stackrel{p}{\rightarrow} \int_{\lambda_{c}}^{1} e^{-c r} \int_{0}^{r-\lambda_{c}} e^{-c \varsigma} d \varsigma d r Q \theta-\left(1-\lambda_{c}\right) \int_{0}^{1} e^{-c r} \int_{0}^{r} e^{-c \varsigma} d \varsigma d r Q \theta=O(\|\theta\|) .
\end{aligned}
$$


Collecting terms and using the representation (A.6) yields the following limit for the $q \hat{L} L$ statistic:

$$
T^{-1} q \hat{L} L \stackrel{p}{\rightarrow} \xi \operatorname{tr}\left\{\bar{V}_{X}^{-1 / 2} Q \theta \theta^{\prime} Q^{\prime} \bar{V}^{\prime-1 / 2}\right\},
$$

where

$$
\begin{aligned}
\xi= & \lambda_{c}\left(\lambda_{c}-1\right)+\frac{1}{c}\left(1-\lambda_{c}+\frac{1}{2} \lambda_{c}^{2}\right)-\frac{1}{c}\left(1-\lambda_{c}\right)^{2} e^{-c}-\frac{1}{2 c}\left(1-\lambda_{c}\right)^{2} e^{-2 c}-\frac{1}{c}\left(1-\lambda_{c}\right) e^{-c \lambda_{c}} \\
& +\frac{1}{c}\left(1-\lambda_{c}\right) e^{c\left(\lambda_{c}-2\right)}-\frac{1}{2 c} e^{2 c\left(\lambda_{c}-1\right)}-\frac{1}{2 c\left(1-e^{-2 c}\right)}\left[\lambda_{c}-1+\left(1-\lambda_{c}\right) e^{-2 c}+e^{-c \lambda_{c}}-e^{c\left(\lambda_{c}-2\right)}\right]^{2} .
\end{aligned}
$$

For the sup-Wald test, the sum of squared restricted residuals is such that

$$
T^{-1} S S R_{T}^{r}=T^{-1} \sum_{t=1}^{T}\left[e_{t}+X_{t}^{\prime} \theta_{t}-X_{t}^{\prime} \hat{\beta}\right]^{2} \stackrel{p}{\rightarrow} \sigma_{e}^{2}+\lambda_{c}\left(1-\lambda_{c}\right) \theta^{\prime} Q \theta
$$

and we can show that $T^{-1} S S R_{T}(\lambda) \stackrel{p}{\rightarrow} \sigma_{e}^{2}$. Hence, by the continuous mapping theorem

$$
T^{-1} S W=\left(\frac{T^{-1} S S R_{T}^{r}-T^{-1} S S R_{T}\left(\bar{\lambda}_{T}\right)}{T^{-1} S S R_{T}\left(\bar{\lambda}_{T}\right)}\right) \stackrel{p}{\rightarrow} \frac{\lambda_{c}\left(1-\lambda_{c}\right)}{\sigma_{e}^{2}} \theta^{\prime} Q \theta
$$

where $\bar{\lambda}_{T}$ minimizes $S S R_{T}(\lambda)$.

Proof of Theorem 8 (single break parameter; i.i.d. correction for serial correlation): The $q \hat{L} L$ test uses the restricted residuals so that the autocovariances $\hat{\Gamma}_{j}$ are such that

$$
\begin{aligned}
\hat{\Gamma}_{j}= & T^{-1} \sum_{t=j+1}^{T} X_{t} e_{t} e_{t-j} X_{t-j}^{\prime}+T^{-1} \sum_{t=j+1}^{T} X_{t} X_{t}^{\prime} \beta_{t} \beta_{t-j}^{\prime} X_{t-j}^{\prime} X_{t-j}+T^{-1} \sum_{t=j+1}^{T} X_{t} X_{t}^{\prime} \hat{\beta} \hat{\beta}^{\prime} X_{t-j}^{\prime} X_{t-j} \\
& -T^{-1} \sum_{t=j+1}^{T} X_{t} X_{t}^{\prime} \beta_{t} \hat{\beta}^{\prime} X_{t-j}^{\prime} X_{t-j}-T^{-1} \sum_{t=j+1}^{T} X_{t} X_{t}^{\prime} \hat{\beta} \beta_{t-j}^{\prime} X_{t-j}^{\prime} X_{t-j}+o_{p}(1) \\
& \stackrel{p}{\rightarrow} \lambda_{c}\left(1-\lambda_{c}\right) M_{j j} \theta \theta^{\prime} M_{j j}^{\prime}=O\left(\|\theta\|^{2}\right) .
\end{aligned}
$$

The bandwidth involves $\bar{\alpha}$ defined by (A.15) where

$$
\hat{\rho}_{i} \stackrel{p}{\rightarrow} \frac{\lambda_{c}\left(1-\lambda_{c}\right)\left\{M_{11}\right\}_{i i}^{2}\left\{\theta \theta^{\prime}\right\}_{i i}}{\sigma_{e}^{2}\{Q\}_{i i}+\lambda_{c}\left(1-\lambda_{c}\right)\{M\}_{i i}^{2}\left\{\theta \theta^{\prime}\right\}_{i i}} \equiv \bar{\rho}_{i}
$$

and $\bar{\sigma}_{v i}^{4}$ is bounded for the same reason as Theorem 2. In $\bar{\rho}_{i}$, the term $\sigma_{e}^{2}\{Q\}_{i i}$ is dominated as $\|\theta\|$ increases, and if $\left\{M-M_{11}\right\}_{i i}=0, \hat{\rho}_{i}$ converges to 1 at rate $\|\theta\|^{2}$. Hence, $\alpha=O_{p}\left(\|\theta\|^{4}\right)$ and $m=O_{p}\left(\|\theta\|^{4 / 3} T^{1 / 3}\right)$. This yields $\hat{h}(0)=O_{p}\left(\|\theta\|^{10 / 3} T^{1 / 3}\right)$. Since $\hat{V}_{X}=O_{p}\left(\|\theta\|^{2}\right)$, the result follows. The sup-Wald test uses unrestricted residuals in the construction of $\hat{h}_{l}(0)$. Now, it is straightforward to show that when $\lambda=\lambda_{c}, \hat{\Gamma}_{j} \stackrel{p}{\rightarrow} 0$ for $j \neq 0$, since $E\left(e_{t} e_{t-j}\right)=0$ 
if $j \neq 0$. For the bandwidth, since $\hat{\rho}_{l, i} \stackrel{p}{\rightarrow} 0, \alpha_{l}=O_{p}(1)$, and $m=O_{p}\left(T^{1 / 3}\right)$, so that $\hat{h}_{l}(0)=O_{p}\left(T^{1 / 3}\right)$ for $l=1,2$ and the result follows.

Proof of Theorem 9 (single break parameter; lagged dependent variable): For the model under the null hypothesis, the OLS residuals $\hat{e}_{t}$ and $\hat{v}_{t}$ are given by (A.10) and (A.11) and the OLS estimates $\hat{\alpha}$ and $\hat{\beta}$ have the following limits:

$$
\hat{\alpha} \stackrel{p}{\rightarrow}\left[\sigma_{e}^{2}+\left(1-\lambda_{c}\right) \theta^{\prime} Q \theta-\left(1-\lambda_{c}\right)^{2} \theta^{\prime} Q_{1} Q^{-1} Q_{1} \theta\right]^{-1}\left[\lambda_{c}\left(1-\lambda_{c}\right) \theta^{\prime} Q_{1} \theta\right] \equiv \bar{\alpha}
$$

and

$$
\hat{\beta} \stackrel{p}{\rightarrow}\left(1-\lambda_{c}\right) \theta-\bar{\alpha}\left(1-\lambda_{c}\right) Q^{-1} Q_{1} \theta .
$$

For the $q \hat{L} L$ test, the components of (A.6) have the following limits (again, $T^{-1} A_{3, T} \stackrel{p}{\rightarrow} 0_{k \times k}$ ):

$$
\begin{gathered}
\hat{V}_{X}=T^{-1} \sum_{t=1}^{T} \hat{v}_{t} \hat{v}_{t}^{\prime} \stackrel{p}{\rightarrow}\left(1+\bar{\alpha}^{2}\right) \sigma_{e}^{2} Q+\left(1-\lambda_{c}\right) \lambda_{c} M \theta \theta^{\prime} M^{\prime}+\bar{\alpha}^{2}\left(1-\lambda_{c}\right) M_{11} \theta \theta^{\prime} M_{11}^{\prime} \\
-2 \bar{\alpha}\left(1-\lambda_{c}\right) \lambda_{c} M_{1} \theta \theta^{\prime} M_{1}^{\prime}+\bar{\alpha}^{2}\left(1-\lambda_{c}\right)^{2} M Q^{-1} Q_{1} \theta \theta^{\prime} Q_{1}^{\prime} Q^{-1 \prime} M^{\prime} \\
-\bar{\alpha}^{2}\left(1-\lambda_{c}\right)^{2} M_{1}\left[Q^{-1} Q_{1} \theta \theta^{\prime}+\theta \theta^{\prime} Q_{1}^{\prime} Q^{-1 \prime}\right] M_{1}^{\prime} \equiv \bar{V}_{X}=O\left(\|\Omega\|^{2}\right), \\
T^{-1} A_{1, T}=T^{-3} \sum_{t=1}^{T}\left[\sum_{s=1}^{t-1} \bar{r}^{s-1} \hat{v}_{t-s}\right]\left[\sum_{s=1}^{t-1} \bar{r}^{s-1} \hat{v}_{t-s}\right]^{\prime} \stackrel{p}{\rightarrow} \int_{\lambda_{c}}^{1}\left(\int_{0}^{r-\lambda_{c}} e^{-c s} d s\right)^{2} d r\left[Q-\bar{\alpha} Q_{1}\right] \theta \theta^{\prime}\left[Q-\bar{\alpha} Q_{1}\right]^{\prime} \\
+\left(1-\lambda_{c}\right)^{2} \int_{0}^{1}\left(\int_{0}^{r} e^{-c s} d s\right)^{2} d r\left[Q-\bar{\alpha} Q_{1}\right] \theta \theta^{\prime}\left[Q-\bar{\alpha} Q_{1}\right]^{\prime} \\
-2\left(1-\lambda_{c}\right) \int_{\lambda_{c}}^{1} \int_{0}^{r-\lambda_{c}} e^{-c s} d s \int_{0}^{r} e^{-c s} d s d r\left[Q-\bar{\alpha} Q_{1}\right] \theta \theta^{\prime}\left[Q-\bar{\alpha} Q_{1}\right]^{\prime}=O\left(\|\theta\|^{2}\right), \\
T^{-1} A_{2, T}=T^{-2} \sum_{t=1}^{T} \hat{v}_{t}\left[\sum_{s=1}^{t-1} \bar{r}^{s-1} \hat{v}_{t-s}\right]^{\prime} \stackrel{p}{\rightarrow} \lambda_{c} \int_{\lambda_{c}}^{1} \int_{0}^{r-\lambda_{c}} e^{-c s} d s d r\left[Q-\bar{\alpha} Q_{1}\right] \theta \theta^{\prime}\left[Q-\bar{\alpha} Q_{1}\right]^{\prime} \\
-\lambda_{c}\left(1-\lambda_{c}\right) \int_{\lambda_{c}}^{1} \int_{0}^{r} e^{-c s} d s d r\left[Q-\bar{\alpha} Q_{1}\right] \theta \theta^{\prime}\left[Q-\bar{\alpha} Q_{1}\right]^{\prime} \\
-\lambda_{c}\left(1-\lambda_{c}\right) \int_{\lambda_{c}}^{1} \int_{0}^{r-\lambda_{c}} e^{-c s} d s d r\left[Q-\bar{\alpha} Q_{1}\right] \theta \theta^{\prime}\left[Q-\bar{\alpha} Q_{1}\right]^{\prime}=O\left(\|\theta\|^{2}\right), \\
T^{-1 / 2} A_{4, T}=T^{-1} \sum_{t=1}^{T} \bar{r}^{t} \hat{v}_{t} \stackrel{p}{\rightarrow} \int_{\lambda_{c}}^{1} e^{-c r} d r\left[Q-\bar{\alpha} Q_{1}\right] \theta-\left(1-\lambda_{c}\right) \int_{0}^{1} e^{-c r} d r\left[Q-\bar{\alpha} Q_{1}\right] \theta=O(\|\theta\|), \\
T^{-1 / 2} A_{5, T} \quad=T^{-2} \sum_{t=1}^{T} \bar{r}^{t} \sum_{s=1}^{t-1} \bar{r}^{s-1} \hat{v}_{t-s} \stackrel{p}{\rightarrow} \int_{\lambda_{c}}^{1} e^{-c r} \int_{0}^{r-\lambda_{c}} e^{-c s} d s d r\left[Q-\bar{\alpha} Q_{1}\right] \theta \\
\quad-\left(1-\lambda_{c}\right) \int_{0}^{1} e^{-c r} \int_{0}^{r-\lambda_{c}} e^{-c s} d s d r\left[Q-\bar{\alpha} Q_{1}\right] \theta=O(\|\theta\|) .
\end{gathered}
$$

Then solving the integrals and collecting terms using (A.6) gives the result of the $q \hat{L} L$ test. For the sup-Wald test, the restricted sum of squared residuals is such that

$$
\begin{aligned}
T^{-1} S S R_{T}^{r}= & T^{-1} \sum_{t=1}^{T} \hat{e}_{t}^{2} \stackrel{p}{\rightarrow}\left(1+\bar{\alpha}^{2}\right) \sigma_{e}^{2}+\left(1-\lambda_{c}\right)\left(1+\bar{\alpha}^{2}\right) \theta^{\prime} Q \theta-2\left(1-\lambda_{c}\right) \bar{\alpha} \theta^{\prime} Q_{1} \theta \\
& -\left(1-\lambda_{c}\right)^{2} \theta^{\prime}\left[Q-\bar{\alpha} Q_{1}\right]^{\prime} Q^{-1}\left[Q-\bar{\alpha} Q_{1}\right] \theta \equiv S S R^{r *}
\end{aligned}
$$


where $\bar{\alpha}$ is given by (A.18). For the unrestricted regression

$$
y_{t}=\alpha_{u} y_{t-1}+X_{1}(\lambda)_{t}^{\prime} \beta_{1}+X_{2}(\lambda)_{t}^{\prime} \beta_{2}+e_{t}
$$

we can show that for $\lambda \leq \lambda_{c}$,

$$
\begin{aligned}
& \left.\hat{\alpha}_{u} \stackrel{p}{\rightarrow}\left[\sigma_{e}^{2}+\left(1-\lambda_{c}\right) \theta^{\prime} Q \theta-\frac{\left(1-\lambda_{c}\right)^{2}}{1-\lambda} \theta^{\prime} Q_{1}^{\prime} Q^{-1} Q_{1} \theta\right]^{-1}\left[1-\lambda_{c}\right) \theta^{\prime} Q_{1} \theta-\frac{\left(1-\lambda_{c}\right)^{2}}{1-\lambda} \theta^{\prime} Q_{1}^{\prime} \theta\right] \equiv \bar{\alpha}_{u}, \\
& \hat{\beta}_{1} \stackrel{p}{\rightarrow} 0_{k \times 1}, \\
& \hat{\beta}_{2} \stackrel{p}{\rightarrow} \frac{1-\lambda_{c}}{1-\lambda} Q^{-1}\left[Q-\bar{\alpha}_{u} Q_{1}\right] \theta .
\end{aligned}
$$

For $\lambda>\lambda_{c}$,

$$
\begin{aligned}
& \hat{\alpha}_{u} \stackrel{p}{\rightarrow}\left[\sigma_{e}^{2}+\left(1-\lambda_{c}\right) \theta^{\prime} Q \theta-\frac{\left(\lambda-\lambda_{c}\right)^{2}+\lambda(1-\lambda)}{\lambda} \theta^{\prime} Q_{1}^{\prime} Q^{-1} Q_{1} \theta\right]^{-1} \\
& \times\left[\left(1-\lambda_{c}\right) \theta^{\prime} Q_{1} \theta-\frac{\left(\lambda-\lambda_{c}\right)^{2}+\lambda(1-\lambda)}{\lambda} \theta^{\prime} Q_{1}^{\prime} \theta\right] \\
& \hat{\beta}_{1} \stackrel{p}{\rightarrow} \frac{\lambda-\lambda_{c}}{\lambda} Q^{-1}\left[Q-\bar{\alpha}_{u} Q_{1}\right] \theta \\
& \hat{\beta}_{2} \stackrel{p}{\rightarrow} Q^{-1}\left[Q-\bar{\alpha}_{u} Q_{1}\right] \theta
\end{aligned}
$$

More importantly, for $\lambda=\lambda_{c}, \hat{\alpha}_{u} \stackrel{p}{\rightarrow} 0, \hat{\beta}_{1} \stackrel{p}{\rightarrow} 0_{k \times 1}$, and $\hat{\beta}_{2} \stackrel{p}{\rightarrow} \theta$. Now, $S S R(\lambda)_{T}$ will take a minimum value at $\lambda=\lambda_{c}$ so that

$$
T^{-1} S S R\left(\lambda_{c}\right)_{T}=T^{-1} \sum_{t=1}^{T} \hat{e}\left(\lambda_{c}\right)_{t}^{2}=T^{-1} \sum_{t=1}^{T} e_{t}^{2}+o_{p}(1) \stackrel{p}{\rightarrow} \sigma_{e}^{2}
$$

Therefore, by the continuous mapping theorem,

$$
T^{-1} S W=\left(\frac{T^{-1} S S R_{T}^{r}-T^{-1} S S R_{T}(\bar{\lambda})}{T^{-1} S S R_{T}(\bar{\lambda})}\right) \stackrel{p}{\rightarrow}\left(\frac{S S R^{r^{*}}-\sigma_{e}^{2}}{\sigma_{e}^{2}}\right)=O\left(\|\theta\|^{2}\right),
$$

where $\bar{\lambda}_{T}$ minimizes $S S R_{T}(\lambda)$. For the special case with $X_{t}=\{1\}$ for all $t$, it is clear that $\bar{\alpha}=1$ and $Q-\bar{\alpha} Q_{1}=0$ so that $p \lim _{T \rightarrow \infty} T^{-1} q \hat{L} L=0$. For the sup-Wald test, the limit of $S S R^{r *}$ reduces to $2 \sigma_{e}^{2}$, which implies $p \lim _{T \rightarrow \infty} T^{-1} S W=1$. 
Figure 1: US real interest rate

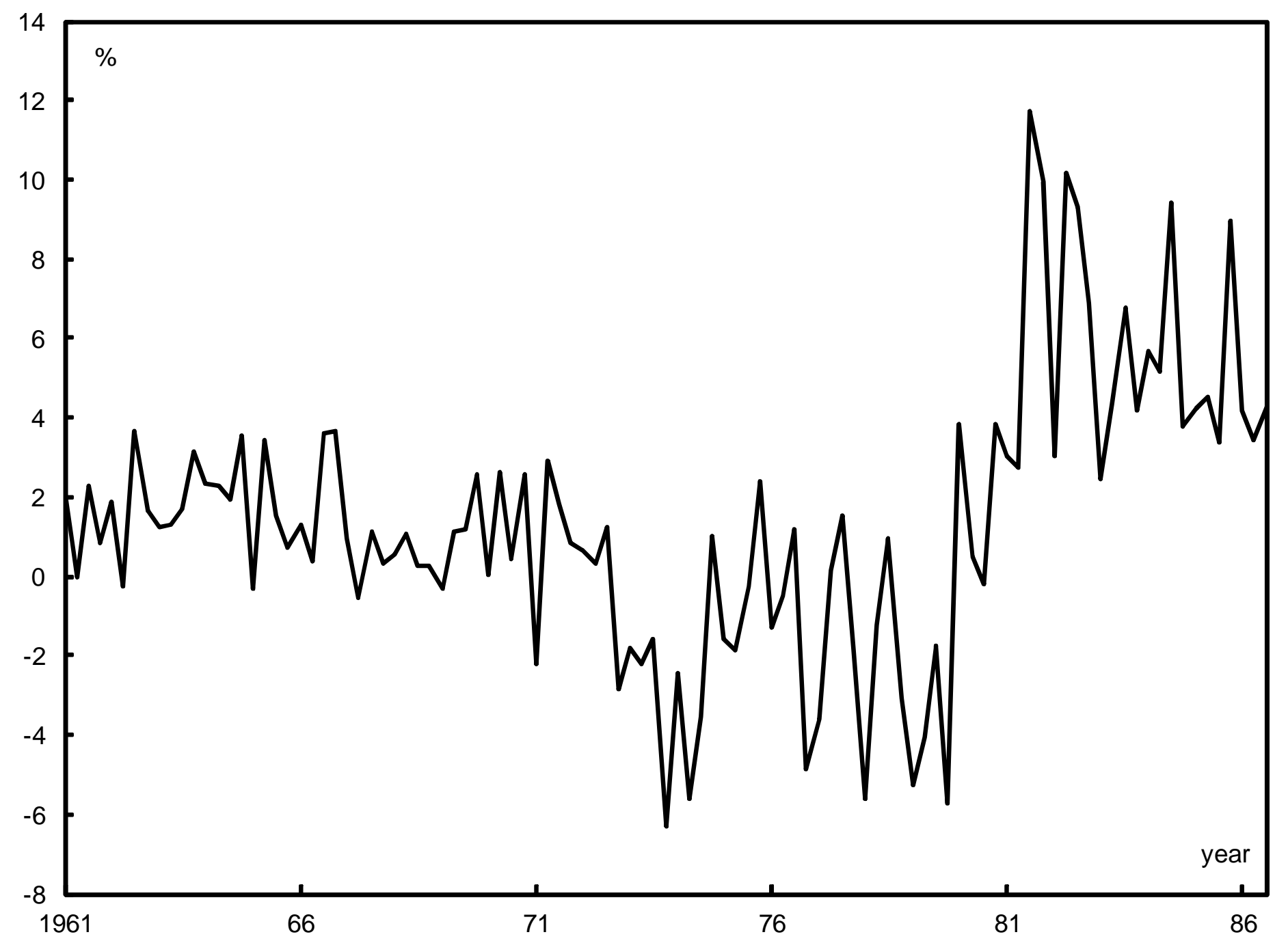


Figure 2: Power functions for Models A, B

Model A

static / non robust

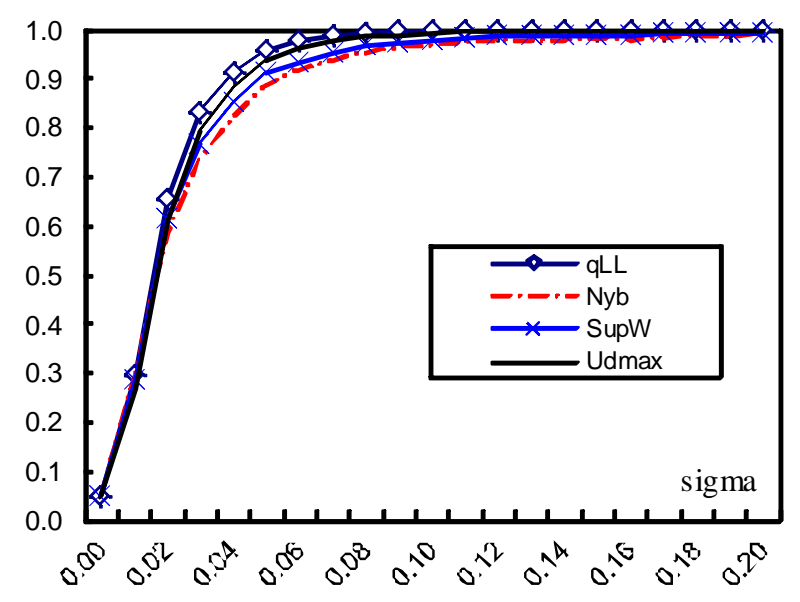

static / robust

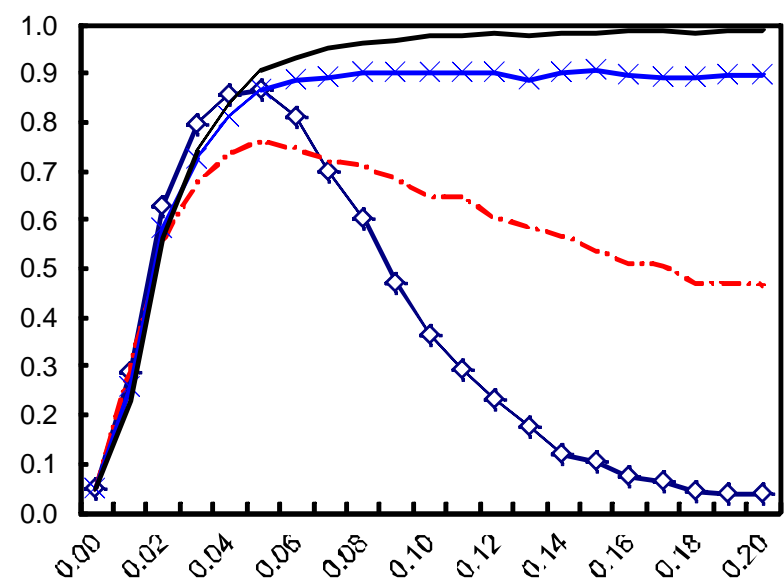

dynamic

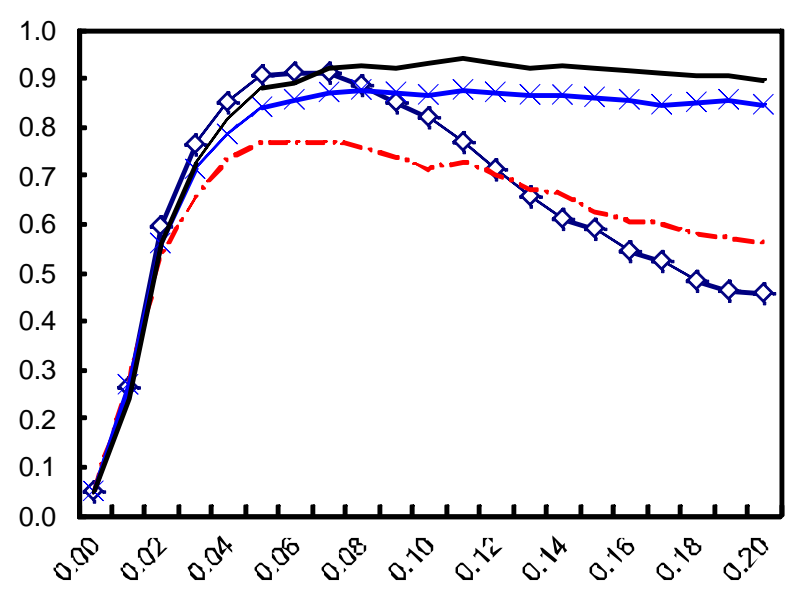

Model B

static / non robust

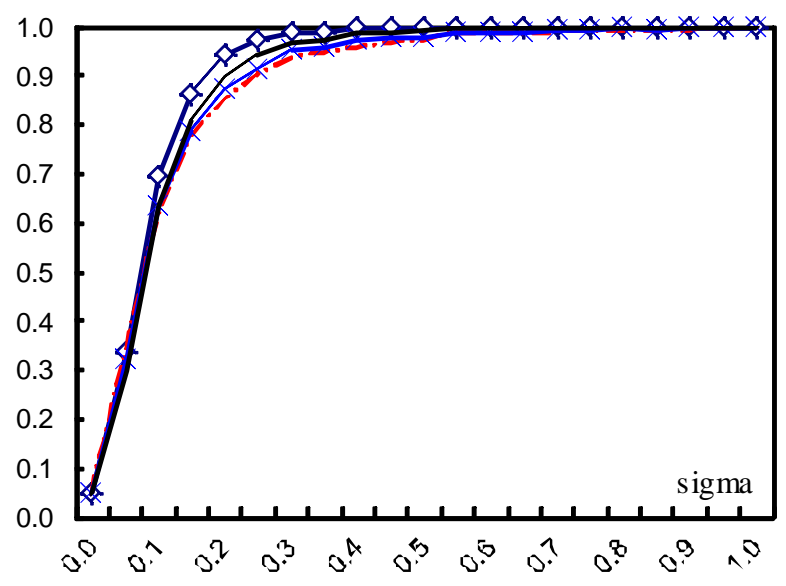

static / robust

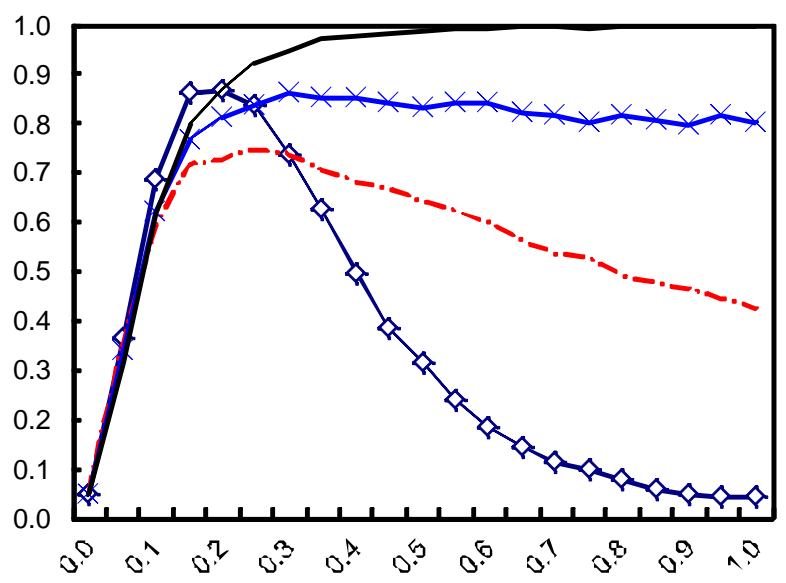

dynamic

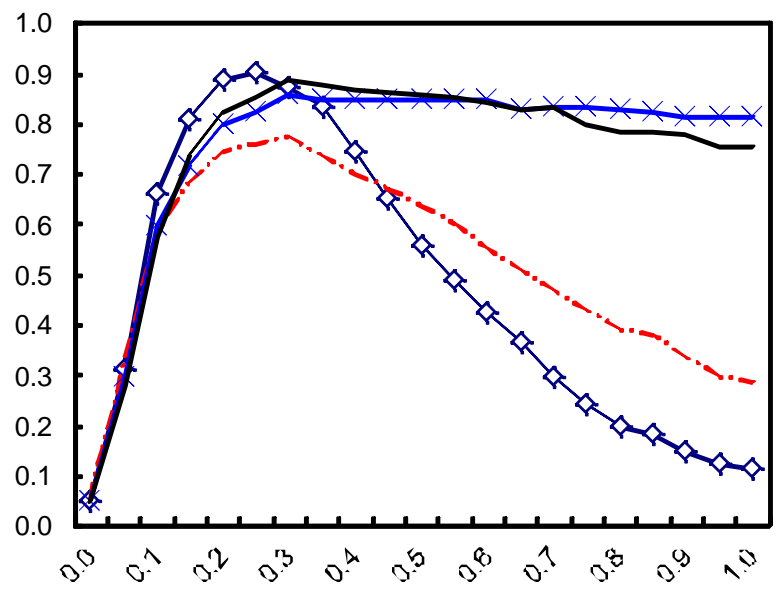


Figure 3: Power functions of Models C, D

Model C

static / non robust

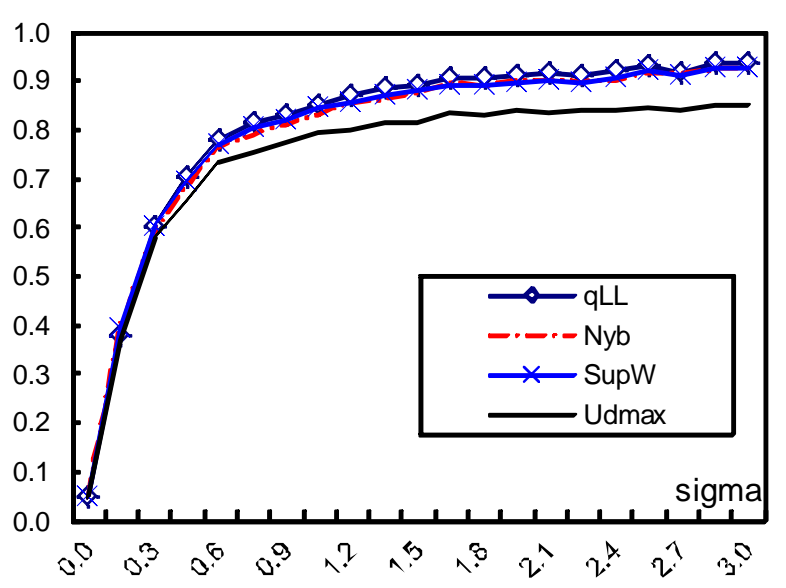

static / robust

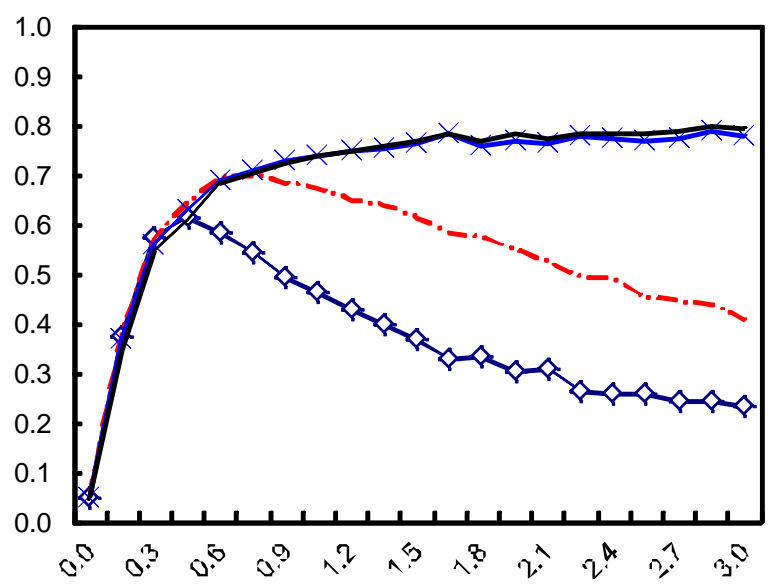

dynamic

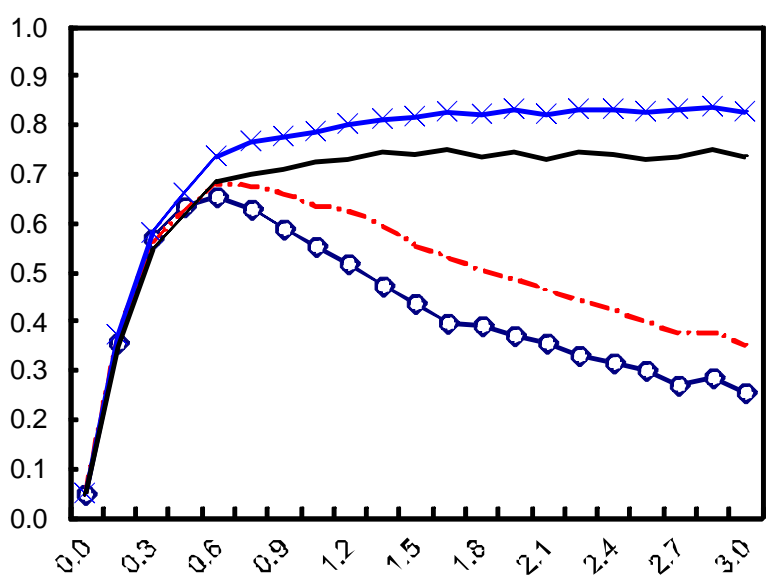

Model D

static / non robust

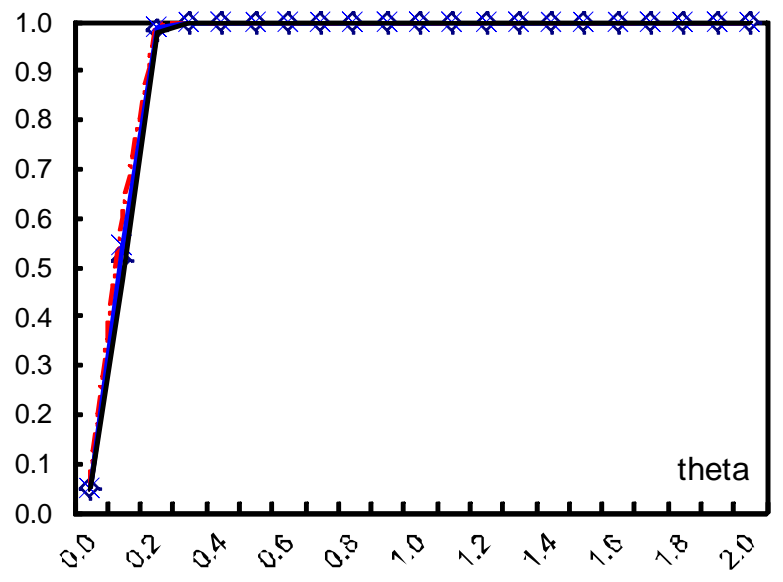

static / robust

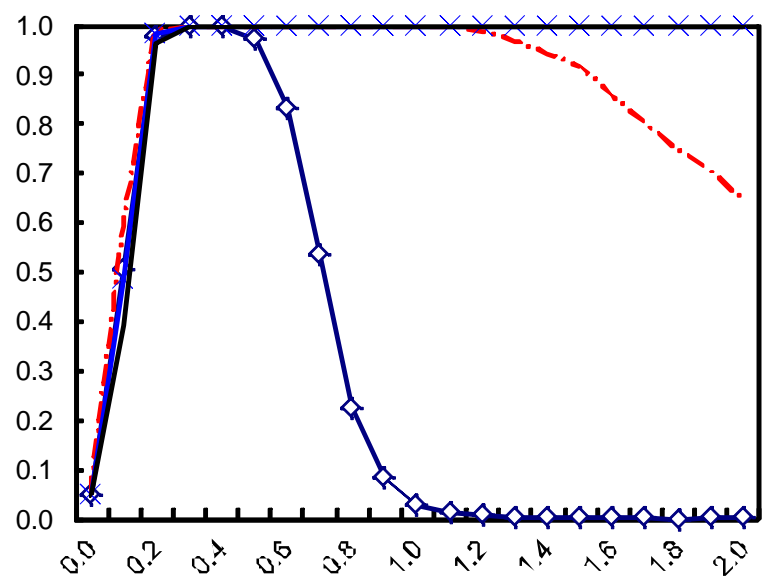

dynamic

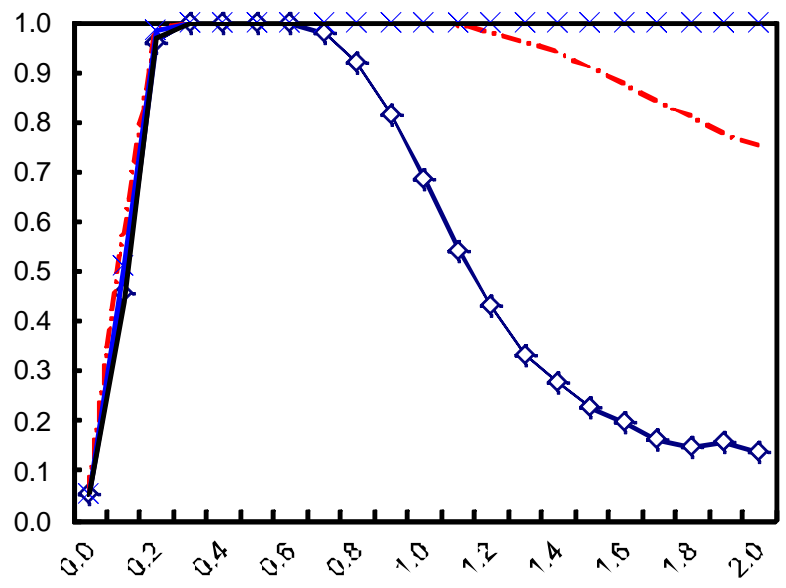


Figure 4 : Hybrid tests, Models A, B

Model A

Power of hybrid test

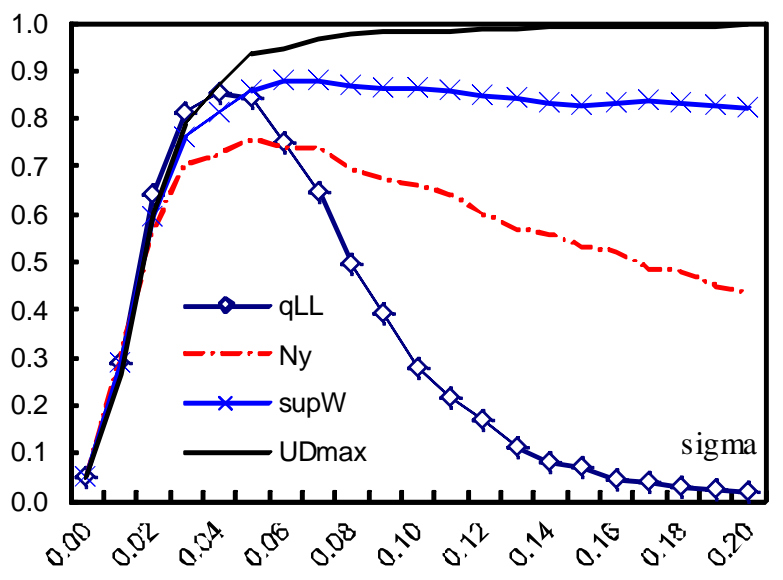

Power of serial correlation test

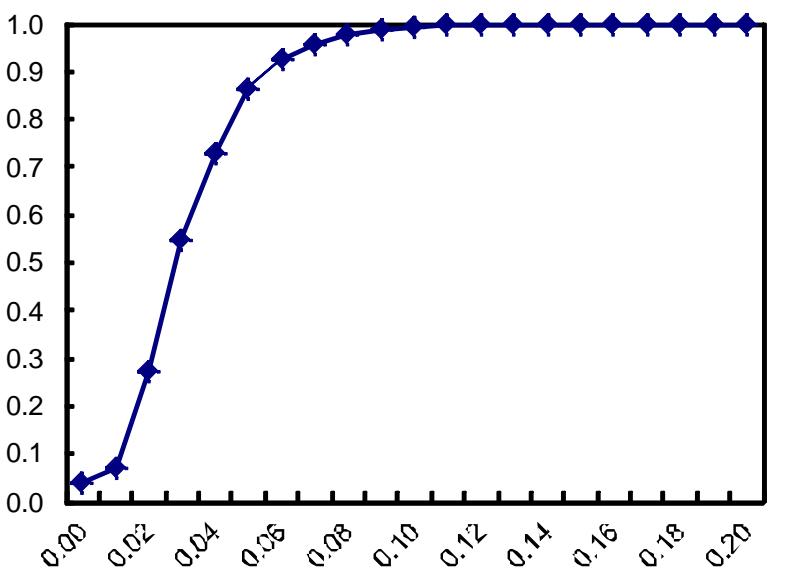

AR1 coefficient (mean value)

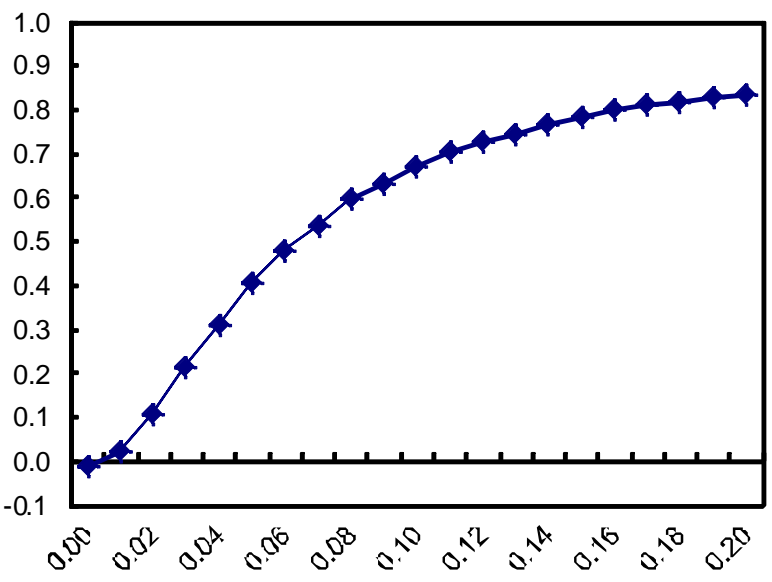

Model B

Power of hybrid test

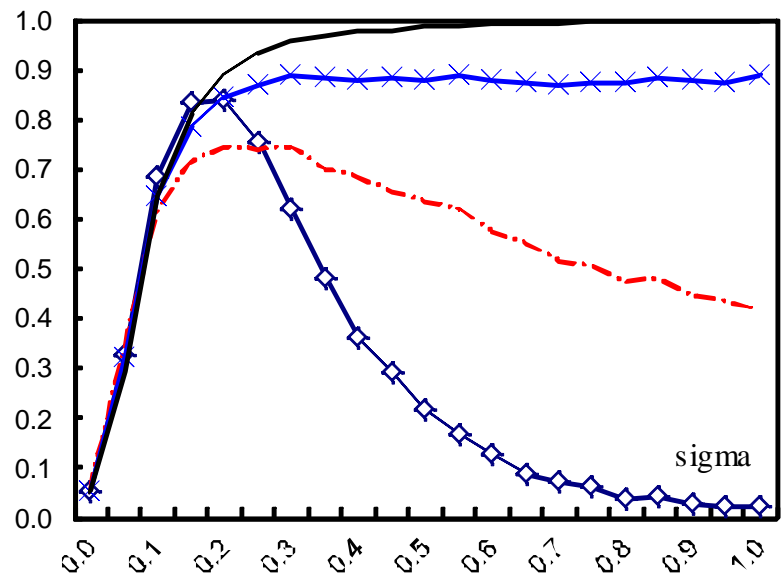

Power of serial correlation test

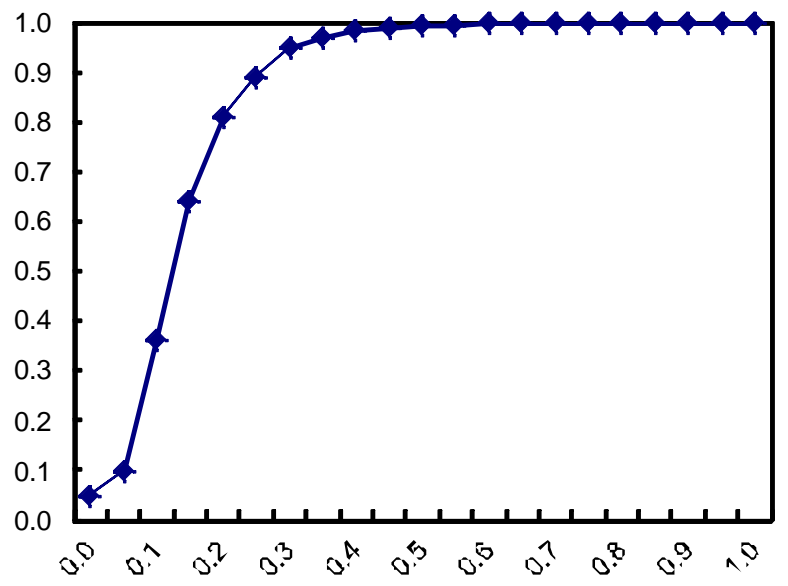

AR1 coefficient (mean value)

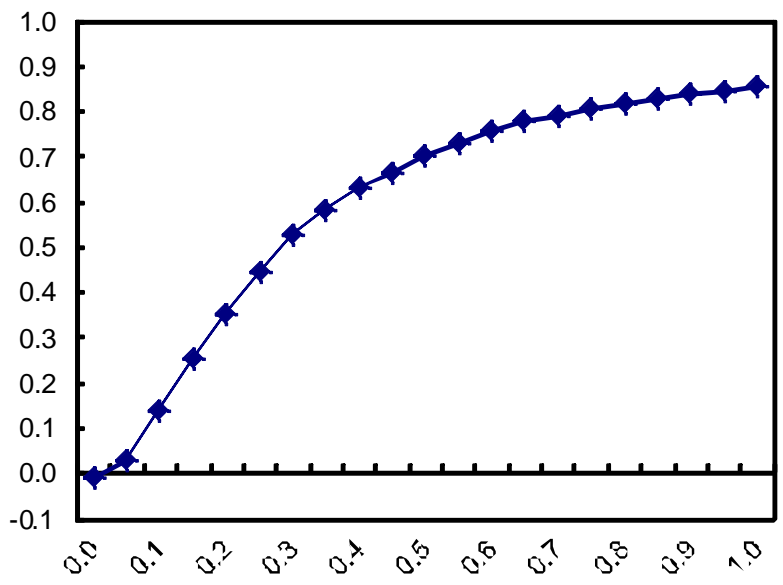


Figure 5 : Hybrid tests, Models C, D

Model C

Power of hybrid test

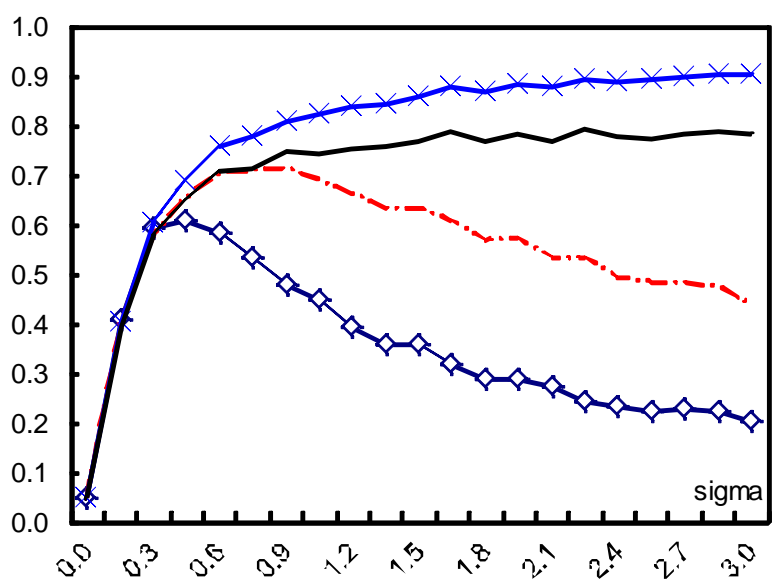

Power of serial correlation test

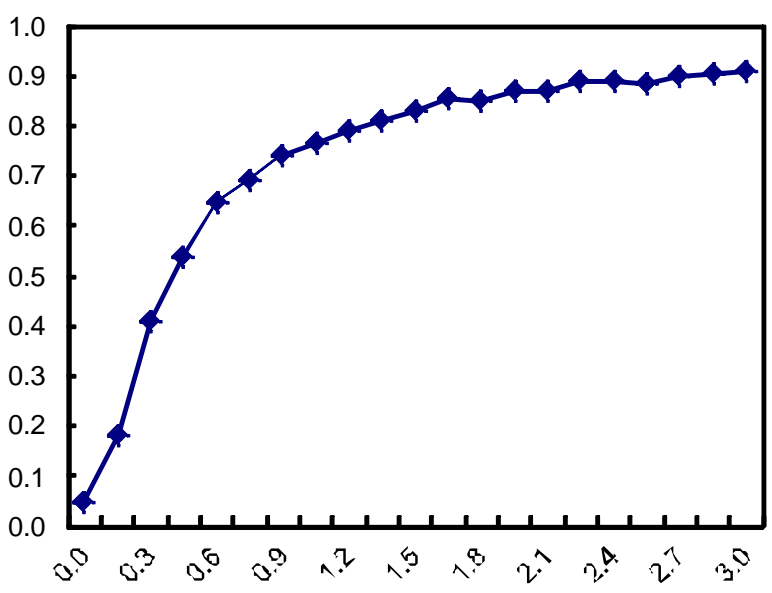

AR1 coefficient (mean value)

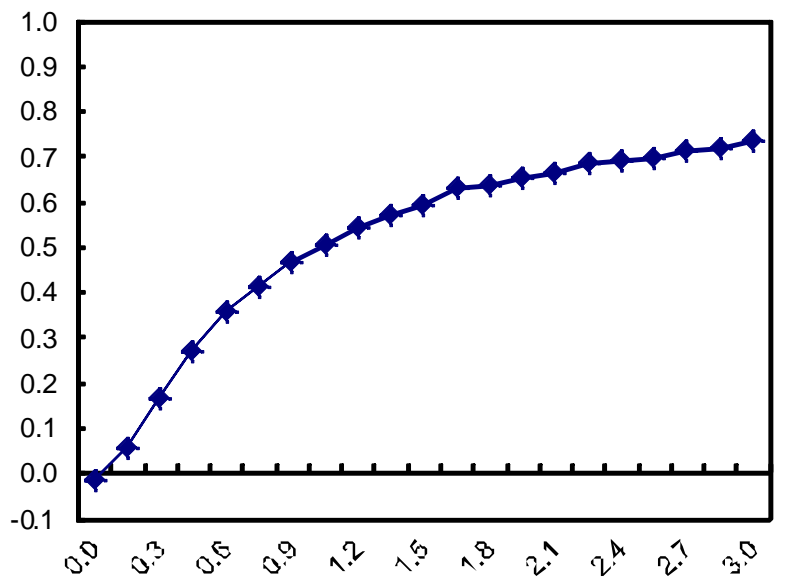

Model D

Power of hybrid test

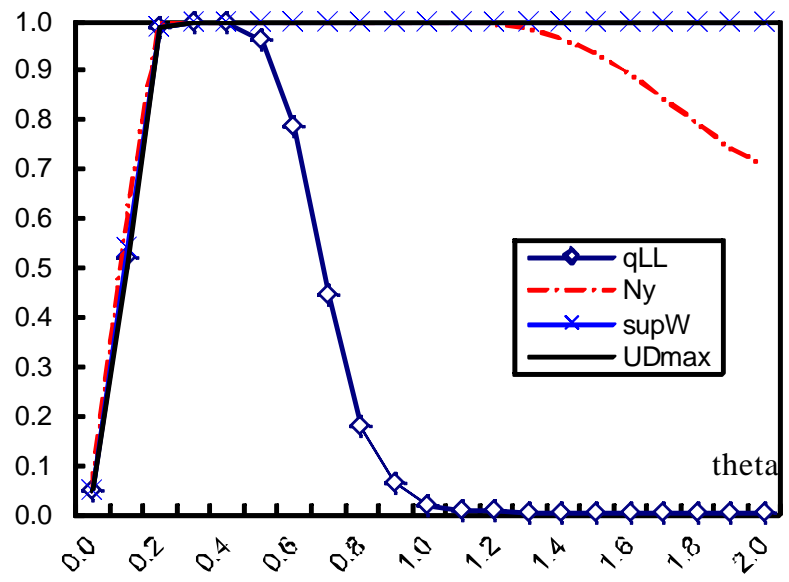

Power of serial correlation test

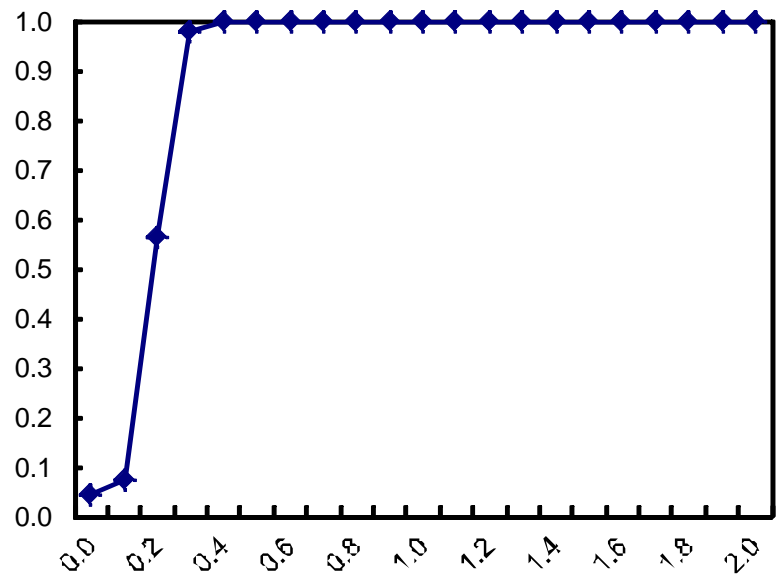

AR1 coefficient (mean value)

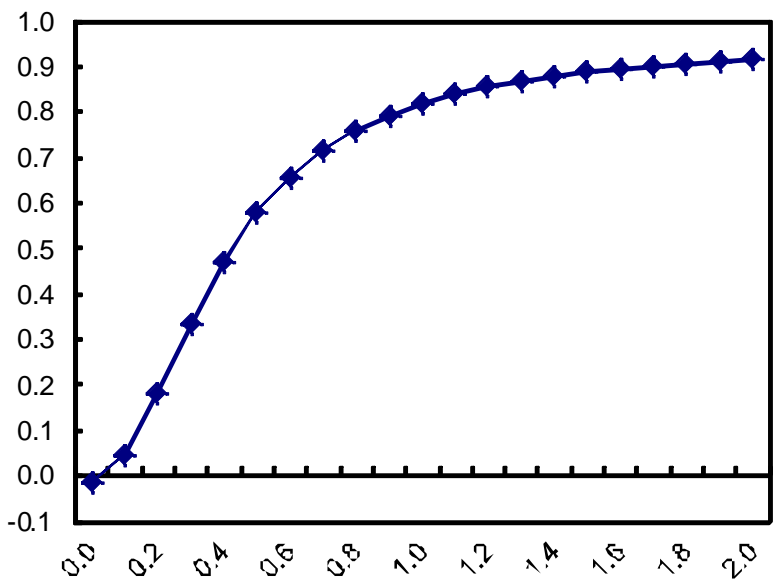


Figure 6 : Limits of $-T^{-2 / 3} q \hat{L} L$ and $T^{-2 / 3} S W$, HAC robust test

Random walk model
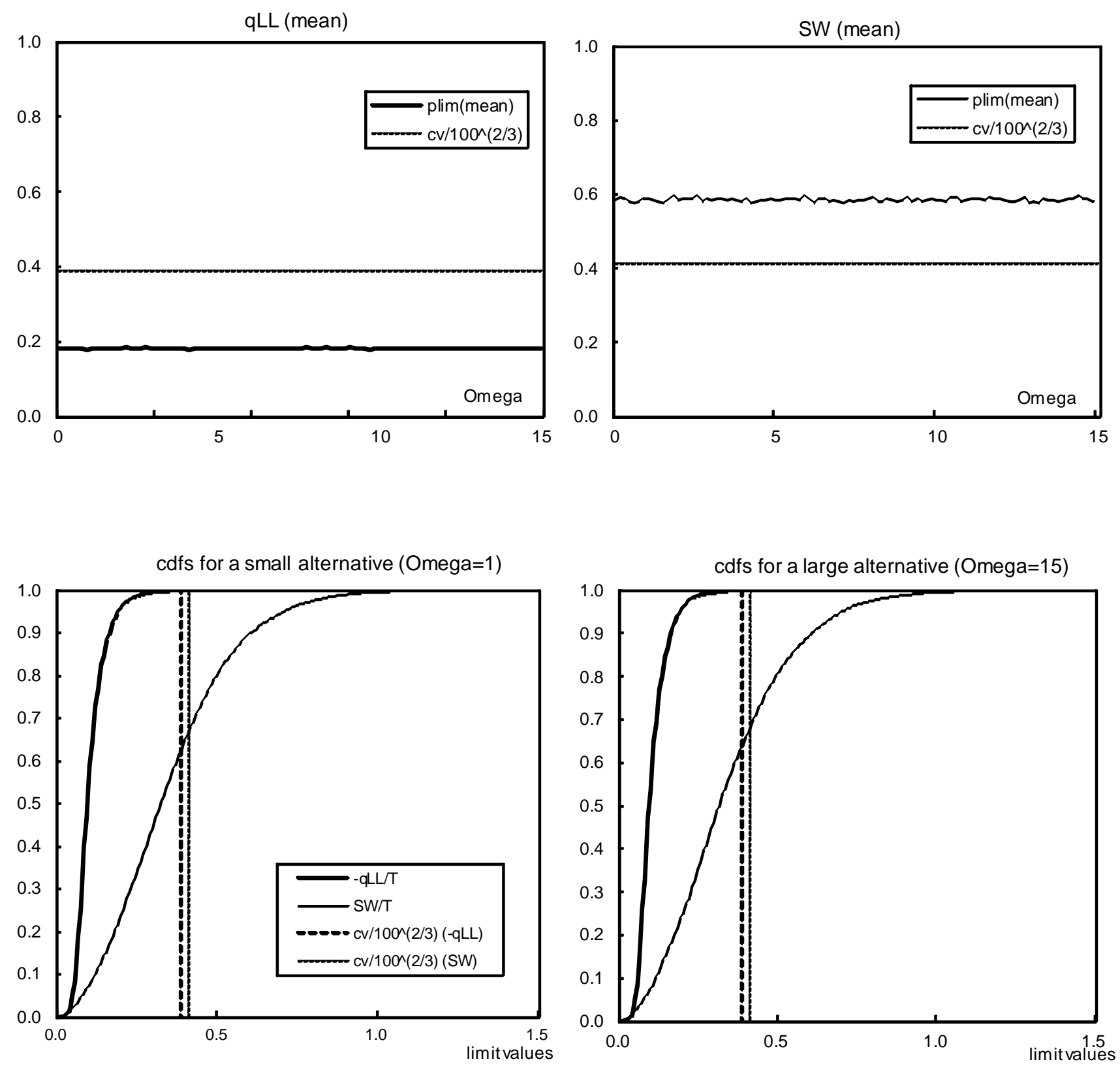
Figure 7 : Limits of $-T^{-1} q \hat{L} L$ and $T^{-1} S W$, dynamic regression

Random walk model
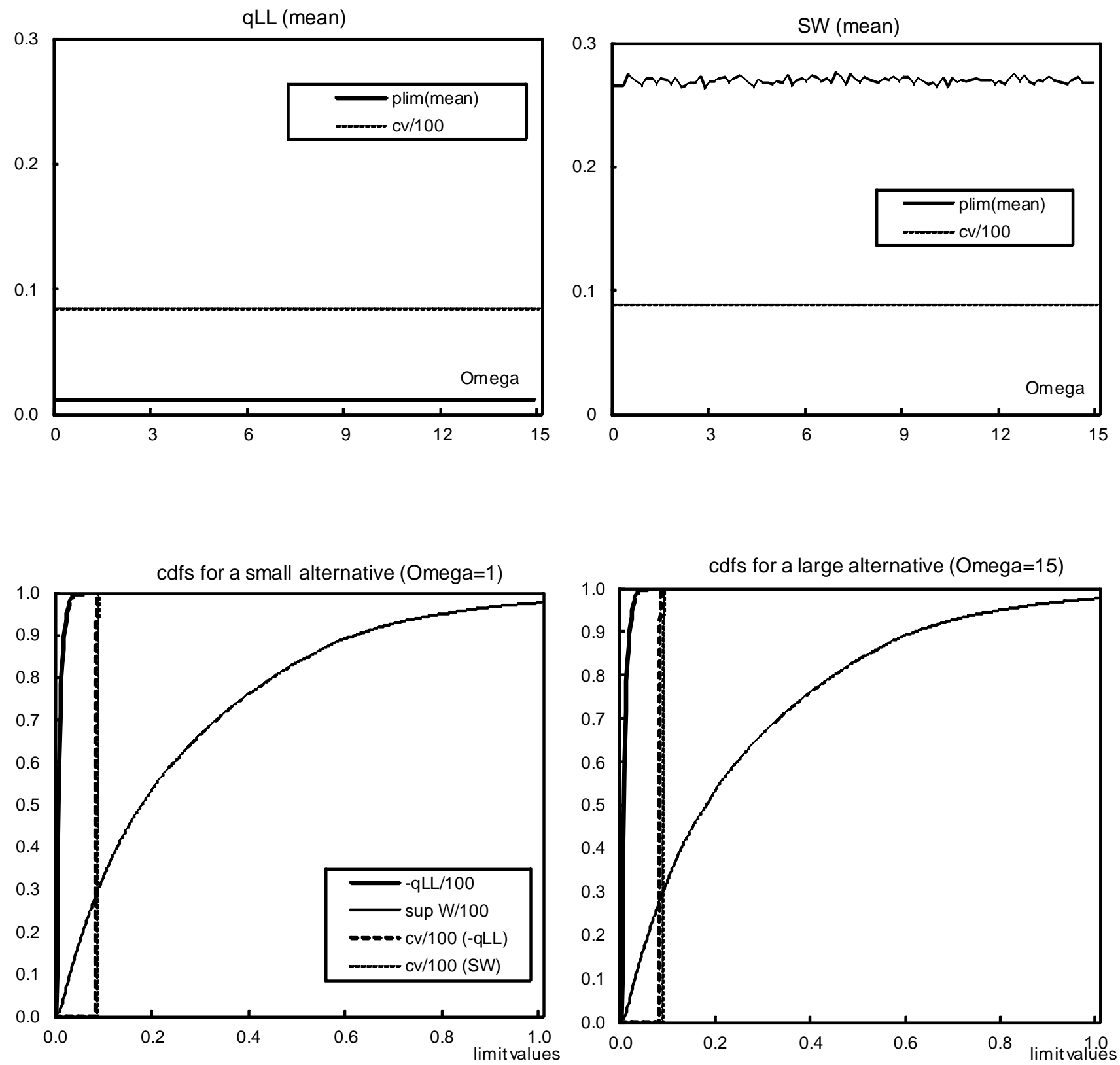
Figure 8a: Limits of $-T^{-1} q \hat{L} L$ and $T^{-1} S W$, non-robust test

Random level shift model with intensity $p / T=0.05$
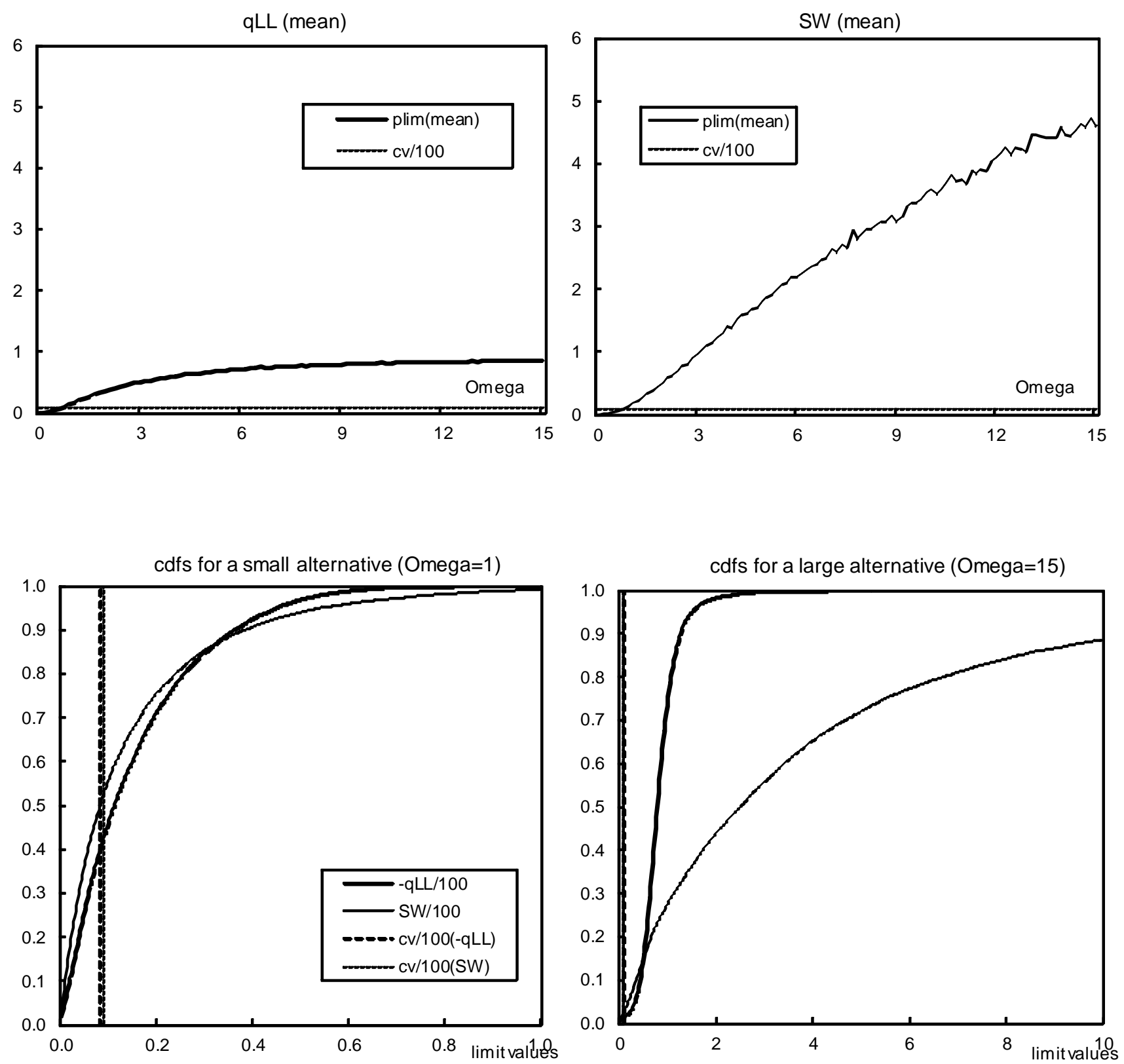
Figure 8b: Limits of $-T^{-1} q \hat{L} L$ and $T^{-1} S W$, non-robust test

Random level shift model with intensity $p / T=0.1$
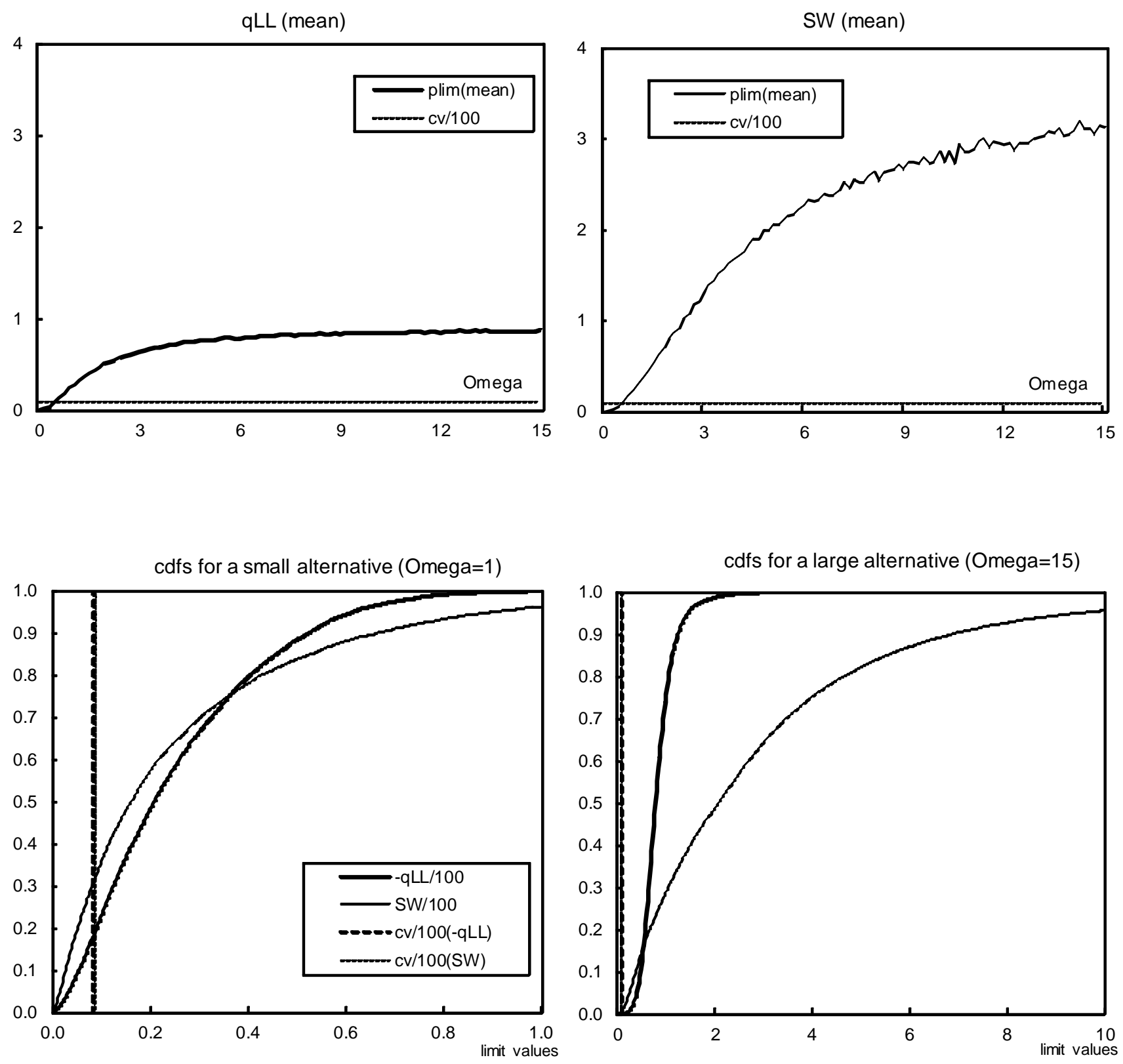
Figure 8c: Limits of $-T^{-1} q \hat{L} L$ and $T^{-1} S W$, non-robust test

Random level shift model with intensity $p / T=0.5$
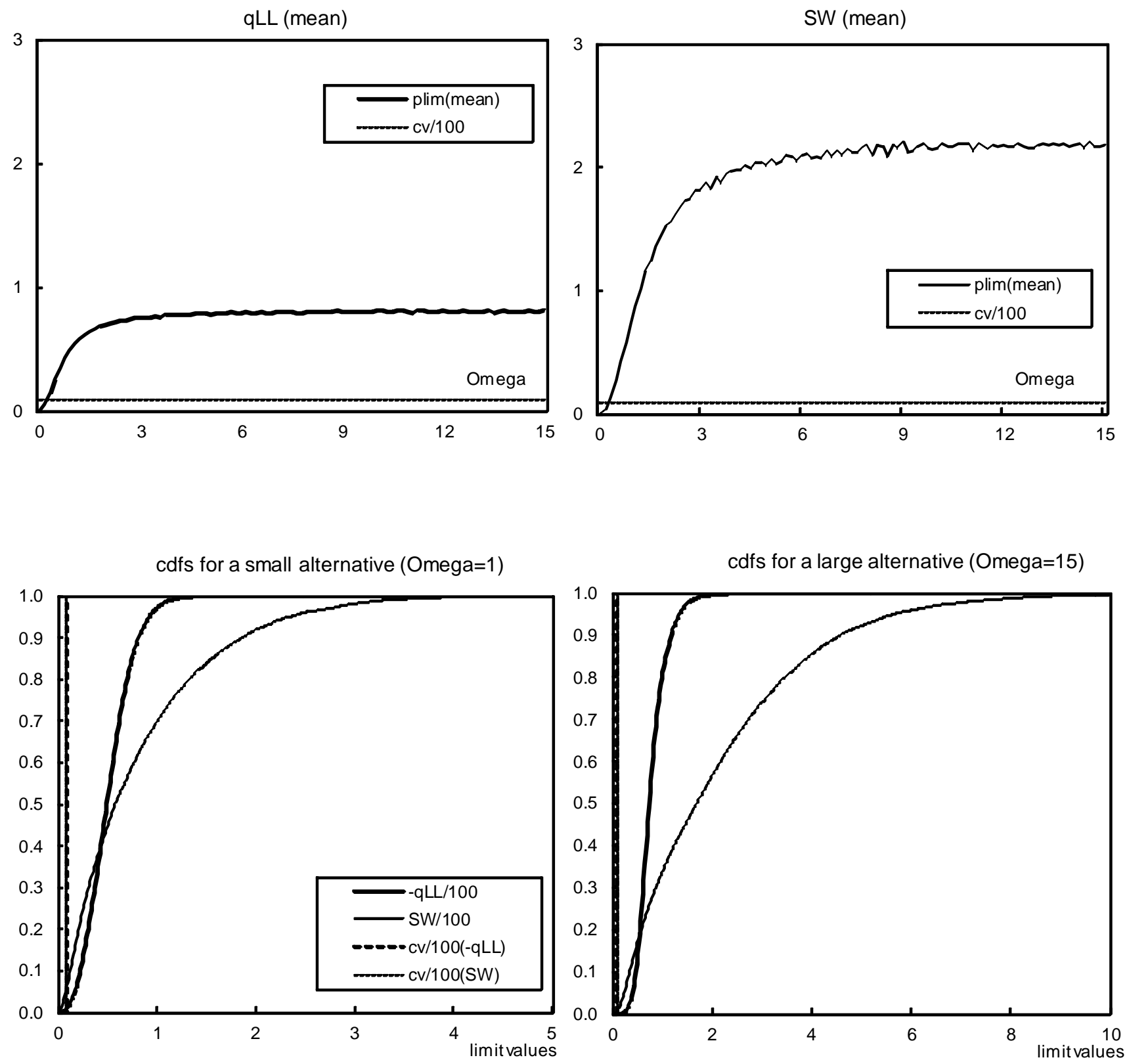
Figure 9a: Limits of $-T^{-2 / 3} q \hat{L} L$ and $T^{-2 / 3} S W$, HAC robust test Random level shift model with intensity $p / T=0.05$
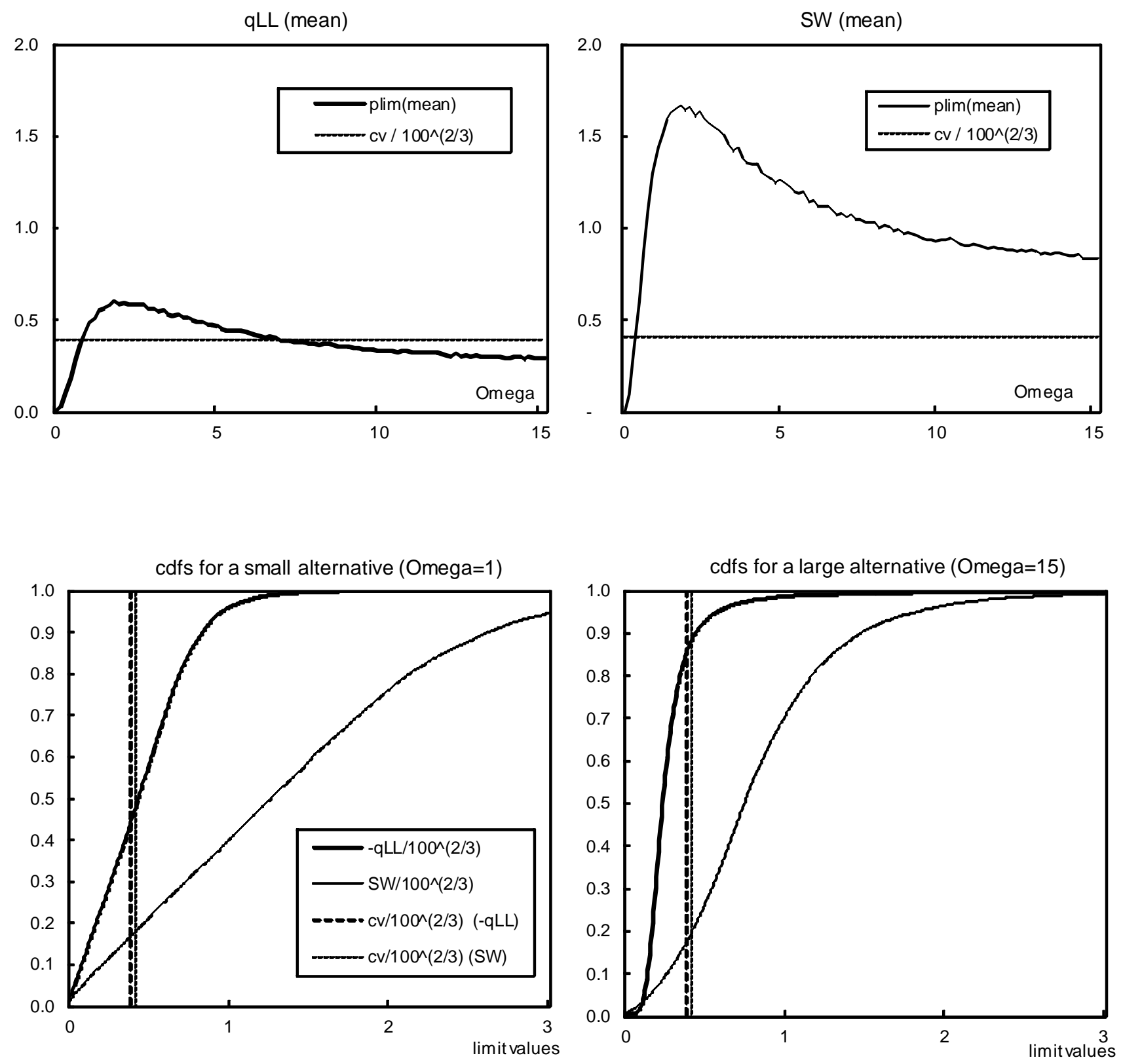
Figure $9 \mathrm{~b}:$ Limits of $-T^{-2 / 3} q \hat{L} L$ and $T^{-2 / 3} S W$, HAC robust test

Random level shift model with intensity $p / T=0.1$
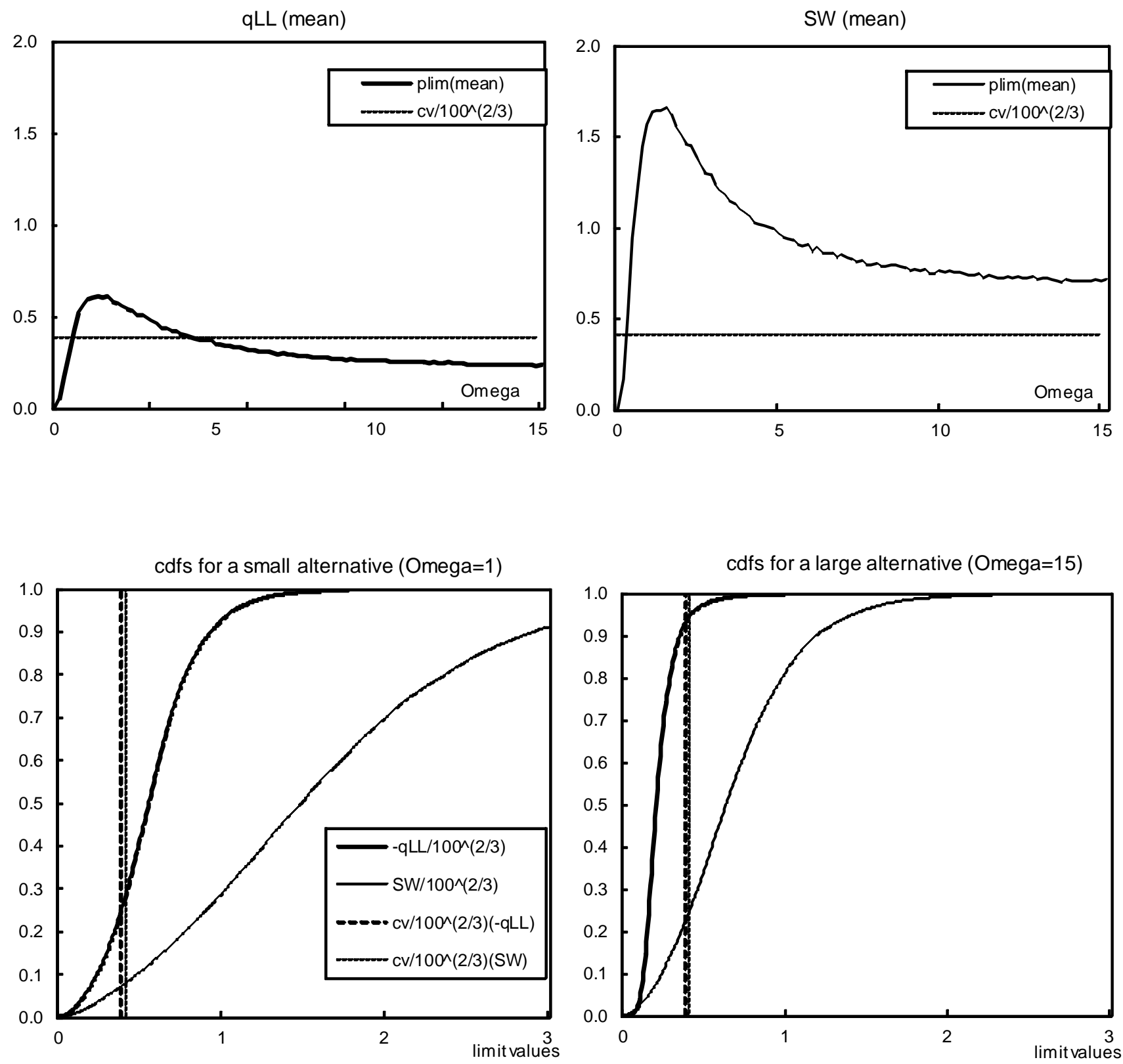
Figure $9 \mathrm{c}$ : Limits of $-T^{-2 / 3} q \hat{L} L$ and $T^{-2 / 3} S W$, HAC robust test,

Random level shift model with intensity $p / T=0.5$
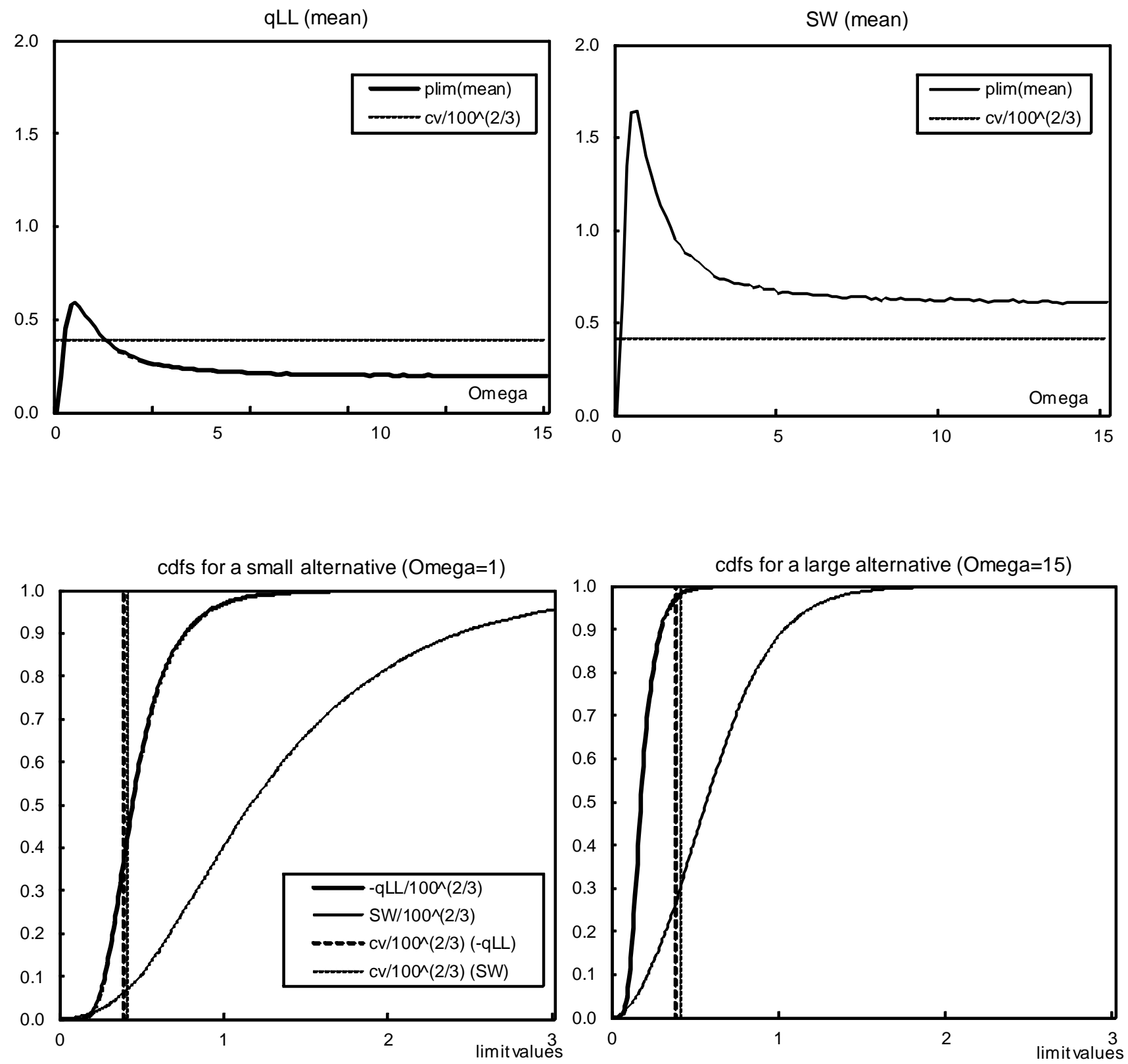
Figure 10a : Limits of $-T^{-1} q \hat{L} L$ and $T^{-1} S W$, dynamic regression

Random level shift model with intensity $p / T=0.05$
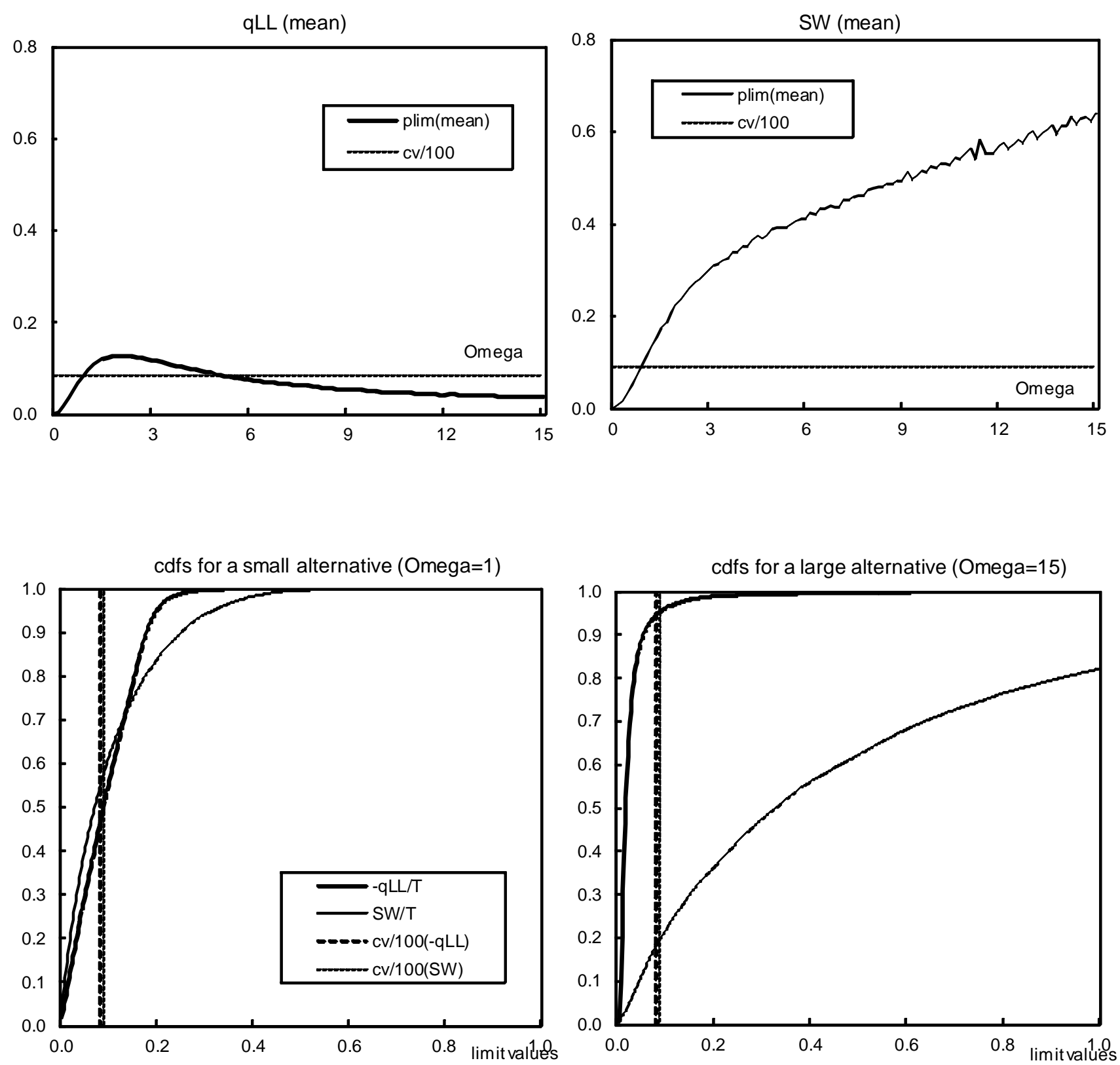
Figure 10b : Limits of $-T^{-1} q \hat{L} L$ and $T^{-1} S W$, dynamic regression

Random level shift model with intensity $p / T=0.1$
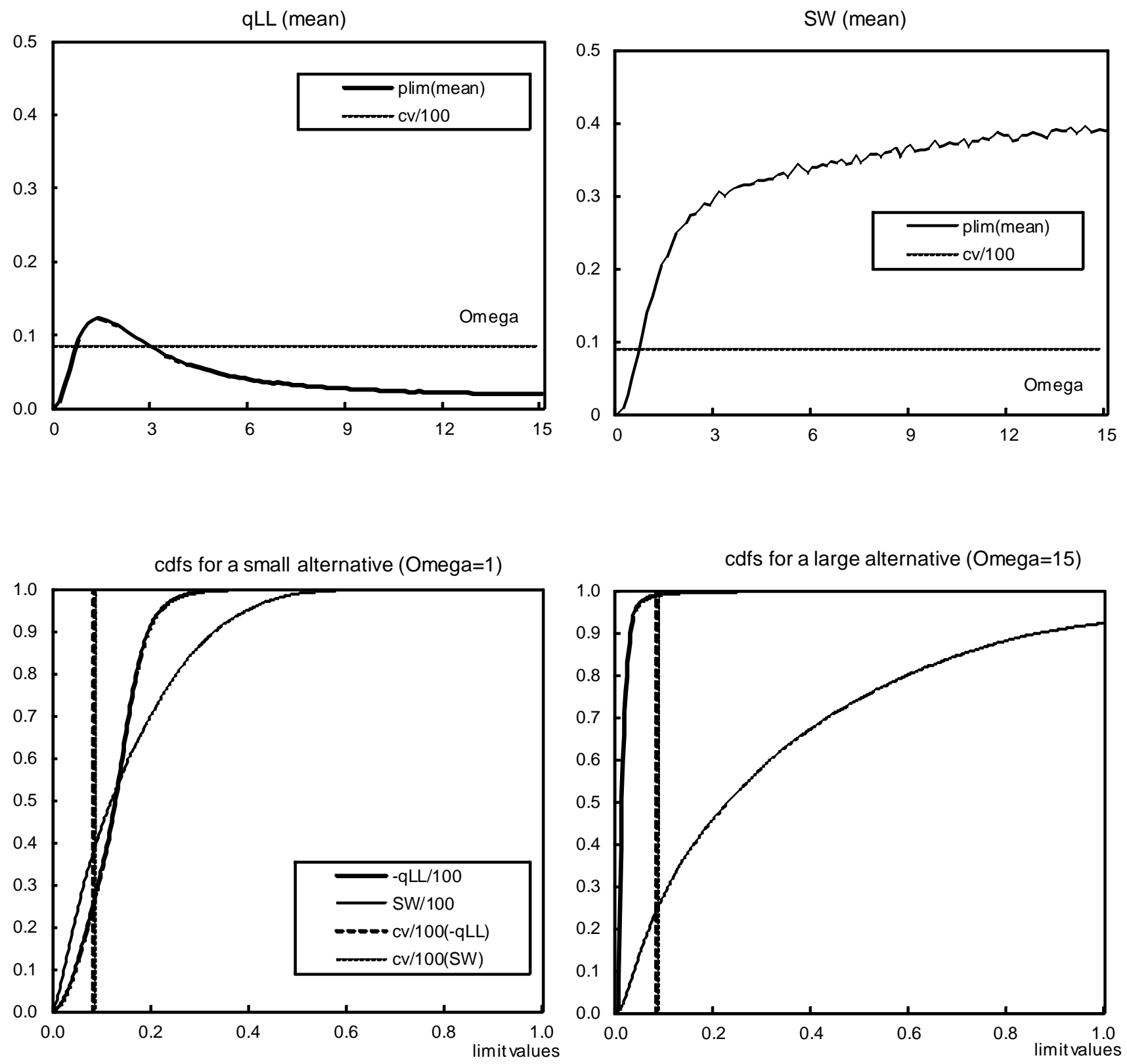
Figure 10c : Limits of $-T^{-1} q \hat{L} L$ and $T^{-1} S W$, dynamic regression

Random level shift model with intensity $p / T=0.5$
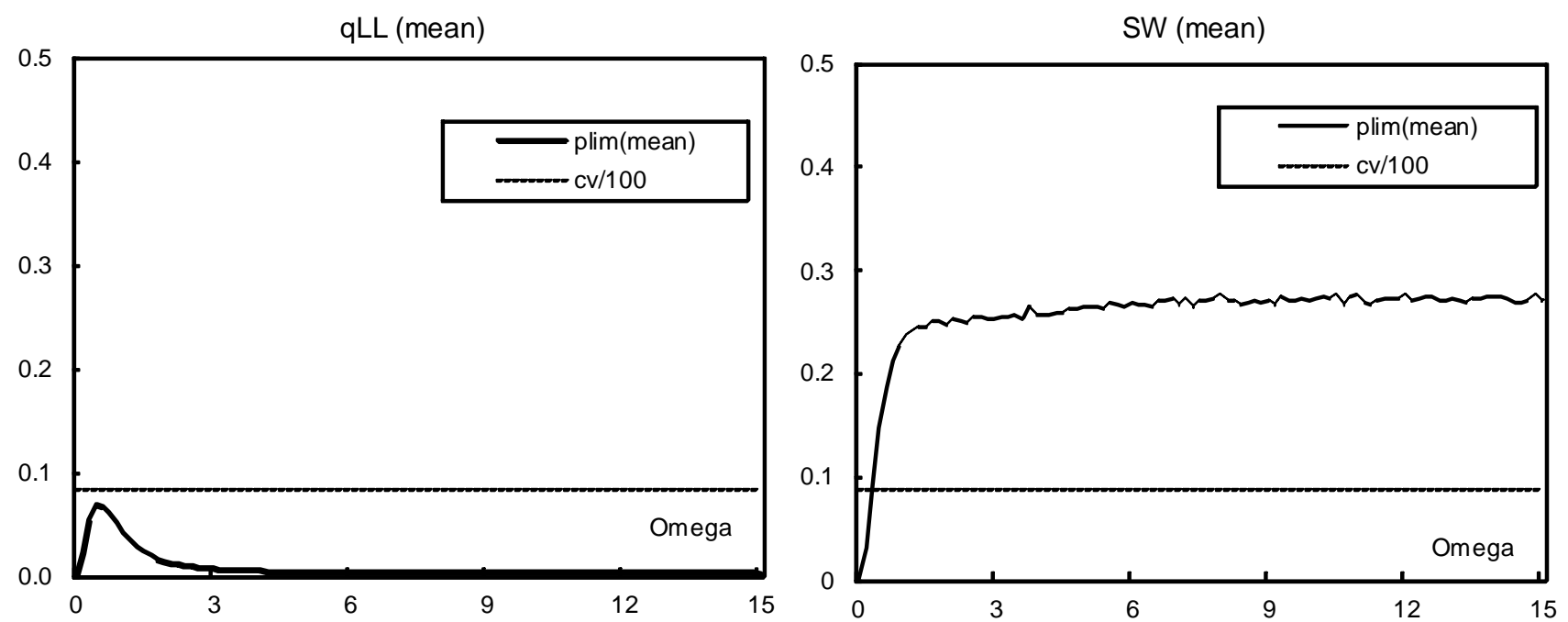

cdfs for a small alternative (Omega $=1)$

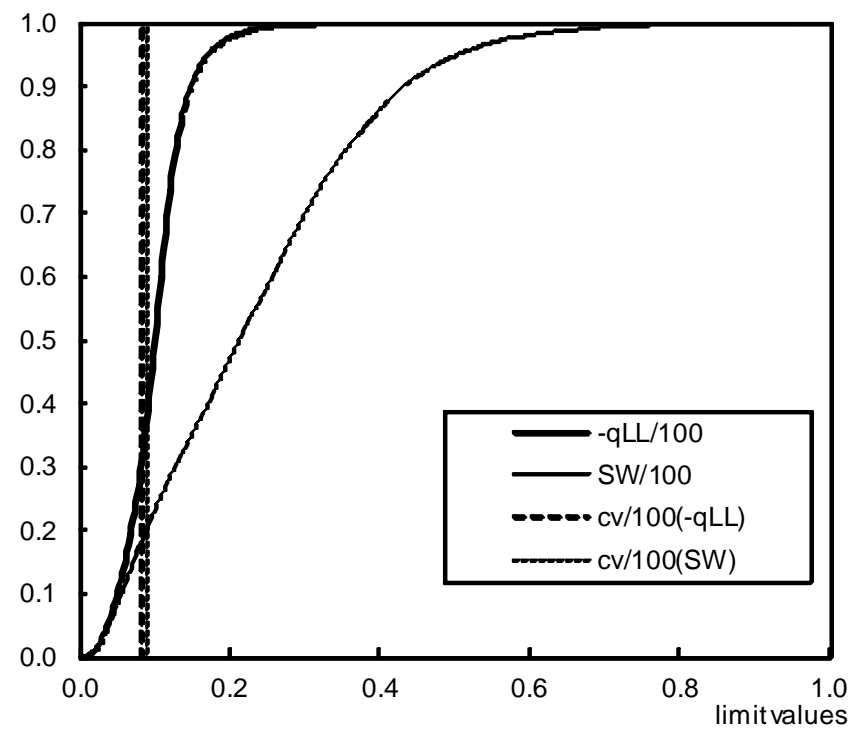

cdfs for a large alternative (Omega=15)

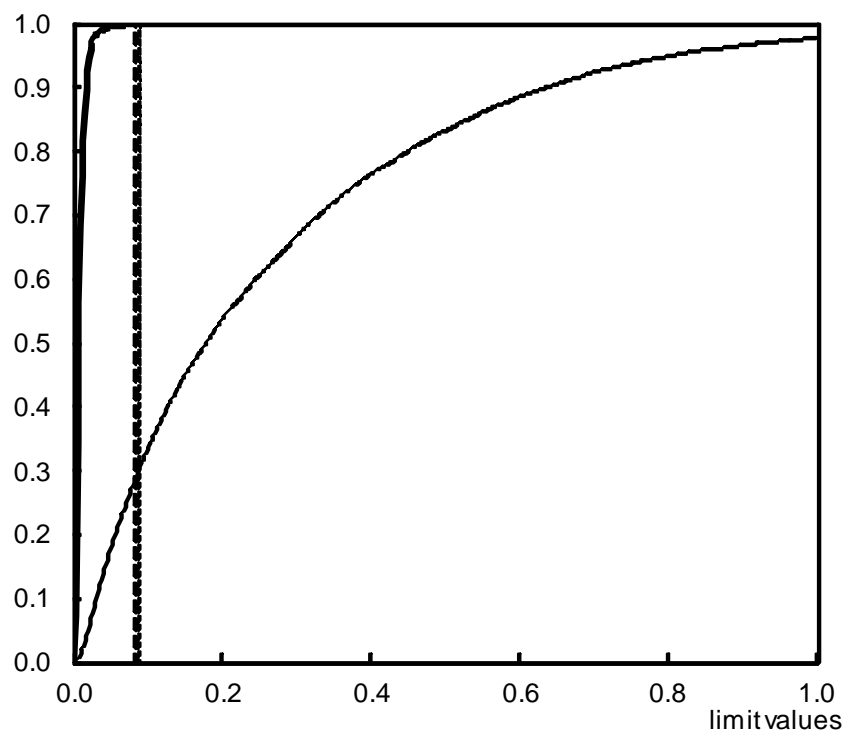


Figure 11: Limits of $-T^{-1} q \hat{L} L$ and $T^{-1} S W$, non-robust test

Single fixed break model
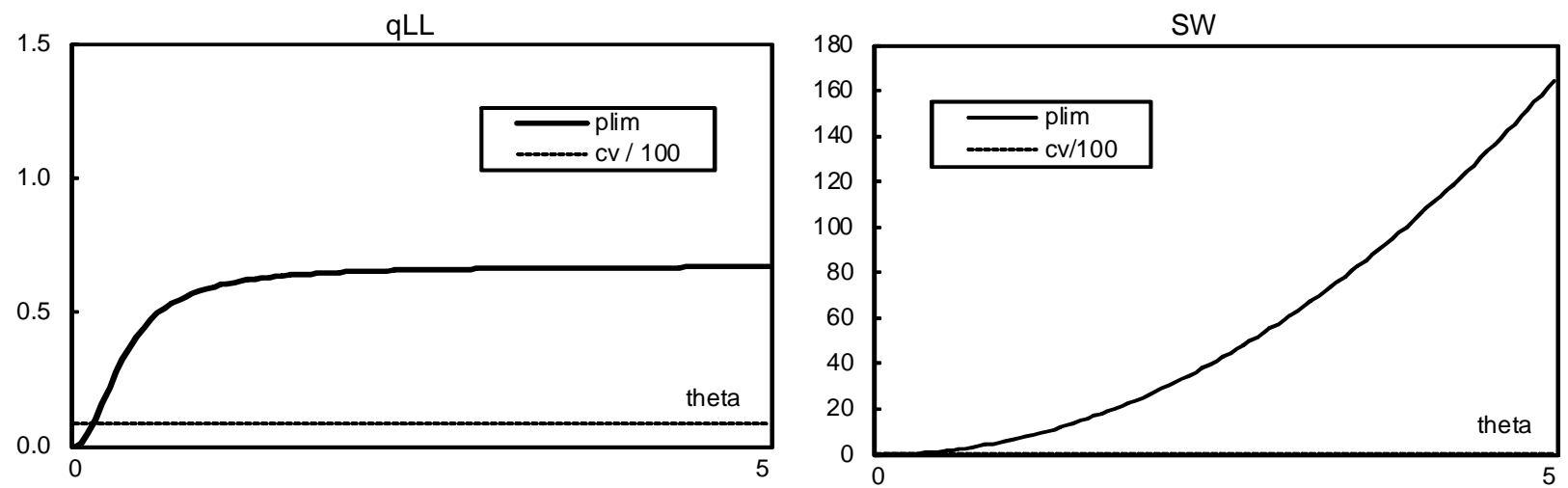

Figure 12: Limits of $-T^{-2 / 3} q \hat{L} L$ and $T^{-2 / 3} S W$, HAC robust test

Single fixed break model
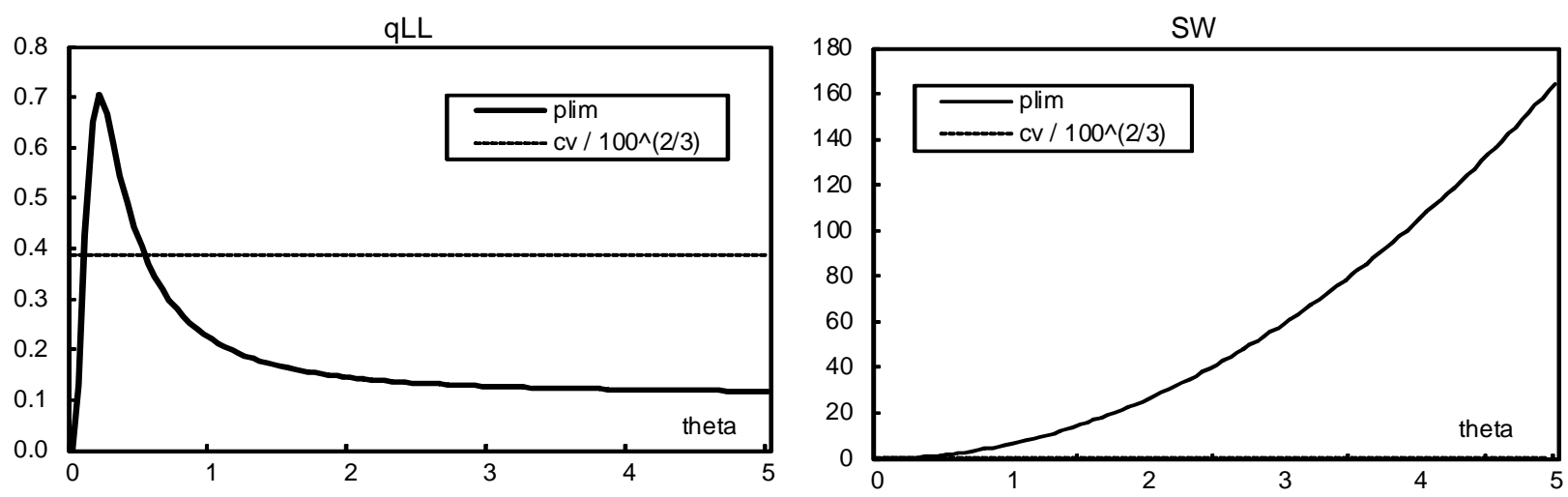

Figure 13. Limits of $-T^{-1} q \hat{L} L$ and $T^{-1} S W$, dynamic regression

Single fixed break model
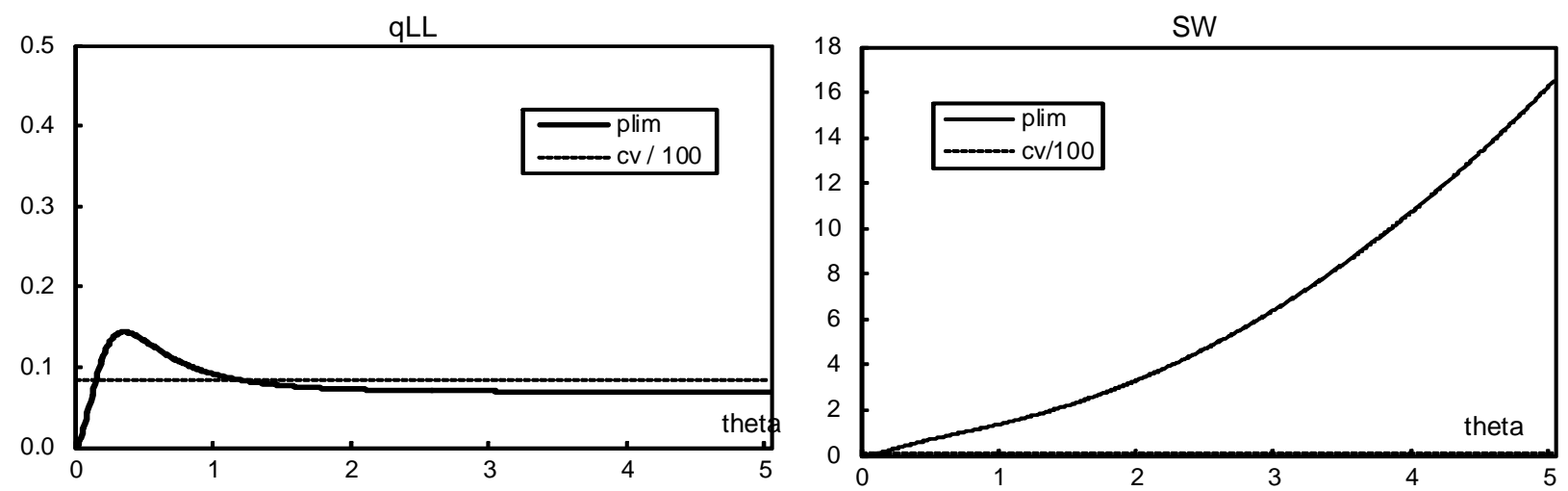